\title{
A numerical performance analysis of a ducted, high-solidity tidal turbine
}

\author{
Mitchell G. Borg ${ }^{\mathrm{a}}$, Qing Xiao ${ }^{\mathrm{a}, *}$, Steven Allsop ${ }^{\mathrm{b}}$, Atilla Incecik ${ }^{\mathrm{a}}$, Christophe Peyrard ${ }^{\mathrm{c}}$ \\ ${ }^{a}$ Department of Naval Architecture, Ocean, and Marine Engineering \\ University of Strathclyde \\ Glasgow, Scotland, United Kingdom \\ ${ }^{b}$ Industrial Doctoral Centre for Offshore Renewable Energy (IDCORE) \\ University of Edinburgh \\ Edinburgh, Scotland, United Kingdom \\ ${ }^{c}$ Electricité de France Research and Development \\ EDF R\&D \\ Chatou, Ile-de-France, France
}

\begin{abstract}
This study puts forward an investigation into the hydrodynamic performance concerning a ducted high-solidity tidal turbine utilising blade-resolved computational fluid dynamics. The model achieves similarity values of over 0.96 with experimentation data regarding a three-bladed horizontal-axis tidal turbine in validation of three distinct parameters: power \& torque coefficient, thrust coefficient, and wake velocity profiles. Accordingly, the model was employed for the analysis of a ducted, high-solidity turbine in axially-aligned flows at distinct free-stream velocities. The resultant hydrodynamic performance characteristics portrayed a peak power coefficient of 0.34 , with a thrust coefficient of 1.00 , at a nominal tipspeed ratio of 1.75. Coefficient trend agreement was attained between the numerical model and experimentation data established in literature and blade-element momentum theory; the model furthers the analysis by elaborating the temporal hydrodynamic features induced by the fluid-structure interaction in specification to the wake formation velocity profiles, pressure distribution along the blades and duct, volumetric flow rate, and vortex shedding effects to establish the characteristic flow physics of the tidal turbine.

Keywords: turbine performance, high solidity, open centre, tidal turbine, ducted turbine, RSM
\end{abstract}

\section{Introduction}

Efforts to increase the efficiency of energy-generating turbines have been in constant development following their implementation in the global market. At the forefront of the

${ }^{*}$ Corresponding author

Email addresses: mitchell.borg@strath.ac.uk (Mitchell G. Borg), qing.xiao@strath.ac.uk (Qing Xiao), christophe.peyrard@edf.fr (Christophe Peyrard) 
pertinent research is the effort of increasing the mass flow through the turbine, along with the constrainment and alignment of the wake flow, to facilitate further turbine installations [1]. As a result of the research and development attained, two conventional classifications of installations have been implemented around a turbine rotor: unidirectional ducts and bidirectional ducts. Both systems have been considered to enhance the performance of a turbine by increasing the flow velocity along the structure as a result of the induced physical phenomena. Albeit the potential improvements, the performance capabilities of a rotor within a bidirectional duct are inadequately understood.

The physics governing the flow within a vacant duct, in contrast, are widely understood. In accordance to Bernoulli's principle, the energy state of an ideal fluid is a relationship between its kinetic and potential energy, hence a function of its pressure, density, and velocity, through a spatial discrepancy [2]. In consideration of a bidirectional duct with a constricted section, hence a decrease in internal cross-sectional area, a Venturi effect phenomenon is induced through the system. Through this effect, in concordance to the principle of mass continuity, the axial velocity of an incompressible fluid increases along the constriction, along with a decrease in static pressure. In this confine, the application is numerically simplistic if a finite section of a duct system is considered, as the parameters are largely consistent. Nonetheless, an open-ended duct succumb to variable parameters of an external fluid domain, together with the implementation of a rotor, which, as a structure, will vary the pressure and velocity gradients through the duct, will induce high degrees of complexity. The potential increase in flow velocity through the turbine, however, as a result of the Venturi effect, is of particular interest. For this reason, the aim of this research is to analyse the hydrodynamic performance of a ducted high-solidity tidal turbine, particularly within the presence of duct-rotor influence.

Albeit a handful of recent research ventures have tackled the fluid dynamics of the implementation of a duct feature in a turbine system, they have had numerical forms of methodological limitations, such as analytically capturing mass and momentum continuity through the duct [3], or discretising the blade structure into elements [4,5], rather than portraying its true physicality. This research strives to overcome the related limitations by implementing a computational fluid dynamic (CFD) analysis with blade-explicit actuality, coupled with general continuity at both the domain and turbine. This feature allows the aspect of modelling flow three-dimensionality at the turbine, together with induced turbulence effects, in further effort of numerically replicating its true performance. Validated by means of a direct comparison to experimentation in numerically replicating the physics of rotating blades, the numerical outcomes of the main performance factors of the full-scale ducted turbine, in terms of power, torque, and thrust coefficients, were compared to literature [6] and blade-element momentum theory [4] to ascertain the applicability of the full-scale analysis, and justify further fluid dynamic analysis, namely the hydrodynamic effects along the duct, mass-flow rate through the duct, vortex shedding within the domain, static pressure along the rotor blades, and velocity profiles within the rotor wake. This written work is composed of six sections in discourse of: (i) an introduction to the concept of ducted turbines, with an overview of its state-of-the-art, (ii) the methodologies utilised for its numerical analysis, (iii) a characterisation of turbine theory and the modelling schemes utilised, (iv) a confirmation 
of the numerical model validation, (v) a discussion of the results attained, together with (vi) a general conclusion of the analysis.

\subsection{Commercial Application}

A number of commercial endeavours have attempted to adopt ducted turbine technology in achieving economical prospects, yet a substantial quantity of the investments have ensued in insolvency and cessation [7]. Amongst the ventures, OpenHydro Ltd. had, disputably, attained quasi-sustainable implementation with an open-centre ducted design approach in which an aperture is employed at the centre of the turbine in replacement of a turbine nose cone hub $[8,9]$. Due to this approach, the setup not only utilises the duct as a flow modifier, but also as a unit in which the power generating system may be housed.

Tidal stream turbine (TST) technology has been in its primal, developmental phase for the past decade with substantial potential, yet has not taken off as rapidly as had been predicted. This outcome has been argued to be largely due to engineering challenges in designing for extreme operating conditions, in combination with political and environmental factors, which has limited the rate of industry maturity [1]. Amongst the earliest groundbreaking tidal projects, the MCT SeaGen, a 1.2 MW twin-rotor device, was installed within the Strangford Loch, Northern Ireland in 2008, and, subsequent to generating $10 \mathrm{GWh}$ of electrical supply, decommissioned in 2016 [10]. Yet, with very few corresponding projects attaining a similar degree of impact in the renewable energy field, corporate interest has largely diminished within the tidal industry. Albeit the drawbacks observed, European, North American, and Asian nations and unions have persisted their investment in the deployment of full-scale tidal turbine arrays, with a wide range of the European investment being installed within territorial waters of France and the United Kingdom.

A profusion of turbine designs have undergone hydrodynamic investigations for tidal applications, yet two configurations have been largely integrated within the industry, both of which having attained throughput to commercialisation deployment scales. Characteristic to the aerodynamic equivalent, three-bladed horizontal axis tidal turbine (HATT) designs have been commissioned in large-scale installations. Having successfully installed four HATTs in its primal stages, Phase 1A, the MeyGen project is amongst the more notable commercial efforts in HATT energy generation. Future prospects include Phases $1 \mathrm{~B}$ and $1 \mathrm{C}$, potentially introducing an additional $6 \mathrm{MW}$ (four turbines) and 73.5 MW (forty-nine turbines) respectively to the grid, together with Phases 2 and 3 currently within planning stages. In continuation, the ducted turbine configuration has enticed interest due to the potential increase in power extraction as a result of the enhancement of mass flow through the rotor, in addition to benefits as: alignment of axial velocity in yawed flows, providing housing for direct-drive rim generators, and elimination of subsidiary mechanical systems and components, such as gearboxes. DCNS/OpenHydro Ltd. had successfully installed a $2 \mathrm{MW}$ turbine in the Bay of Fundy, Canada, portrayed in Figure 1a, together with a pair of 500 $\mathrm{kW}$ rated capacity turbines, illustrated in Figure 1b, as a demonstration array in PaimpolBréhat, Northern France, in collaboration with EDF France. Yet, despite the milestones, the imminent economic instability of the development of ducted turbines may potentially falter the perception of this technology. 


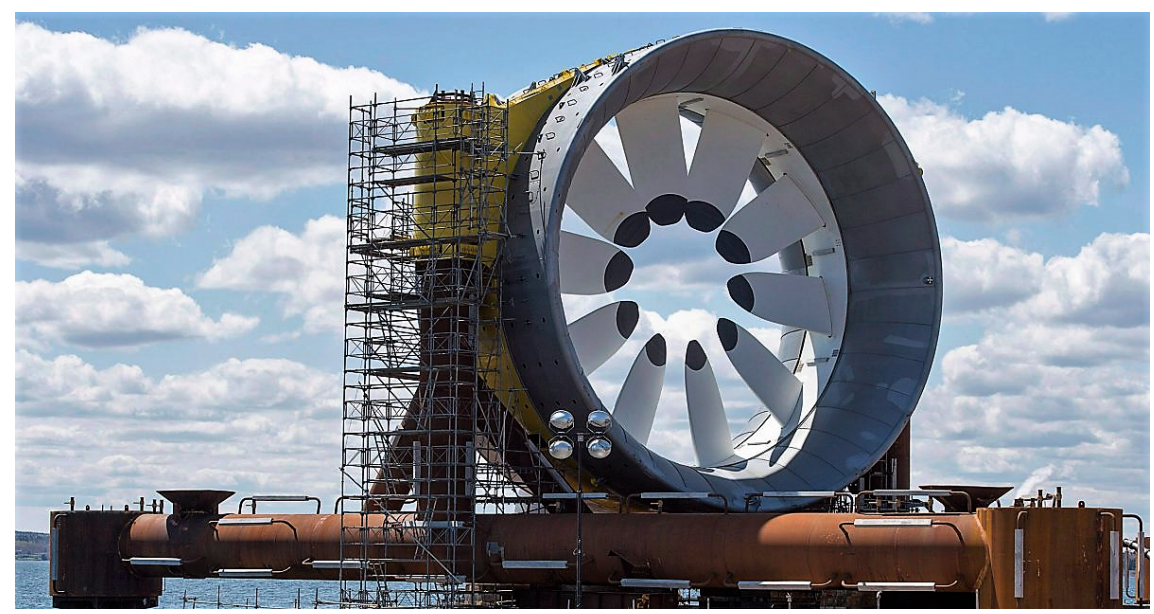

(a) 2 MW-rated turbine for the Cape Sharp project [11]

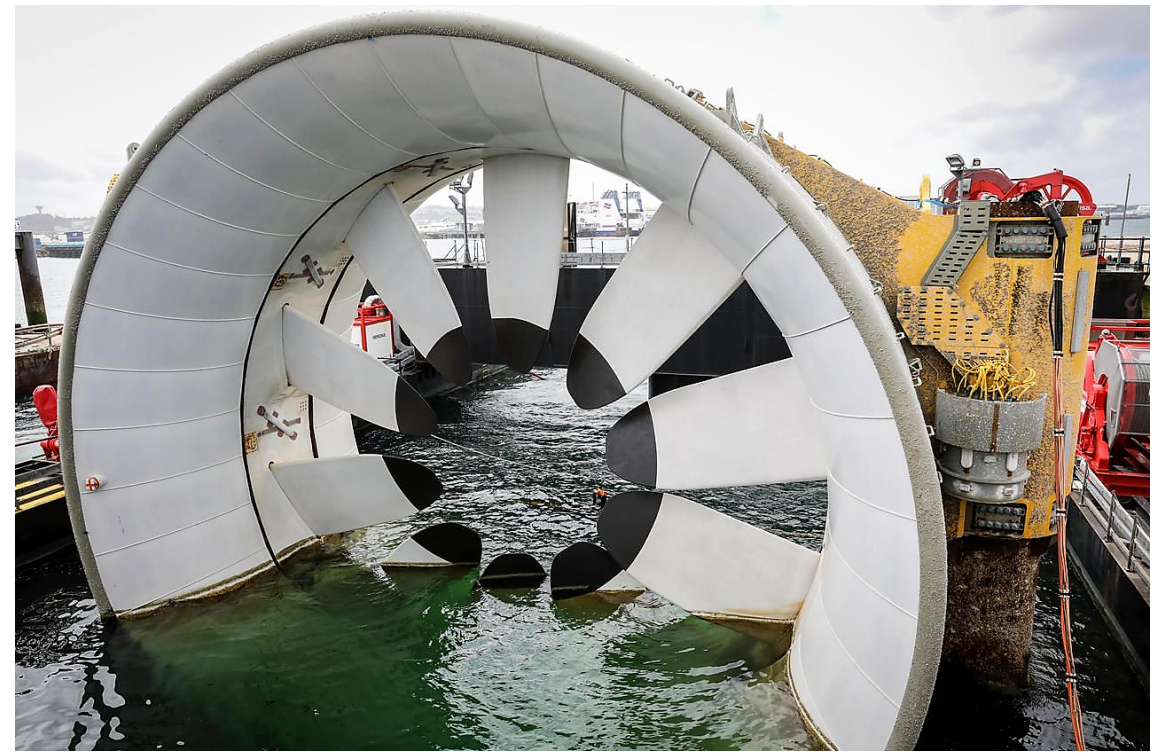

(b) $500 \mathrm{~kW}$-rated turbine at the Paimpol Bréhal site in Northern France [12]

Figure 1: The OpenHydro PS2 Ducted Turbine

\subsection{State of the Art}

Investigative analyses regarding the efficacy of ducted turbines have been mainly directed by numerical methodologies. Primarily instigated by wind turbine analyses, Phillips et al. [13, 14] developed a diffuser-type (or unidirectional) duct for the now-inactive Vortec Energy. Having undertaken both experimentation and CFD analyses for the turbine, good agreement was attained for velocity increases and pressure through the duct, yet power outcomes were found to be overestimated in modelling results, largely due to the implementation of the an actuator disc model together with the incapacity of the Standard $k-\varepsilon$ turbulence model in capturing certain separation effects. The effect of the disc loading was studied, which acknowledged that a large degree of intermittent flow separation occurred at 
the trailing third of the outer duct region in both experimentation and modelling. At high disc loading, where the outlet flow acts at a high velocity, however, it was noted that the separated region on the diffuser had been reduced.

Visser [15] analysed a ducted wind turbine design in test fields implementing a boundary layer control slot for pressure distribution within its diffuser section, whereas Hansen et al. [16] numerically established a CFD model of a diffuser by implementing an actuator disc in representation of a wind turbine. Werle and Presz [17] presented a theoretical basis, with numerical validation, of the potential improvements available from ducted flow configurations. Grassmann et al. [18, 19] analysed a diffuser consisting of two aerofoil sections utilising a similar segregated control slot by means of both experimentation and numerical simulation; Ohya et al. [20] tested a flanged diffuser to establish an optimal duct shape.

Shifting to their implementation in tidal turbine applications, Munch et al. [21] modelled a three-dimensional CFD simulation of a ducted turbine by means of a moving mesh methodology with blade actuality, albeit in a unidirectional aerofoil-shaped diffuser setting. In an effort of optimising both rotor and duct geometry, a nominal rotor power coefficient of 0.55 was attained for the four-bladed system. Gaden and Bibeau [22] investigated differing diffuser geometrical parameters by utilising an actuator disc representation region to extract axial momentum. Shives and Crawford [23] analysed a ducted CFD actuator disc model by varying the angle of attack of the unidirectional duct profile. In comparing two turbulence methodologies, RANS and LES, in low-wave conditions, Ahmed et al. [24] acknowledged the inaptness of resolving the wake turbulence by means of RANS turbulence modelling. Holst et al. [25] conducted a numerical study to analyse the wave-induced loads on tidal turbine blades at distinct angles of attack. This was then compared to BEM analyses, which depicted similar parameter outcomes.

In correspondence to the natural properties of tides, tidal turbine ducts have been industrially considered within a bidirectional setup. Setoguchi et al. [26] conducted an experimentational study utilising vacant ducts to investigate the most efficient shape for a two-way diffuser in a fluid-flow energy conversion system. Fleming and Willden [27] investigated the difference in velocity through the throat of a bidirectional duct in accordance to distinctive duct profiles, where the implemented turbine was represented as an actuator disc utilising CFD. In continuation, Allsop et al. [3, 4] developed a numerical blade-element momentum theory (BEMT) model to analyse flow through a duct in interaction with a high-solidity embedded rotor. Discretising the blade structure into elements, rather than portraying its entirety, the methodology analyses the turbine hydrodynamic performance by coupling the performance of the individual element sections, where the forces acting on the elements are then analysed utilising actuator disc/momentum theory coupled with the Bernoulli principle, to replicate the Venturi effect, with empirical corrections for duct interaction. Belloni et al. [5, 28, 29] investigated the effects of different bidirectional duct setups utilising a coupled BEM-CFD method to improve the capturing of mass and momentum continuity through the duct, avoiding the analytical limitations of the Bernoulli principle, together with representing turbulence effects.

The numerical analysis elaborated in this present study is a continuation of Borg et al. [30], which develops a real-scale CFD model to assess the hydrodynamic performance 
of a high-solidity open-centre tidal turbine within a bidirectional duct. This investigation evaluates the hydrodynamic effects of the duct on the turbine, where the individual turbine blades, rather than an area-averaged disc, are established, hence inferring the outcomes in quasi-real-ocean conditions. The hydrofoil geometry was provided by EDF R\&D to replicate the outcomes of a turbine similar to the design of the OpenHydro PS2 device. In addition, the 7-equation Wilcox Reynolds Stress 'Stress-Omega' Model (Stress- $\omega$ ) turbulence model was coupled to close the Navier-Stokes equation and analyse the anisotropic flow domain [31].

\section{Numerical Methodology}

In description of the procedure with which the hydrodynamic analysis was tackled, the turbine setup and flow parameters of the analysis are elaborated within the framework of the computational fluid dynamic solver numerical setup.

\subsection{Turbine Setup}

In an effort to attain a validated CFD model for tidal turbine applications, simulations were established to numerically replicate experimentation undertaken by Mycek et al. [32]. Identical blade, nacelle, and mast geometry were utilised within the model domain, illustrated in Figure 2, onto which a tetrahedral mesh was imprinted. The general dimensions of the turbine include a diameter $D$ of $0.7 \mathrm{~m}$, a nacelle length of $2.5 \mathrm{~m}$, and a mast length of $1 \mathrm{~m}$; supplementary descriptions may be attained within the literature. A distinction is present, however, between the physical aspects of the experimentation and the numerical model. In the prior, the nacelle mast protrudes through the free-surface to connect to the above support, whereas, in the latter, only the submerged length of the mast is considered, with a hemisphere at the tip to avoid tip-induced vortices. Hence, a physical assumption is present within which the drag forces on the mast are solely considered to be due to the current, rather than any additional wave \& wind factors. Albeit, due to the fact that waves were not induced in the experimentation, and that the setup was in a closed environment, the assumption that free-surface and topside effects were insignificant when considering the induced physics of the turbine was held. The parameters of the turbine and fluid flow were also instated from the literature, with a constant free-stream velocity of $0.8 \mathrm{~m} / \mathrm{s}$, a tipspeed ratio (TSR) range of $1.00-8.26$, an inlet turbulent length scale of $1 \mathrm{~m}$, and an inlet turbulence intensity of $3 \%$.

In continuation, upon validation, the model settings were implemented for the analysis of a ducted eight-bladed tidal turbine, similar to the design of the OpenHydro PS2 device. The general dimensions of the turbine were provided by EDF R\&D, which describe a rotor diameter of $12 \mathrm{~m}$, a duct diameter of $15 \mathrm{~m}$, and a duct length of $10 \mathrm{~m}$, as illustrated in Figure 3; supplementary descriptions are available in Table 1. The hydrofoil sections comprising the blades consisted of a flat-plate design with rounded edges. The geometry is quasi-identical to Allsop et al. [3], being slightly adapted to attain a more homogeneous blade surface, as described in Table 2. The turbine geometry was implemented within the model domain, onto which a tetrahedral mesh was imprinted. In resemblance to the validation procedure, the physical model, was designed to consist of identical domain layout 
and boundary conditions as the three-bladed HATT numerical model. The parameters of the turbine and fluid flow were instated from real-world data, recommended by EDF R\&D, as reported in Neill et al. [33] and Bahaj and Myers [34], with maximum acquired spring tide velocities of $4.0 \mathrm{~m} / \mathrm{s}$, and surface velocities of $5.0 \mathrm{~m} / \mathrm{s}$, respectively. For this reason, the free-stream velocity range initiated varied between $1 \mathrm{~m} / \mathrm{s}-7 \mathrm{~m} / \mathrm{s}$, in iterations of 1.5 $\mathrm{m} / \mathrm{s}$, with an inlet turbulence intensity of $3 \%$ and an inlet turbulent length scale of $1 \mathrm{~m}$, to acquire a definitive performance spectrum of the turbine. Within this free-stream velocity variation, a turbine TSR range of $1.00-2.50$ was considered as the nominal region, both due to high-solidity predictions specified by Betz [6], and analysis by Allsop et al. [4].

\subsection{Numerical Setup}

Inferred as fully submerged systems within a constant fluid flow, the models were founded under the premise that no far-stream effects are in interaction with the turbines, hence unaffected by the implications of the free-stream and the seabed; this was purposely implemented to acknowledge the performance of the turbines under ideal, consistent temporal conditions.

Implementing the use of the commercial solver ANSYS Fluent 16.1, the CFD physical models were designed to consist of a cuboidal external domain layout, imposed with relevant boundary conditions. The CFD solver was utilised in computing time-averaged representations of the continuity and Navier-Stokes equations, which govern the three-dimensional,

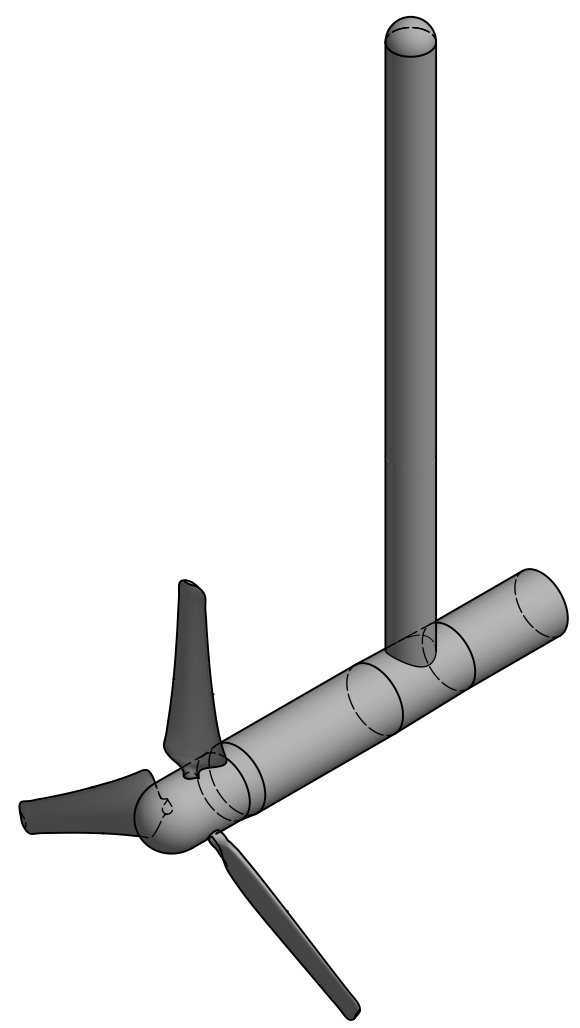

Figure 2: Rendered three-dimensional CAD representation of the horizontal-axis tidal turbine [32] utilised for the validation of the CFD model 


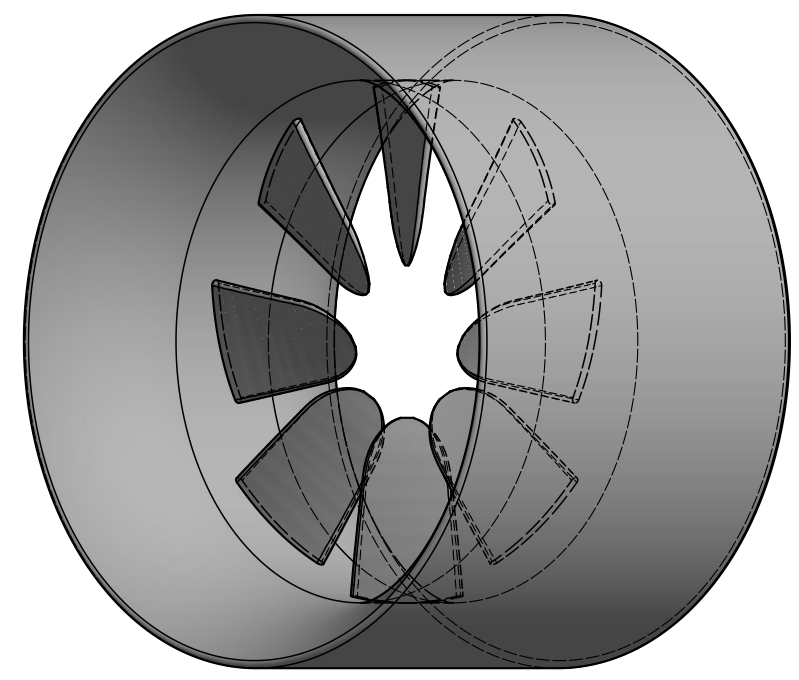

(a) Rendered three-dimensional CAD representation
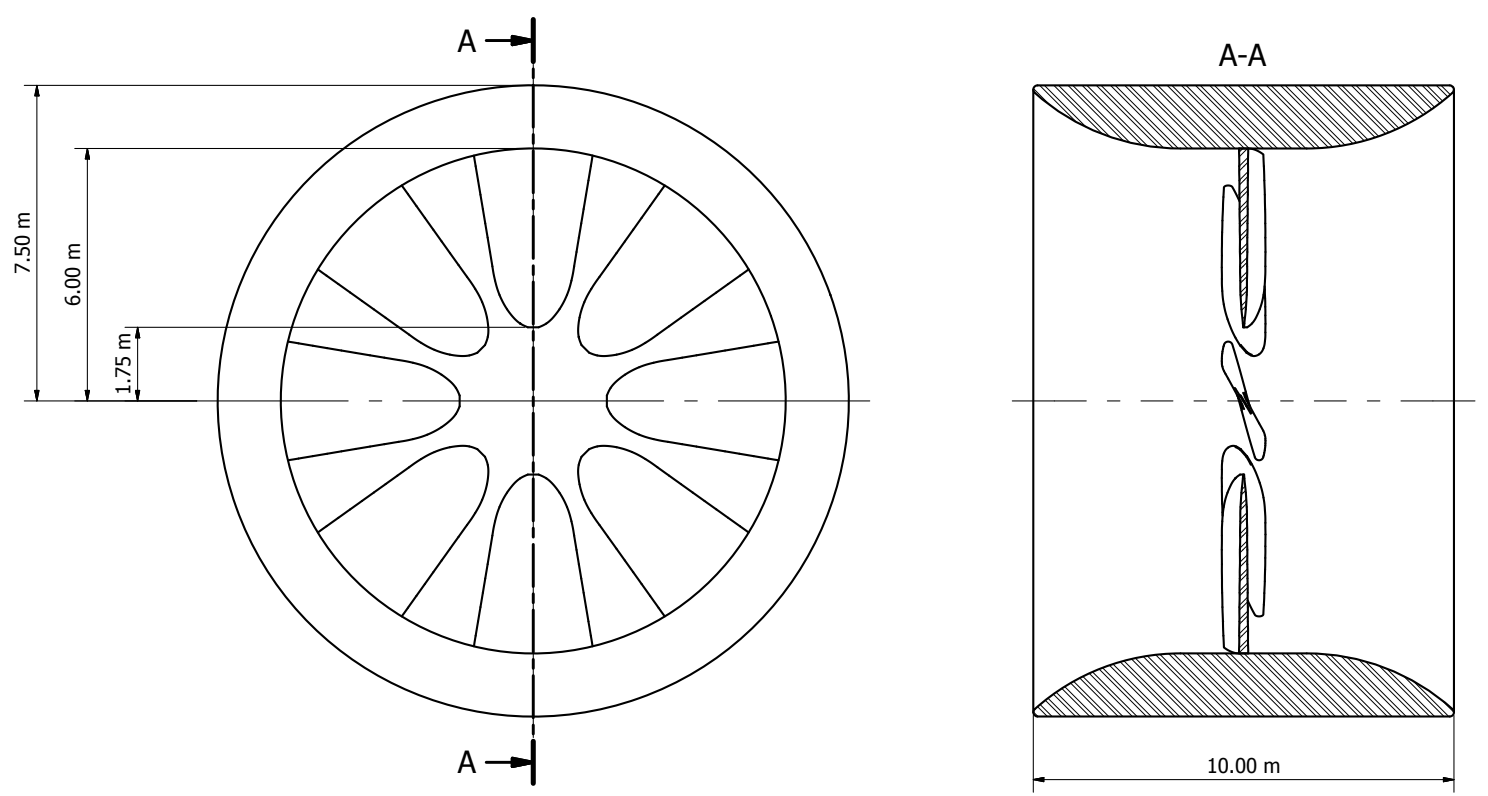

(b) First-angle sectioned projection

Figure 3: Geometrical model of the ducted high-solidity tidal turbine utilised for the CFD analyses

unsteady, incompressible fluid flow, along with a Reynolds-Averaged Navier-Stokes (RANS) turbulence model utilised in mathematical closure to represent flow property fluctuation. For both turbine models, the domain surrounding the turbine rotor was segregated from the global domain in an effort to induce a moving-mesh model with rotation at the turbine rotor relative to a stationary outer domain, with interfaces between the two domains. No-slip wall conditions were instigated on the rotor, mast and nacelle, and duct structures, whereas slip 


\begin{tabular}{ccc}
\hline Description & Validation Turbine [32] & Ducted Turbine \\
\hline \hline Blade Profile & NACA 63418 & Round-Edged Flat Plate \\
Rotor Radius $\left(R_{r t r}\right)$ & $0.35 \mathrm{~m}$ & $6.00 \mathrm{~m}$ \\
Hub Radius $\left(R_{h u b}\right)$ & $0.046 \mathrm{~m}$ & $1.75 \mathrm{~m}$ \\
Duct Radius $\left(R_{d c t}\right)$ & $\mathrm{N} / \mathrm{A}$ & $7.50 \mathrm{~m}$ \\
Hub Length $\left(L_{h u b}\right)$ & $0.72 \mathrm{~m}$ & $\mathrm{~N} / \mathrm{A}$ \\
Duct Length $\left(L_{d c t}\right)$ & $\mathrm{N} / \mathrm{A}$ & $10.00 \mathrm{~m}$ \\
Linear Velocity $\left(U_{\infty}\right)$ & $0.8 \mathrm{~m} / \mathrm{s}$ & $1-7 \mathrm{~m} / \mathrm{s}$ \\
Tip-Speed Ratio $(T S R)$ & $1.00-8.26$ & $1.00-2.50$ \\
Rotor Solidity $\left(S_{r t r}\right)$ & 0.132 & 0.614 \\
Rotational Orientation & counter-clockwise & $\mathrm{clockwise}$ \\
Reynolds Number $\left(R e_{\infty}\right)$ & $0.279 \cdot 10^{6}$ & {$[5.97-41.80] \cdot 10^{6}$} \\
Reynolds Number $\left(R e_{L}\right)$ & $0.573 \cdot 10^{6}$ & {$[9.95-69.67] \cdot 10^{6}$} \\
Reynolds Number $\left(R e_{c h}\right)$ & {$[0.049-0.245] \cdot 10^{6}$} & {$[3.15-37.49] \cdot 10^{6}$} \\
\hline
\end{tabular}

Table 1: Summary of Turbine Model Descriptions

conditions were instigated on the boundaries of the domain.

In a similar layout employed by Mason-Jones et al. [14], the dimensions of the computational domain were contrived to attain a numerical blockage ratio $\left(\alpha_{b l}\right)$ of less than $2 \%$ to the turbine to ascertain the absence of far-field effects, replicating real-ocean conditions. This corresponded to a cross-sectional domain dimension of $7 D$ along the edges of the quadratic face, with the rotor cylindrical domain positioned in the centre of the vertical plane. In consideration of the wake analysis, a domain length of $9 D$ was instituted, where the inlet and outlet planes were situated at a distance $3 D$ upstream and $6 D$ downstream of the turbine, respectively, as illustrated in Figure 4a. The time-step considered was designated in relation to the tip-speed ratio of the rotor, where each transient iteration is temporally equivalent to one-half of a degree of a turbine revolution, therefore attained by:

$$
\Delta t_{\text {step }}=\frac{1}{2} \times \frac{2 \pi}{360 \times \Omega_{\text {sys }}}
$$

where $\Delta t_{\text {step }}$ is the time-step and $\Omega_{\text {sys }}$ is the rotational velocity of the system. Having undertaken a time-step independence analysis, this was found to suffice both in sustaining stability in the model, and in achieving a sufficient Courant number at regions of interest throughout the domain.

Turbulence was modelled by means of the Reynolds-Stress Model (RSM) 'Stress-Omega' turbulence model. This model was opted for three distinctive reasons: i) as a variable, the specific dissipation rate $(\omega)$ is superior to the dissipation rate $(\varepsilon)$ in establishing the turbulent length scale as a result of its capacity in modelling all degrees of production, redistribution, and dissipation of turbulent kinetic energy within the flow, ii) mathematically, $\omega$ does not require the implementation of near-wall treatment equations to model the boundary layer at non-slip surfaces, as the function is able to be integrated to the wall, displaying greater 


\begin{tabular}{cccc}
\hline$r_{r t r} / R_{r t r}$ & $c_{r t r} / R_{r t r}$ & Pitch $\left(^{\circ}\right)$ & $t_{r t r} / c_{r t r}(\%)$ \\
\hline \hline 0.2917 & 0.0500 & 32.00 & 6.67 \\
0.3067 & 0.1208 & 31.66 & 4.14 \\
0.3350 & 0.1908 & 30.98 & 5.24 \\
0.3633 & 0.2383 & 30.30 & 6.29 \\
0.3917 & 0.2750 & 29.62 & 6.67 \\
0.4200 & 0.3025 & 28.94 & 7.16 \\
0.4483 & 0.3233 & 28.26 & 7.22 \\
0.4767 & 0.3383 & 27.58 & 6.90 \\
0.5050 & 0.3500 & 26.90 & 7.14 \\
0.5333 & 0.3583 & 26.22 & 6.98 \\
0.5617 & 0.3667 & 25.54 & 6.82 \\
0.5900 & 0.3750 & 24.86 & 7.11 \\
0.6183 & 0.3833 & 24.18 & 6.96 \\
0.6467 & 0.3917 & 23.50 & 6.81 \\
0.6750 & 0.4000 & 22.82 & 7.08 \\
0.7033 & 0.4083 & 22.14 & 6.94 \\
0.7317 & 0.4167 & 21.46 & 7.20 \\
0.7600 & 0.4250 & 20.78 & 7.06 \\
0.7883 & 0.4333 & 20.10 & 6.92 \\
0.8167 & 0.4417 & 19.42 & 7.17 \\
0.8450 & 0.4500 & 18.74 & 7.04 \\
0.8733 & 0.4583 & 18.06 & 6.91 \\
0.9017 & 0.4667 & 17.38 & 7.14 \\
0.9300 & 0.4750 & 16.70 & 7.02 \\
0.9583 & 0.4833 & 16.02 & 6.90 \\
0.9867 & 0.4917 & 15.34 & 7.12 \\
1.0000 & 0.5000 & 15.00 & 7.00 \\
& & &
\end{tabular}

Table 2: Detailed ducted turbine blade profile descriptions

accuracy within the log-linear range of the law-of-the-wall y-plus $\left(y^{+}\right)$spectrum, and iii) as a result of its non-implementation of the Boussinesq approximation, the model analyses all Reynolds stresses in three-dimensional space, prompting superiority in analysing anisotropic flows, such as flows over curved surfaces, flows in rotating fluids, and flows in ducts with secondary (rotational) motion [31]. Albeit the advantages of the Stress- $\omega$ turbulence model, specifically in wall-bounded flows, a significant drawback it presents is the potential oversensitivity in shear flow at high Reynolds numbers, similar to the Standard $k-\omega$ turbulence model, hence potentially portraying unphysically higher outcomes under exaggerated physics within the fluid-structure interaction phenomena.

In accompaniment to the Stress- $\omega$ model, the SIMPLE pressure-velocity coupling scheme setting, the Green-Gauss Node Based gradient, Pressure Staggering Option pressure, Sec- 


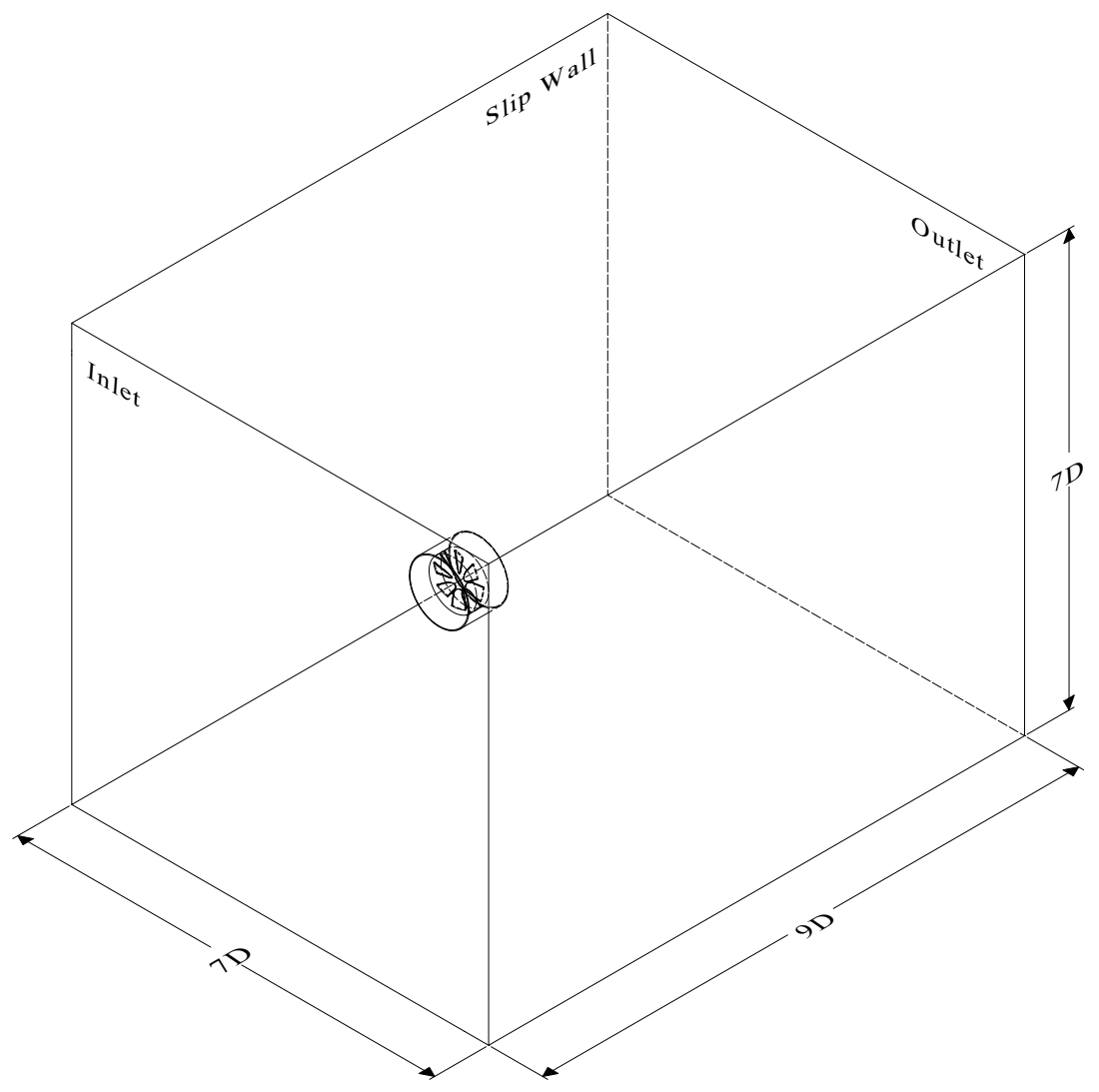

(a) Layout of the ducted turbine domain

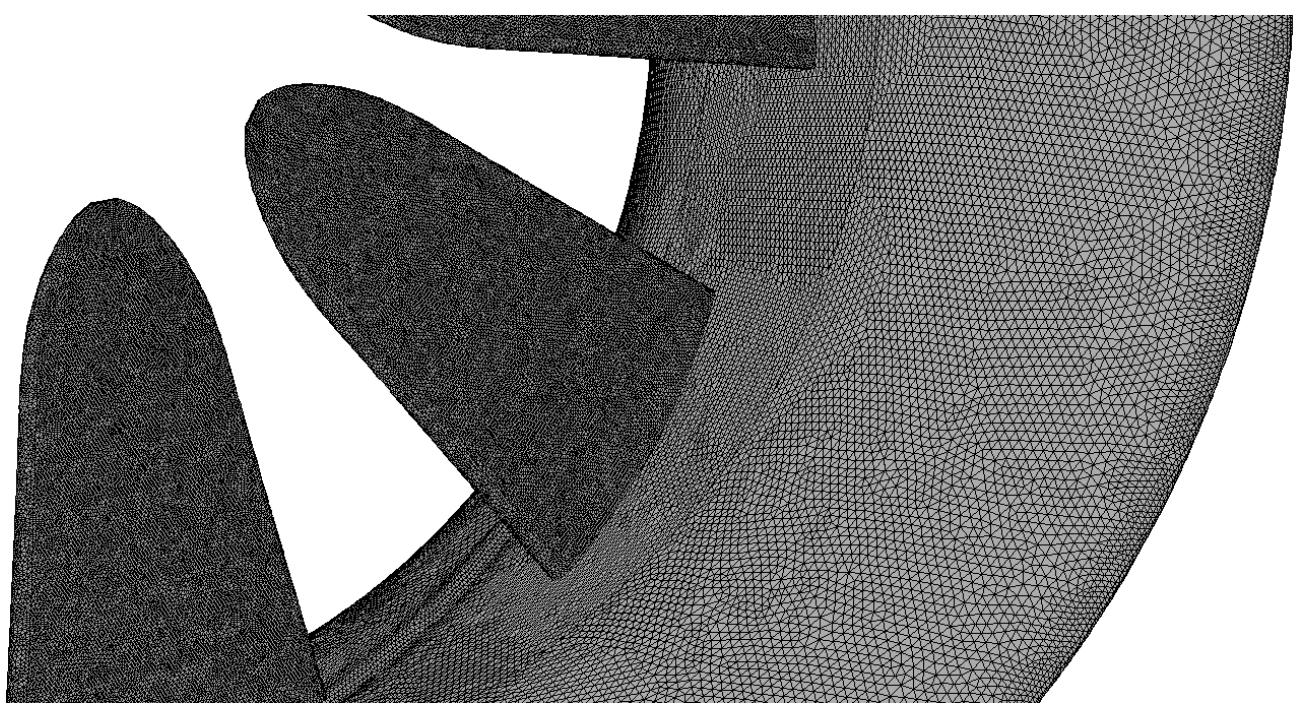

(b) Geometric tessellation of the mesh on the duct and rotor blades

Figure 4: Geometrical model of the ducted high-solidity tidal turbine utilised for the CFD analyses 
ond Order Upwind momentum, Second Order Upwind specific dissipation rate, and Second Order Upwind Reynolds stresses spatial discretisation settings, and the Bounded Second Order Implicit transient formulation setting were utilised as the solution methodologies. It should be stated that the dissipation rate was altered to Second Order from First Order settings once the resultant parameter outputs were steady in time, as initially running with the more accurate setting would result in the simulation to fail within the first few time-steps. Furthermore, the pressure setting was also altered from Second Order to Pressure Staggering Option once the resultant parameter outputs were steady in time; the prior setting greatly underestimates the parameters. In addition, for the successful use of the Stress- $\omega$ turbulence model with these settings, a quality mesh is required. Through empirical means, it was acknowledged that a mesh with skewness higher than 0.79 resulted in the failure of the simulation, highlighting the limited robustness of the turbulence model; other mesh qualimetrics had no such an effect.

A mesh independence procedure was primarily carried out on the three-bladed HATT by considering the parameter with the highest degree of dynamics; the highest TSR condition. Subsequent to the procedure, the final average surface mesh count utilised was marginally above 87,000 cell faces per blade, with dense volumetric cells around the turbine and within the wake, to achieve a total mesh count of 13.5 million for the three-bladed HATT, over 8 million of which within the turbine rotating region. An identical procedure was undertaken for the ducted turbine, attaining an average surface mesh of slightly above 94,000 cell faces per blade, illustrated in Figure 4b, with more than 18.5 million volumetric cells within the entire domain, over 10 million of which within the turbine rotating region. The mesh was implemented with a prism layer at non-slip surfaces with an appropriate cell height to achieve a y-plus value of $60 \leqslant y^{+} \leqslant 500$ across the blades and duct. This range was considered due to the high Reynolds number $\left(>10^{6}\right)$ of the system, hence modelling the viscous sublayer was abdicated to reduce computation time.

The CFD computations were performed using the ARCHIE-WeSt cluster facility at the University of Strathclyde by running two Intel Xeon Gold $61382.00 \mathrm{GHz}$ computational nodes, with 40 cores and $192 \mathrm{~GB}$ of RAM per node per simulation. A ducted turbine simulation was completed within roughly 75 wall-clock hours, equivalent to 3,000 core-hours, hence resulting in an average of 5 wall-clock hours per turbine rotation.

\section{Numerical Model Characterisation}

The characterisation of the numerical model, where the formulae utilised in relation to the physical aspects of the tidal turbine, and in analysis of the fluid continuum, are specified.

\subsection{Physical Modelling}

In consideration of the analysis of a physical turbine, notable definitions concerning the resultant performance outcomes, in terms of the boundary conditions employed, are identified. Utilised to attain quasi-real-ocean conditions, the blockage ratio $\left(\alpha_{b l}\right)$ is defined as a correlation between the device reference area $\left(A_{d v c}\right)$ and the domain sectional area 
$\left(A_{d m n}\right):$

$$
\alpha_{b l}=\frac{A_{d v c}}{A_{d m n}}=\frac{\pi R_{d v c}^{2}}{L_{d m n}^{2}}
$$

where $R_{d v c}$ is the device radius, and $L_{d m n}$ is the length of the quadratic cross-sectional area of the computational domain.

The tip-speed ratio $(T S R)$ is established as an equivalence between the linear blade-tip velocity and the free-stream velocity:

$$
T S R=\frac{\left|\Omega_{s y s}\right| R_{r t r}}{U_{\infty}}=\frac{\left|\Omega_{x}\right| R_{r t r}}{U_{\infty}}
$$

where $\Omega_{\text {sys }}$ is the system rotational speed, hence $\Omega_{x}$ being the axial angular velocity, and $R_{r t r}$ is the rotor radius. A distinction must be made between the device radius and the rotor radius when modelling ducted turbines. In the case of a ducted turbine, $R_{d v c} \neq R_{r t r}$, but $R_{d v c}=R_{d c t}$, contrary to a non-ducted turbine, where $R_{d v c}=R_{r t r}$.

The rotor solidity $\left(S_{r t r}\right)$ of a turbine is applied to establish a ratio between the projected area of the static rotor in the axial direction $\left(A_{b l d-z}\right)$ and the total area of the rotor rotation $\left(A_{r t r}\right)$ :

$$
S_{r t r}=\frac{A_{b l d-z}}{A_{r t r}}=\frac{A_{b l d-z}}{\pi R_{r t r}^{2}}
$$

The radius-based Reynolds number $\left(R e_{\infty}\right)$ is utilised to acquire a dimensionless representation of the fluid-structure interaction by considering the fluid inertial and viscous forces as a bluff body:

$$
R e_{\infty}=\frac{\rho U_{\infty} R_{d v c}}{\mu}
$$

where $\rho$ is the fluid density, $U_{\infty}$ is the free-stream velocity, and $\mu$ is the fluid dynamic viscosity.

The length-based Reynolds number $\left(R e_{L}\right)$ is utilised to acquire a dimensionless representation of the fluid-structure interaction by considering the fluid inertial and viscous forces along the length of the structure:

$$
R e_{L}=\frac{\rho U_{\infty} L_{d v c}}{\mu}
$$

where $L_{d v c}$ is the device length, and hence is equal either to the hub length $\left(L_{h u b}\right)$ for the HATT, or the duct length $\left(L_{d c t}\right)$ for the ducted turbine.

In continuation, the chord-based Reynolds number $\left(R e_{c h}\right)$ is utilised to acquire a dimensionless representation of the fluid-structure interaction in terms of the resultant velocity at $75 \%$ of the radius of the rotating device blades:

$$
R e_{c h}=\frac{\rho c_{75 \%} \sqrt{\left(U_{\infty}\right)^{2}+\left(\Omega_{x} R_{75 \%}\right)^{2}}}{\mu}
$$

where $c_{75 \%}$ is the chord length at $75 \%$ of the blade radius $\left(R_{75 \%}\right)$. 
To determine the turbine capacity in converting the fluid free-stream energy into rotational energy, the power coefficient $\left(C_{P}\right)$ is established. This considers the mechanical rotational power attained by the device $\left(P_{d v c}\right)$ as a ratio of the maximum rotational power potentially acquired in the device area $\left(P_{\infty}\right)$ :

$$
C_{P}=\frac{P_{d v c}}{P_{\infty}}=\frac{M_{x} \Omega_{x}}{\frac{1}{2} \rho A_{d v c} U_{\infty}^{3}}=\frac{M_{x} \Omega_{x}}{\frac{1}{2} \rho \pi R_{d v c}^{2} U_{\infty}^{3}}
$$

where $M_{x}$ is the axial moment, also referred to as the rotor torque.

In relation to the power generated, the torque coefficient $\left(C_{Q}\right)$ evaluates the mechanical torque attained by the device $\left(Q_{d v c}\right)$ as a ratio of the maximum torque potentially acquired in the device area $\left(Q_{\infty}\right)$ :

$$
C_{Q}=\frac{Q_{d v c}}{Q_{\infty}}=\frac{M_{x}}{\frac{1}{2} \rho A_{d v c} R_{r t r} U_{\infty}^{2}}=\frac{M_{x}}{\frac{1}{2} \rho \pi R_{d v c}^{2} R_{r t r} U_{\infty}^{2}}
$$

In relation to the power generated, the mass flow-rate coefficient $\left(C_{\dot{m}}\right)$ evaluates the mass flow-rate through the rotor $\left(\dot{m}_{r t r}\right)$ as a ratio of the maximum mass flow-rate which may be potentially attained within the duct:

$$
C_{\dot{m}}=\frac{\dot{m}_{r t r}}{\rho A_{r t r} U_{\infty}}=\frac{\dot{m}_{r t r}}{\rho \pi R_{r t r}^{2} U_{\infty}}
$$

In continuation, the resultant thrust on the device induced in a direction parallel to the turbine axis contributes to the fluid-structure phenomenon. The thrust coefficient $\left(C_{T}\right)$ quantifies this as a function of the device thrust $\left(T_{d v c}\right)$ and the maximum thrust potentially induced upon the device area $\left(T_{\infty}\right)$ :

$$
C_{T}=\frac{T_{d v c}}{T_{\infty}}=\frac{F_{x}}{\frac{1}{2} \rho A_{d v c} U_{\infty}^{2}}=\frac{F_{x}}{\frac{1}{2} \rho \pi R_{d v c}^{2} U_{\infty}^{2}}
$$

where $F_{x}$ is the stream-wise axial force on the device.

In analysing the transient sinusoidal fluctuations in the performance outcomes, Strouhal's law was utilised in evaluating its relationship with the Reynolds number of the domain:

$$
S t=\frac{f_{v} L_{S t}}{U_{\infty}}
$$

where $S t$ is the Strouhal number, $f_{v}$ is the vortex-shedding frequency, and $L_{S t}$ is the Strouhal characteristic length.

\subsection{CFD Modelling}

Various degrees of flow complexities are induced as a result of the high Reynolds number ranges that large-scale tidal turbines physically approach. These ranges of turbulence are wholly elaborated by random fluctuations of the flow properties. Mean derivatives of the continuity and Navier-Stokes equations are utilised to model these flow properties, expressed as a vectorial summation of their mean and fluctuating terms, which, in turn, are equated by turbulence models to achieve mathematical closure. 


\subsubsection{Conservation Modelling}

Within three-dimensional Cartesian space, the conservation of mass is represented, in indicial notation, as:

$$
\frac{\partial \bar{\rho}}{\partial t}+\frac{\partial}{\partial x_{i}}\left(\bar{\rho} \tilde{u}_{i}\right)+\frac{\partial}{\partial x_{j}}\left(\bar{\rho} \tilde{u}_{j}\right)+\frac{\partial}{\partial x_{k}}\left(\bar{\rho} \tilde{u}_{k}\right)=0
$$

where $\bar{\rho}$ is the Reynolds-averaged density, $x_{i}$ is the Cartesian coordinate, $t$ is the elapsed time, and $\tilde{u}_{i}$ is the mass-averaged velocity $[35,36]$.

Assuming the fluid is incompressible, single-phase, and isotropic, due to the physicality of the hydrodynamic domain, within a Reynolds-averaged, tensor notation, the mass continuity equation takes the form of:

$$
\frac{\partial U_{i}}{\partial x_{i}}=0
$$

where $U_{i}$ is the Reynolds-averaged velocity.

In continuation, the conservation of momentum within three-dimensional, tensor notation is represented as:

$$
\frac{\partial\left(\bar{\rho} \tilde{u}_{i}\right)}{\partial t}+\frac{\partial}{\partial x_{j}}\left(\bar{\rho} \tilde{u}_{i} \tilde{u}_{j}-\bar{\sigma}_{i j}\right)=0
$$

where $\bar{\sigma}_{i j}$ is the total stress tensor. The above equation, in Reynolds-averaged format, then becomes:

$$
\rho \frac{\partial U_{i}}{\partial t}+\rho \frac{\partial}{\partial x_{j}}\left(U_{i} U_{j}+\overline{u_{j}^{\prime} u_{i}^{\prime}}\right)-\frac{\partial}{\partial x_{j}}\left(\sigma_{i j}\right)=0
$$

where $U_{i}$ is the Reynolds-averaged velocity, $u_{i}^{\prime}$ is the fluctuating velocity component, and $\sigma_{i j}$ is the Reynolds-averaged total stress tensor, which, for incompressible flows, under the absence of volume dilation (or elastic stress), is specified as the tensor summation of the static pressure and viscous stress tensor:

$$
\sigma_{i j}=-\delta_{i j} P_{s}+\mu\left(\frac{\partial U_{i}}{\partial x_{j}}+\frac{\partial U_{j}}{\partial x_{i}}\right)
$$

where $\delta_{i j}$ is the Kronecker delta (or unit tensor), $P_{s}$ is the static pressure, and $\mu$ is the dynamic viscosity. The momentum equation, therefore, may be presented as:

$$
\rho \frac{\partial U_{i}}{\partial t}+\rho U_{j} \frac{\partial U_{i}}{\partial x_{j}}=-\frac{\partial P_{s}}{\partial x_{i}}+\frac{\partial}{\partial x_{j}}\left(\mu\left(\frac{\partial U_{i}}{\partial x_{j}}+\frac{\partial U_{j}}{\partial x_{i}}\right)-\rho \overline{u_{j}^{\prime} u_{i}^{\prime}}\right)
$$

where $-\rho \overline{u_{j}^{\prime} u_{i}^{\prime}}=\rho \tau_{i j}$, and is known as the Reynolds-stress tensor, with $\tau_{i j}=-\overline{u_{j}^{\prime} u_{i}^{\prime}}$ being the specific Reynolds-stress tensor. 


\subsubsection{Turbulence Modelling}

In introducing mathematical capacities to model the specific Reynolds-stress tensor, and hence, model turbulence within the computational domain, measures are implemented to regard parametric effects of flow history transience, together with the development of convection, production, and body-force terms, in response to physical disparities in strain rate, and the resultant diffusion, redistribution, and dissipation [31].

In an effort to introduce the aspects of turbulence, the Wilcox Stress- $\omega$ turbulence model was utilised in this CFD model, in which the specific Reynolds-stress tensor $\left(\tau_{i j}\right)$, in tensor notation, is established by:

$$
\begin{aligned}
\rho \frac{\partial \tau_{i j}}{\partial t}+\rho U_{k} \frac{\partial \tau_{i j}}{\partial x_{k}}= & \frac{\partial}{\partial x_{k}}\left[\left(\mu+\frac{\mu_{T}}{\sigma_{k}}\right) \frac{\partial \tau_{i j}}{\partial x_{k}}\right]-\rho P_{i j}-\rho \Pi_{i j}+ \\
& \frac{2}{3} \beta^{*} \rho \omega k \delta_{i j}-2 \rho \omega_{k}\left(\tau_{j m} \epsilon_{i k m}+\tau_{i m} \epsilon_{j k m}\right)
\end{aligned}
$$

where $\rho$ is the Reynolds-averaged density, $P_{i j}$ is the Reynolds stress production tensor, $t$ is the elapsed time, $x_{i}$ is the Cartesian coordinate, $k$ is the kinetic energy of turbulent fluctuations per unit mass (or turbulence kinetic energy), $\omega$ is the specific dissipation rate, $\Pi_{i j}$ is the pressure-strain correlation tensor, $\mu$ is the dynamic viscosity, and $\mu_{T}$ is the eddy viscosity, $\omega_{k}$ is the rotation vector, and $\epsilon_{i j k}$ is the Levi-Civita pseudotensor; $\beta^{*}$ and $\sigma_{k}$ are closure coefficients.

The specific dissipation rate $(\omega)$ is defined by:

$$
\rho \frac{\partial \omega}{\partial t}+\rho U_{j} \frac{\partial \omega}{\partial x_{j}}=\frac{\partial}{\partial x_{j}}\left[\left(\mu+\frac{\mu_{T}}{\sigma_{k}}\right) \frac{\partial \omega}{\partial x_{j}}\right]+\alpha \frac{\rho \omega}{k} \tau_{i j} \frac{\partial U_{i}}{\partial x_{j}}-\beta_{o} f_{\beta} \rho \omega^{2}
$$

where $f_{\beta}$ is utilised to implement the mean rotation tensor $\left(\Omega_{i j}\right) ; \alpha$ and $\beta_{o}$ are closure coefficients.

The pressure-strain correlation tensor $\left(\Pi_{i j}\right)$ is defined by:

$$
\Pi_{i j}=\beta^{*} C_{1} \omega\left(\tau_{i j}+\frac{2}{3} k \delta_{i j}\right)-\hat{\alpha}\left(P_{i j}-\frac{1}{3} P_{k k} \delta_{i j}\right)-\hat{\beta}\left(D_{i j}-\frac{1}{3} P_{k k} \delta_{i j}\right)-\hat{\gamma} k\left(S_{i j}-\frac{1}{3} S_{k k} \delta_{i j}\right)
$$

where $S_{i j}$ is the mean strain-rate tensor and $D_{i j}$ is the rotation production tensor; $C_{1}, \hat{\alpha}, \hat{\beta}$, and $\hat{\gamma}$ are closure coefficients.

Specificities with regards to tensor definitions, and closure coefficients and derivatives may be further established in the referenced literature [31, 35].

\section{Performance Validation of a Horizontal-Axis Tidal Turbine}

In validation of the CFD model, the numerical outcomes of a three-bladed HATT within a TSR range of $1.00-8.26$ in open-water conditions, in relevance to the power coefficient, torque coefficient, thrust coefficient, and wake velocity profiles from near- to far-wake, were ascertained in comparison to Mycek et al. [32] utilising the coefficient of determination $\left(R^{2}\right)$ statistical methodology. 


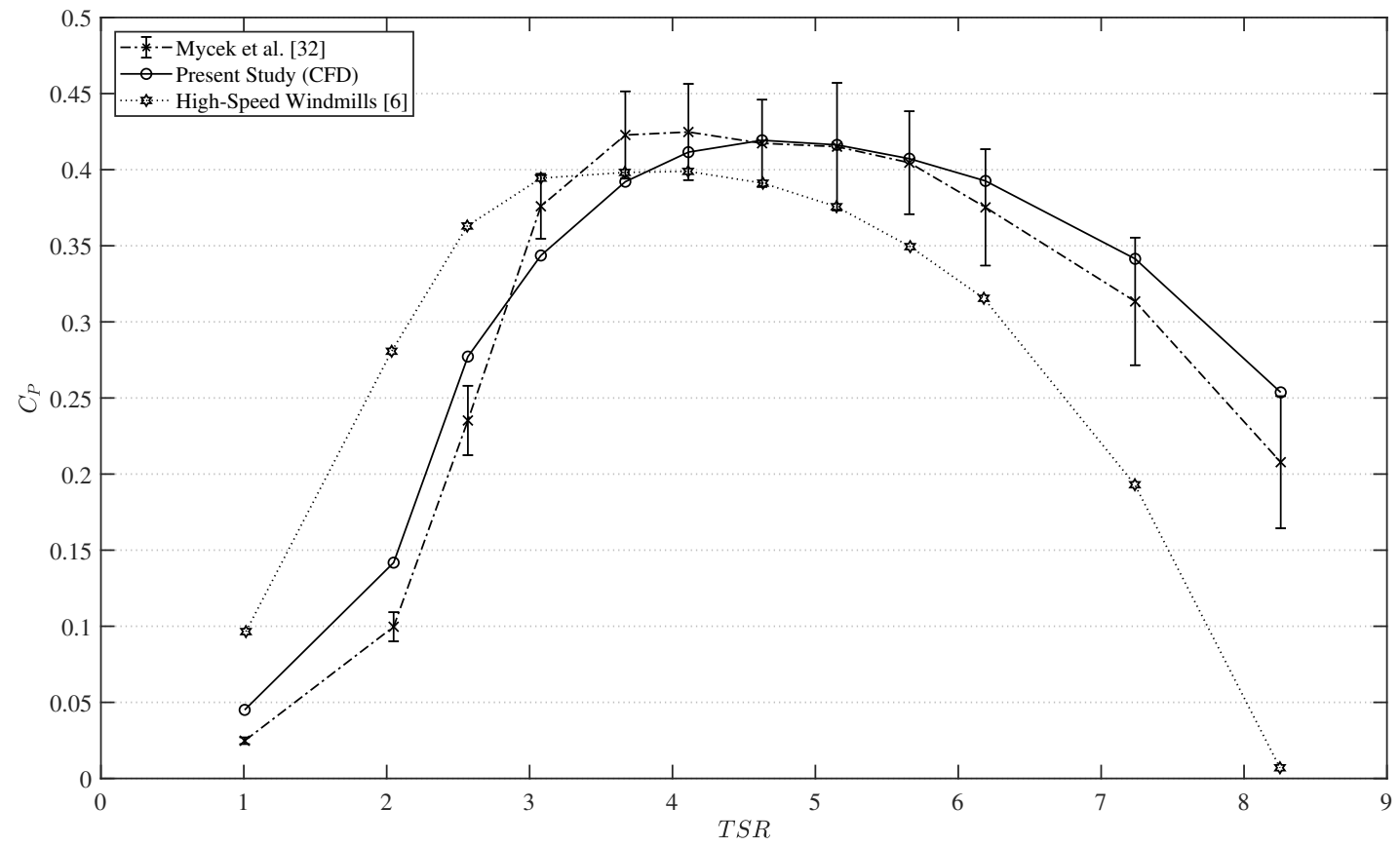

(a) Power coefficient $\left(C_{P}\right)$ in relation to TSR

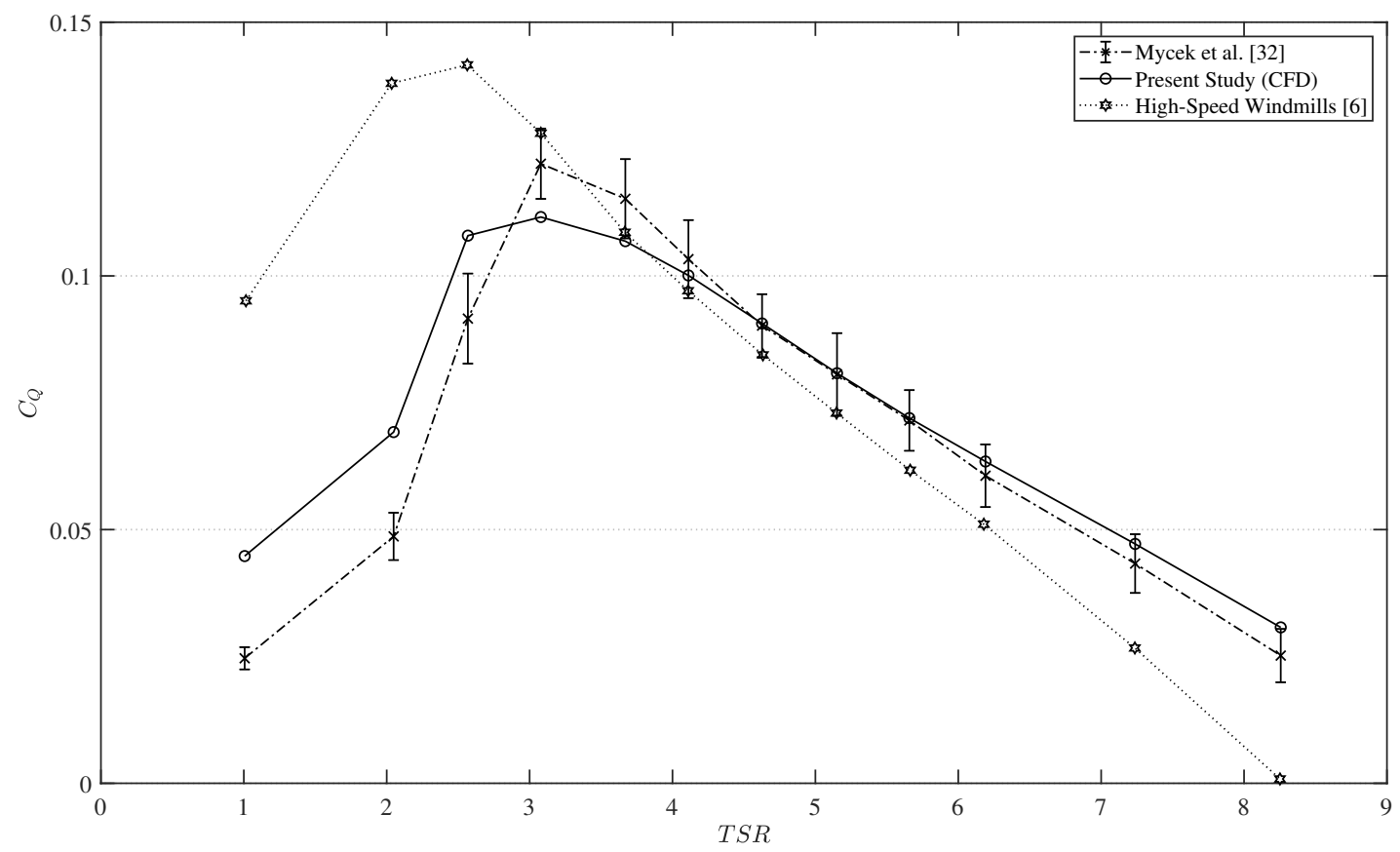

(b) Torque coefficient $\left(C_{Q}\right)$ in relation to TSR

Figure 5: Comparative evaluation of the current horizontal-axis tidal turbine CFD model with experimentation [32], and high-speed windmills [6] 


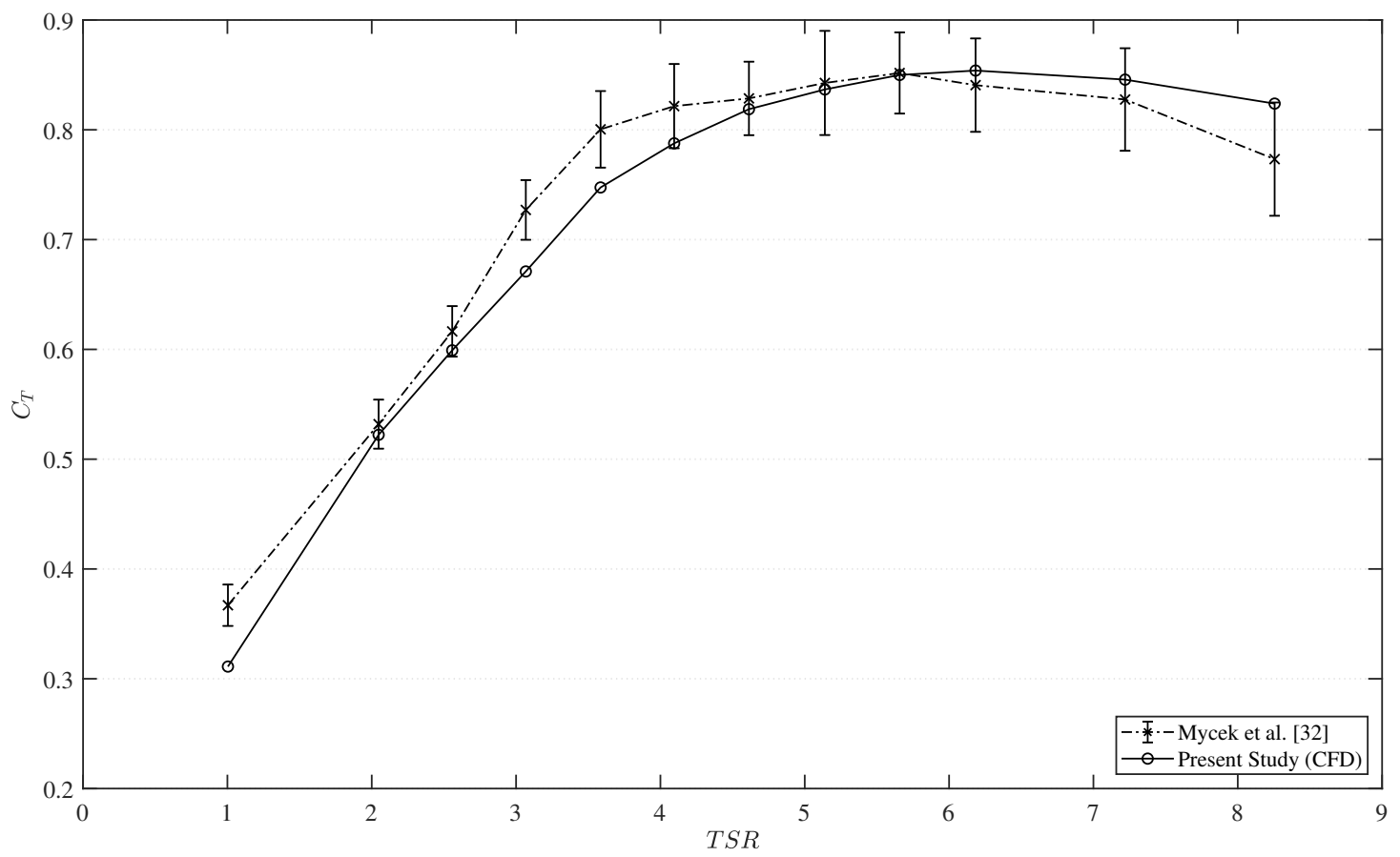

Figure 6: Comparative evaluation of the current horizontal-axis tidal turbine CFD model with experimentation [32] for thrust coefficient $\left(C_{T}\right)$ in relation to TSR

\subsection{Power Coefficient}

Primarily, the power coefficient $\left(C_{P}\right)$ curve established by numerical means, illustrated in Figure 5a, attained an $R^{2}$ of 0.955 as a degree of similarity with experimentation pertaining to the data points within the entire curve, and a 0.965 degree of similarity within the nominal TSR range (TSR 3.08 - 5.66). As the limits of the error bars in the graphs represent one standard deviation $(\sigma)$, all CFD data points analysed fell within $2 \sigma_{C_{P}}$ of the experimentation points, hence within the $95^{t h}$-percentile confidence range, except for low TSR values of 1.00 and 2.05. Furthermore, the torque coefficient $\left(C_{Q}\right)$ curve established by numerical means, illustrated in Figure 5b, attained an $R^{2}$ of 0.889 as a degree of similarity with experimentation pertaining to the data points within the entire curve, and a 0.965 degree of similarity within the nominal TSR range (TSR 3.08 - 5.66). All CFD data points analysed fell within $2 \sigma_{C_{Q}}$ of the experimentation points, hence within the $95^{t h}$-percentile confidence range, except for low TSR values of 1.00 and 2.05. Specifying the affiliated physics between the validation systems and large-scale systems, the numerical and experimentation data sets are compared to high-speed windmills presented in the Betz [6] technical memorandum, indicating good comparison specifically within the trends of the performance curves.

\subsection{Thrust Coefficient}

In continuation, the thrust coefficient $\left(C_{T}\right)$ curve established by numerical means, in consideration of the rotor, nacelle, and mast, illustrated in Figure 6 , attained an $R^{2}$ of 


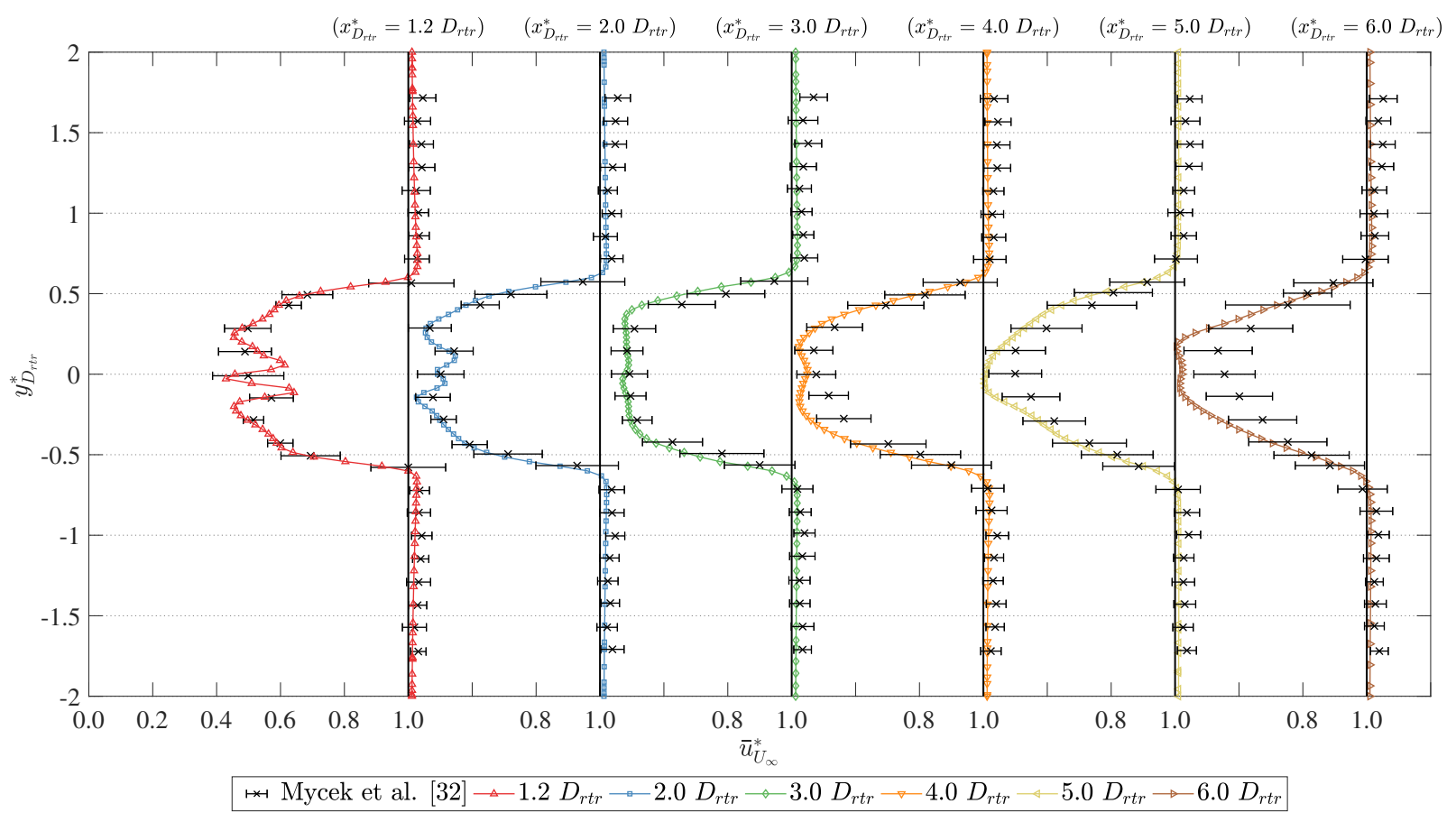

Figure 7: Comparative evaluation of the current horizontal-axis tidal turbine CFD model with experimentation [32] for the wake velocity profiles at distinct downstream displacements $\left(x_{D_{r t r}}^{*}\right)$ at TSR 3.67

0.946 as a degree of similarity with experimentation pertaining to the data points within the entire curve, and a 0.841 degree of similarity within the nominal TSR range (TSR 3.08 - 5.66). All CFD data points analysed fell within $2 \sigma_{C_{T}}$ of the experimentation points, hence within the $95^{\text {th }}$-percentile confidence range, except for the low TSR value of 1.00. The comparatively substantial discrepancy between the numerical and experimentation thrust coefficient may have come about due to the inaccuracy of the mast geometry implemented within the model. No data regarding the position and dimensions of the mast were noted in the literature, and hence, creating the complete geometry was executed with calculated estimations; in addition, fairings were utilised during the experimentation, whereas a bare mast was created in the numerical model, hence skewing the resultant data comparison.

\subsection{Wake Velocity Profiles}

Further to the performance evaluation, the wake velocity profiles established by numerical means, illustrated in Figure 7 , attained an $R^{2}$ of 0.982 and 0.863 as a degree of similarity with experimentation pertaining to the data points within the entire curve, at near-wake and farwake respectively. All the CFD data points analysed fell within $2 \sigma_{C_{T}}$ of the experimentation points, hence within the $95^{\text {th }}$-percentile confidence range. The comparatively substantial discrepancy between the numerical and experimentation at the far-wake may be a result of the underestimation of the dissipation rate within the fluid, due to the implementation of the turbulence model, hence overestimating the resultant kinetic energy within the wake. 


\section{Performance of the Ducted High-Solidity Tidal Turbine}

As a high degree of similarity between the dynamic rotor properties was attained through the validation process, it was ascertained that the modelling approach and turbulence model implemented were of appropriate physical representation for a rotating bladed system; in recognition, the numerical schemes were adopted for the analysis of the ducted tidal turbine. By this means, in relation to studies that have endeavoured in modelling ducted tidal turbines $[4,5,28]$, this present study produces a more coherent representation of the configuration, both due to the accurate geometrical representation, and in the numerical modelling of general conservation and turbulence, hence better analysing the hydrodynamic occurrence of the fluid-structure interaction.

\subsection{Power Coefficient}

Upon implementation of the validated modelling techniques for the ducted turbine model, unique physical performance outcomes were displayed in representation of its high-solidity characteristics. Notably, the power coefficient TSR curve is relatively short spanning, with a TSR range of $1.00-2.50$ to achieve a $C_{P}$ decrease of 0.05 from the nominal TSR. Within this region, a maximum power coefficient range between 0.335 and 0.346 , at free-stream velocities of $1 \mathrm{~m} / \mathrm{s}$ and $7 \mathrm{~m} / \mathrm{s}$ respectively, was achieved, as illustrated in Figure 8 . Throughout the free-stream velocity variation, the power coefficient decrease is largely consistent postnominal TSR. The nominal TSR point, however, differs at distinctive velocities. At low free-stream velocities $\left(U_{\infty}=1 \mathrm{~m} / \mathrm{s}\right)$, the nominal TSR lies at 1.50 , whereas at higher velocities $\left(U_{\infty}=2.5-7 \mathrm{~m} / \mathrm{s}\right)$, the nominal TSR was found to shift to 1.75 . This variation in nominal TSR was found to be in consequence of the angle-of-attack $\left(\alpha_{A o A}\right)$ attained by the differing hydrofoils composing the blade.

As part of the work detailed in the thesis, Allsop [3] investigated the effect of the variation of chord-based Reynolds number on the hydrodynamic performance coefficients of the flatplate hydrofoil sections comprising the high-solidity blade utilising CFD. By considering a Reynolds number range of $1 \cdot 10^{6}$ to $1 \cdot 10^{7}$, a transition in the hydrofoil stall angle was depicted, increasing from an angle of $11^{\circ}$ to that of $14^{\circ}$, respectively. As the distinct flat-plate hydrofoil sections constitute the full blade, the effect of this stall angle transition is depicted in the power coefficient curve. At low TSR, the angle of attack at distinct elements within the blade is considerably high, inducing flow separation due to boundary layer detachment along the downstream surface, resulting in a region of the blade to experience stall. With an increase in TSR, the angle of attack decreases to the stall threshold, where the highest lift coefficient value is attained. Within this flow range, the boundary layer sticks to, and flows along, the section surface, resulting in the region to come out of stall. At this flow condition, optimal hydrodynamic parameters are induced, which characterises the criteria for peak power, occurring at optimal TSR. When this threshold is surpassed, a reduction in power is instigated in correlation to a steep linear decline in the lift parameter response of the flat-plate hydrofoil. The stall threshold is proportional to the chord-based Reynolds number, and hence, due to the shift in angle-of-attack, the optimal TSR correspondingly shifts to a higher TSR value at higher Reynolds numbers. Simulating more domain flow 


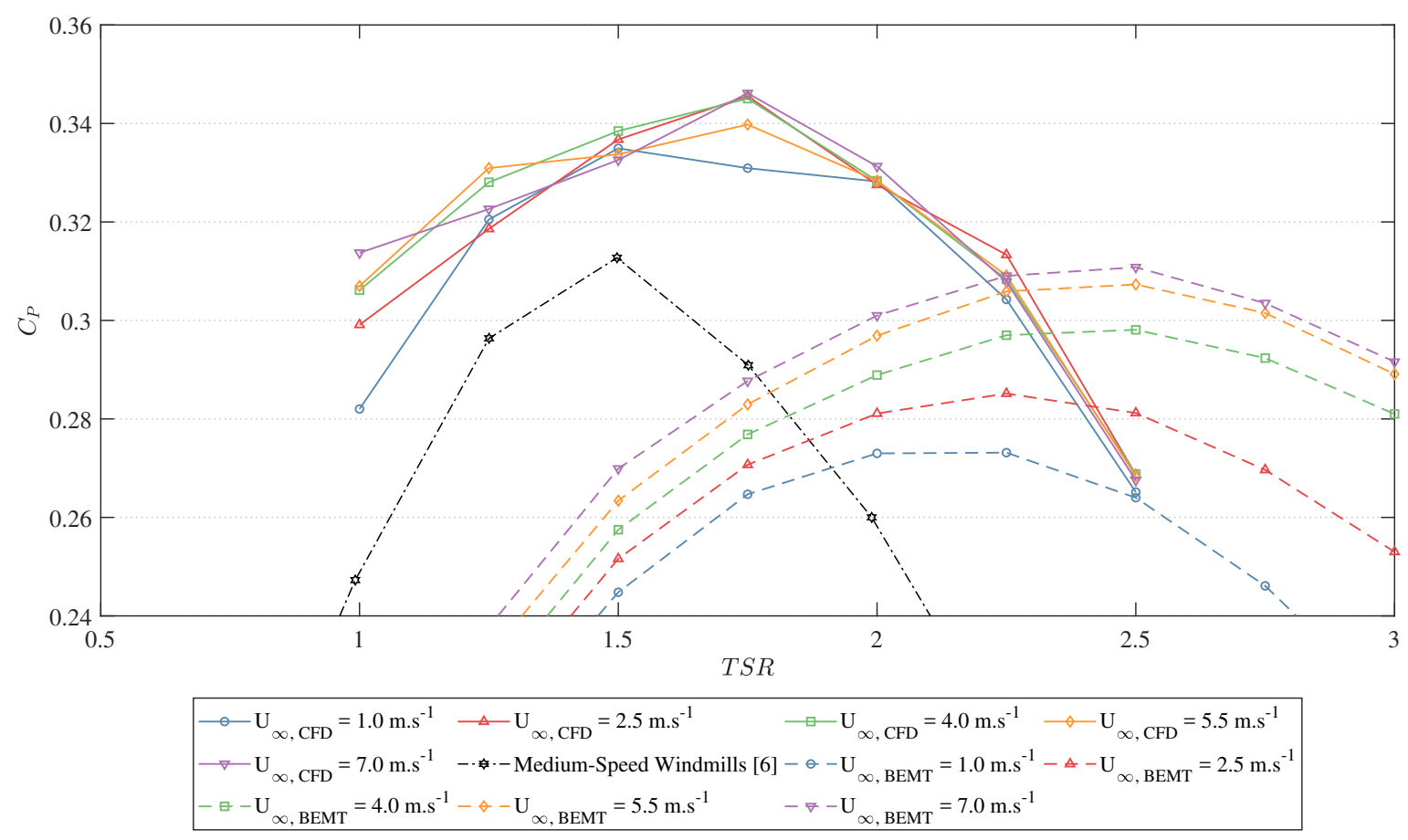

Figure 8: Evaluation of the mean ducted turbine power coefficient $\left(C_{P}\right)$ in relation to TSR

parameters would enable the identification of the definite TSR at which the blade is within the peak lift region, hence the point at which the degree of blade area within the stall region reaches tipping point. The physical value of this output, however, may be limited due to the sensitive nature of the rotating blade boundary layer, together with external influences from the turbulent and multi-directional nature of the tidal environment.

In accordance to the hydrodynamic performance characteristics, albeit a maximum power coefficient of 0.346 was attained, it may also be acknowledged that, due to the device area $\left(A_{d v c}\right)$ being considered, hence rotor \& duct, the power coefficient will increase once the power per rotor area $\left(A_{r t r}\right)$, rather than system area, is acknowledged. In this respect, the rotor radius is $80 \%$ that of the system, hence increasing the maximum power coefficient by a magnitude of 1.5625 to a nominal peak value of 0.541 . Due to the fact, however, that the power attained by the rotor is also disseminated from the fluid in contact with the duct, the entire system area is a more conservative approach to represent.

In comparison to BEMT modelling elaborated in [3], an areal discrepancy was acknowledged over the TSR range, and achieved moderately comparable figures at nominal TSR, with a discrepancy in evaluation of the maximum $C_{P}$, which ranges from 0.273 to 0.311 , at the free-stream velocities considered. This discrepancy may be elucidated by the dissimilarities in the hydrodynamic modelling of the two numerical techniques. Notably, the BEMT method considers the turbine blade as an array of discrete hydrofoil sections, considerably reducing the computational expense, where the hydrodynamic parameters of the blades are attained by means of two-dimensional CFD analyses. This approach, however, neglects any span-wise flow interactions along the blade length, together with omitting complex flow sep- 


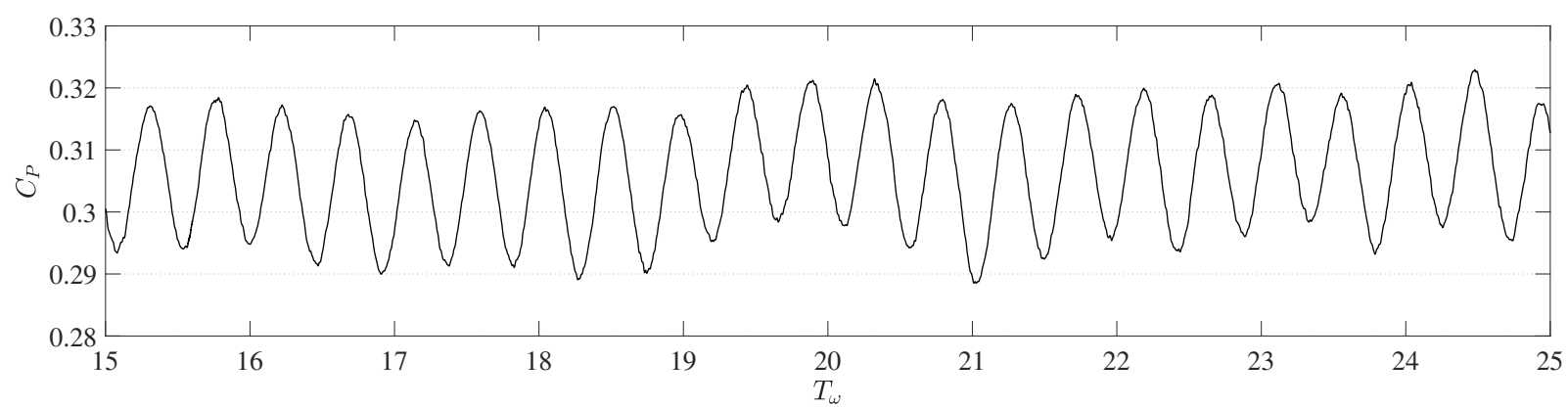

(a) $T S R 1.00$

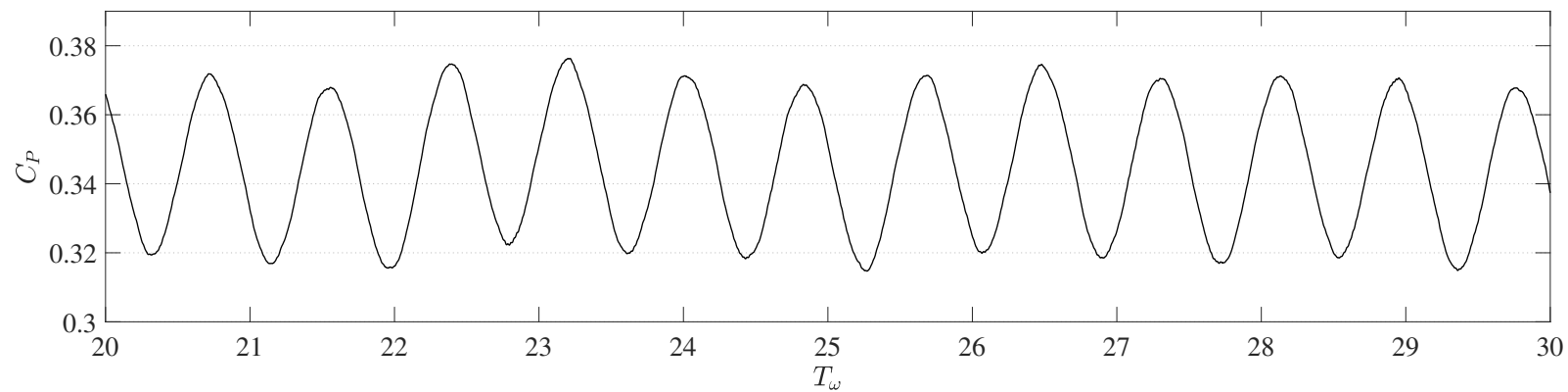

(b) TSR 1.75

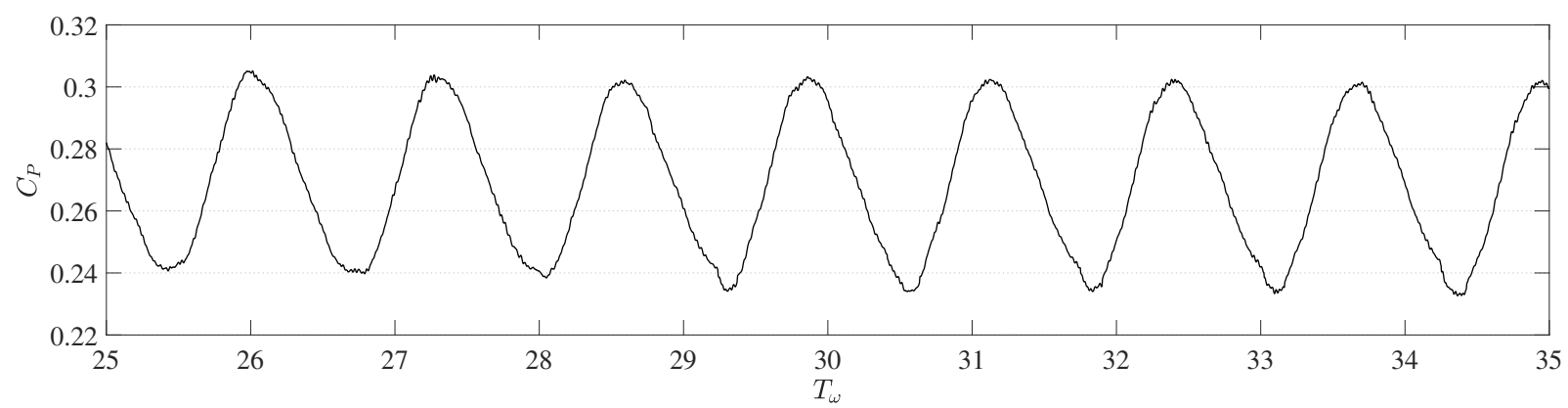

(c) TSR 2.50

Figure 9: Representation of the transient power coefficient output at $U_{\infty}=4.0 \mathrm{~m} / \mathrm{s}$ through turbine rotary periods $\left(T_{\omega}\right)$

aration at the open-centre blade tips, which are considered to be significant fluid dynamic features. The latter phenomenon is accounted for in BEMT by implementing the Prandtl tip loss factor, yet is better captured when utilising blade-resolved CFD. Albeit the drawbacks of the BEMT methodology, significantly accurate outputs are attained in consideration of the inputs provided within the numerical model contrived and the computational duration required.

Whilst presenting windmill findings in the technical memorandum, Betz [6] described a "medium-speed windmill" that attains maximum efficiency at a low TSR value within a short-spanning TSR curve. Potentially resembling the OpenHydro PS2 tidal turbine, a comparison was made between the reported findings and the CFD results, also illustrated 


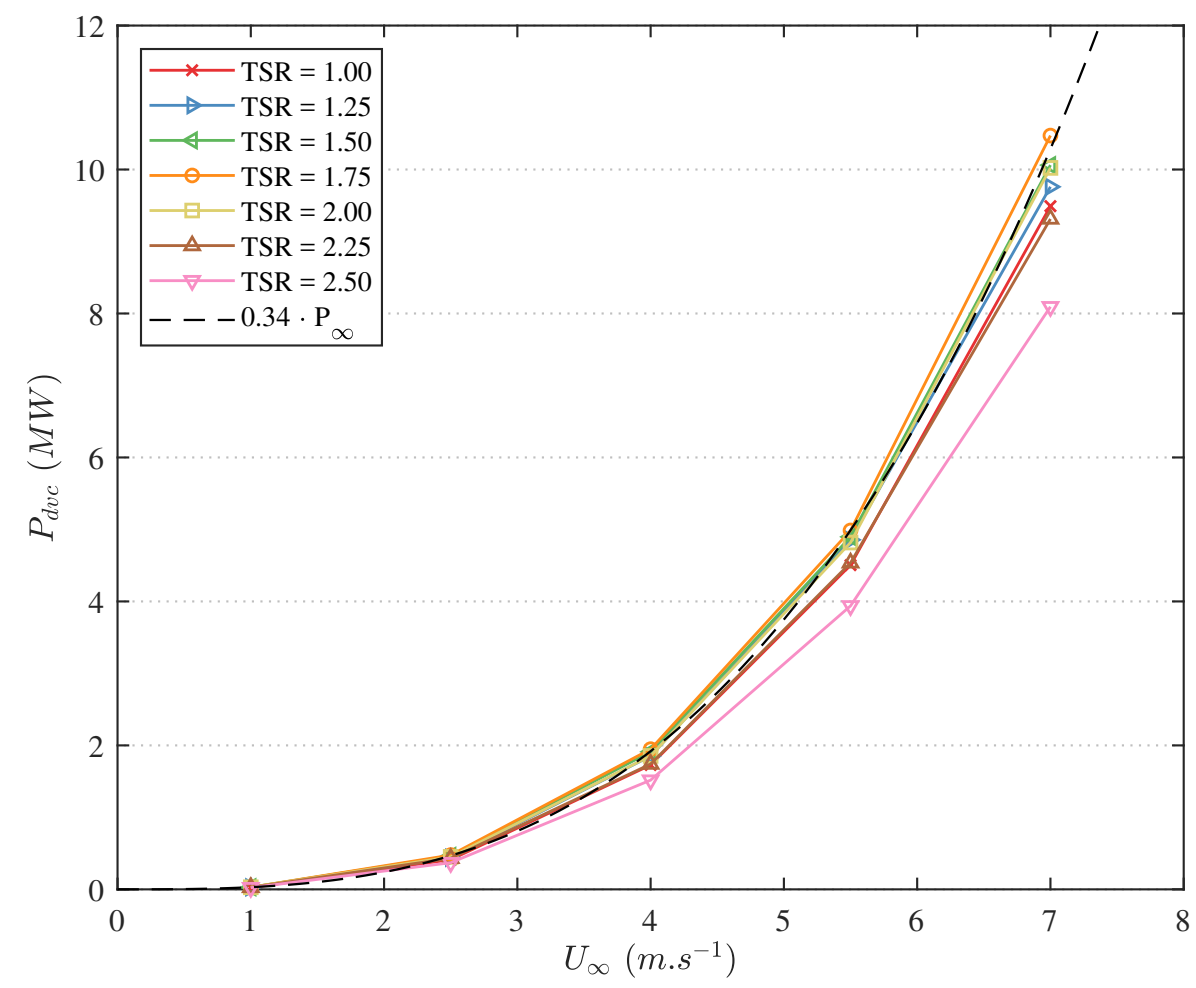

Figure 10: Evaluation of the ducted turbine mechanical power $\left(P_{d v c}\right)$ in relation to free-stream velocity $\left(U_{\infty}\right)$ at distinct TSRs

in Figure 8. With a peak windmill power coefficient of 0.313 at a nominal TSR of 1.50, good agreement was acknowledged in the parameter trend when compared to the ducted high-solidity turbine system.

In continuation, to comprehend the physical representation of the power generated by the turbine, Figure 10 illustrates the variation in the power output with the velocity of the free-stream. In comparison to the theoretical representation of a power curve, the peak power coefficients at nominal TSR $(T S R=1.75)$ at varying velocities match well with a mean power coefficient of 0.34 . Further to the power curve, the variation in the torque coefficient $\left(C_{Q}\right)$ induced on the turbine is illustrated in Figure 11. Similar areal discrepancies are attained in comparison to BEMT and the Betz curve, yet, albeit the differences, the key similarity between the comparisons is the trend at which the torque varies.

In consideration of the substantial correspondence between the power \& torque coefficient performance curves displayed within the CFD results, BEMT outputs, and windmill (experimentation) findings, the applicability of full-scale analysis by means of the methodology implemented was acknowledged. The total discrepancy, however, both in comparison to windmill findings and BEMT outputs, may be a result of the over-sensitivity of the implemented turbulence model physics. This, ultimately, may overestimate the pressure distribution on the wall-bounded conditions, and hence, mechanical power transition due to 


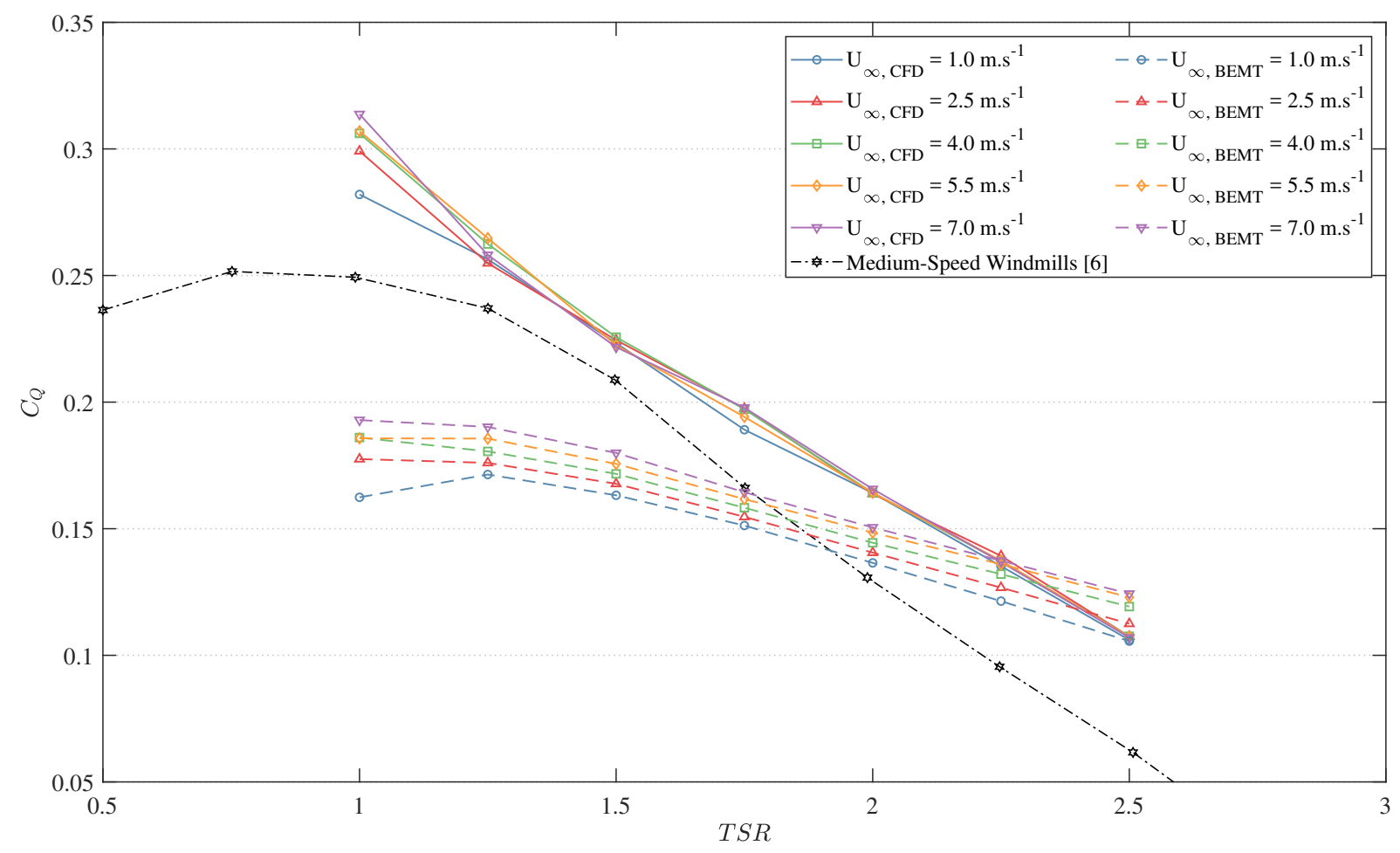

Figure 11: Evaluation of the ducted turbine torque coefficient $\left(C_{Q}\right)$ in relation to TSR

the shear flow induced at the surfaces of the blades; further analyses utilising differing turbulence models may be required to ascertain this outcome. In definition of the parametric outcomes, the imperative of executing both CFD and BEMT methodologies when analysing turbine performance is exemplified. A denomination of the induced physical effects are more appropriately identified by means of a simplified model, and may be of assistance in understanding the fluid dynamic outcomes of higher complexity analyses, such as that described within this work, due to the model capacity in capturing multiple coupled effects, such as lateral flow interactions down the blade length and temporal fluctuations in the rotor performance, as depicted in Figure 9, where the individual physical phenomena inducing the hydrodynamic behaviour are less obvious.

\subsection{Thrust Coefficient}

The implementation of a duct along the perimeter of a turbine subsequently increases the area onto which an external force may be induced, potentially bringing about an intensification in physical bluff-body conditions. In effect, the combined drag induced upon the system becomes of particular interest.

Illustrated in Figure 12, the variation in thrust coefficient $\left(C_{T}\right)$ with TSR and freestream velocity is presented both in terms of the entire system, and in terms of the physical components: rotor and duct. As an entire system, a substantially high thrust coefficient is attained at low TSR, a mean value of 1.17, which decreases to a mean of 0.70 at high TSR. The gradient acts in a polynomial manner to nominal TSR, where the mean thrust coefficient 
is precisely 1.00, after which the gradient acts linearly. Observed from the component curves, this considerable discrepancy in drag is largely consistent upon both the duct and the rotor, with a mean allocation of the global drag of approximately $32 \%$ and $68 \%$, respectively. The rotor hence succumbs to roughly two-thirds of the global drag, whilst the duct succumbs to one-third; this allocation is somewhat relative to the areas of the duct and rotor, being $36 \%$ and $60 \%$ of the system area, respectively. In comparison to BEMT, which solely considers the drag on the rotor, a significant discrepancy is attained between the two methodologies, albeit exhibiting a moderately similar diminishing $C_{T}$ trend.

In effort of establishing the source of the substantial drag on the rotor, the static pressure coefficient $\left(C_{P r}=\frac{P_{s}}{\frac{1}{2} \rho U_{\infty}^{2}}\right)$ distributions within the domain at the vicinity of the rotor were qualitatively analysed. Principally, the induction of axial drag upon a structure is a straightforward concept; pressure upstream and suction downstream of the structure induces this outcome. Illustrated in Figure 13, at low TSR, the static pressure upstream and the suction downstream of the rotor are substantial. Acknowledging the flow-rate through the duct, at low TSR, the flux is comparatively low, as illustrated in Figure 14. As a result, as the freestream comes into constant contact with the structure, fluid accumulates at the inlet of the duct, upstream of the rotor, hence increasing the static pressure in this region. Furthermore, due to the high-solidity of the rotor, insufficient fluid is permitted to flow through the duct, hence creating a region of low pressure. The duct sustains this variation in pressure as the upstream duct section confines the flow in the vicinity of the rotor, disallowing its leakage, hence inducing a pressure build-up, whilst, directly downstream of the rotor, the duct disallows the free-stream from re-energising the wake, hence sustaining the low pressure zone. As TSR increases, the flow rate through the duct proportionally increases, hence decreasing the static pressure difference fore and aft of the rotor, subsequently reducing drag on both the rotor and the duct; BEMT does not incorporate this fluid dynamic induction within its methodology, hence expounding the discrepancy observed. The variation in mass flow-rate through the turbine, which attains a mean coefficient of 0.680 at low TSR, and increases to a mean of 0.715 and 0.805 at nominal and high TSR, respectively, inversely proportional to the resultant thrust, indicates the substantial influence in the total drag induced upon the entire structure. In consequence to the extensive capacity in mass-flow rate development, due to its high-solidity properties, as TSR is further increased, a negative pressure difference is eventually induced, resulting in the turbine to act as a propeller.

In addition to the system drag principally comprising of the rotor drag, the force on the duct, apart from also being effected by the sustained pressure at the rotor, is dependent on the fluid-structure interaction at the profile of the duct structure. As the external fluid flow is in contact with the entire structure, a significant degree of static pressure builds up at the walls of the duct inlet. This consequently affects the incoming flow, as the far-flow in the direction of the system takes the path of least resistance, and hence bypasses the duct inlet, and flows along the duct outer surface without any interaction with the internal structure. Fluid in the vicinity of the inner duct ridges, however, impacts the internal structure, and interacts with the duct by flowing along the inner duct curvature, either against the freestream, in an upstream direction away from the rotor, or with the free-stream, towards the 

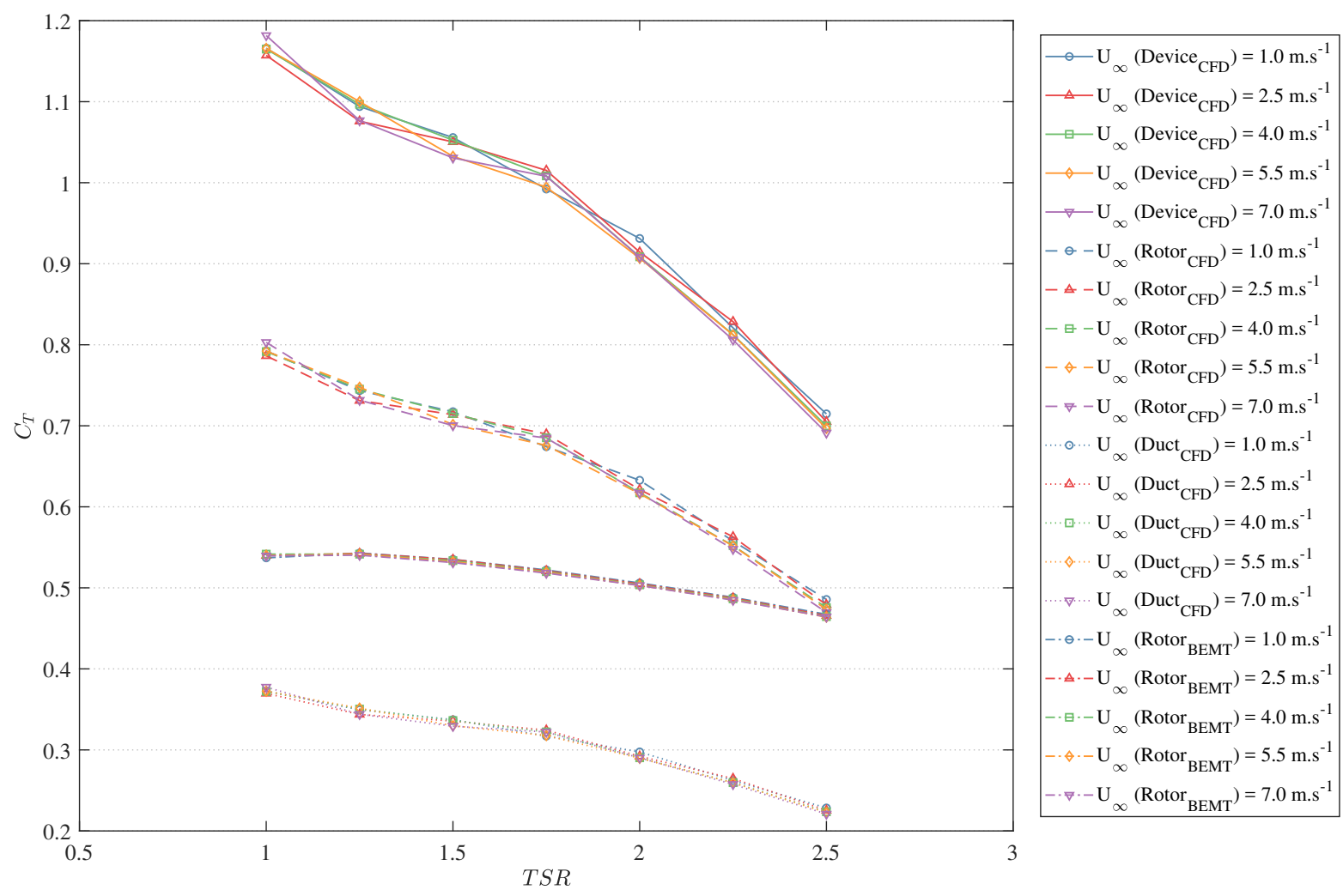

Figure 12: Evaluation of the ducted turbine thrust coefficient $\left(C_{T}\right)$ in relation to TSR

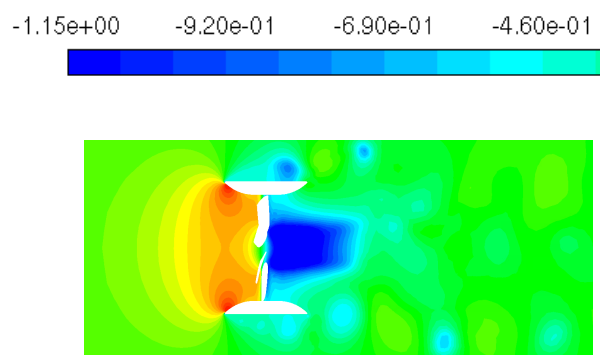

$0.5 T_{\omega}$

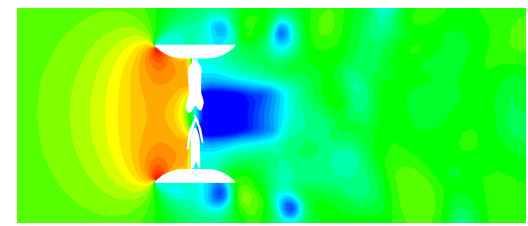

$1.0 T_{\omega}$

(a) $T S R=1.00$

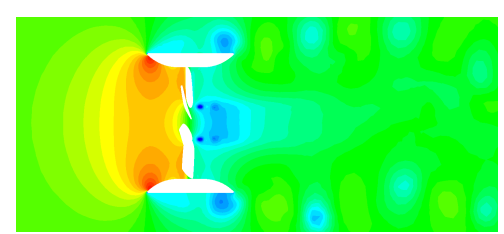

$0.5 T_{\omega}$

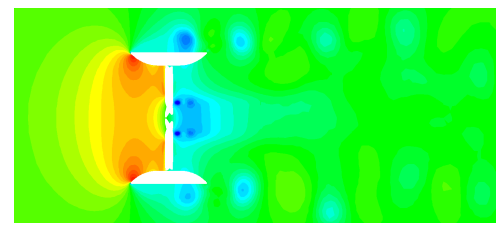

$1.0 T_{\omega}$

(b) $T S R=1.75$

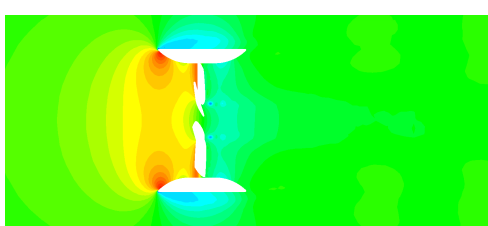

$0.5 T_{\omega}$

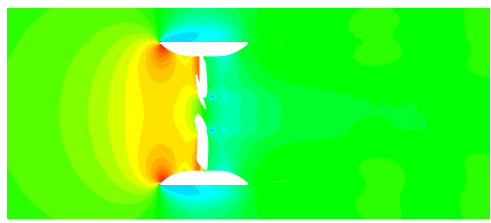

$1.0 T_{\omega}$

(c) $T S R=2.50$

Figure 13: Illustrative top-view representation of the pressure coefficient $\left(C_{P r}\right)$ within the turbine domain at low, nominal, and high $T S R$ at mean-average output; $U_{\infty}=4 \mathrm{~m} / \mathrm{s}$ 


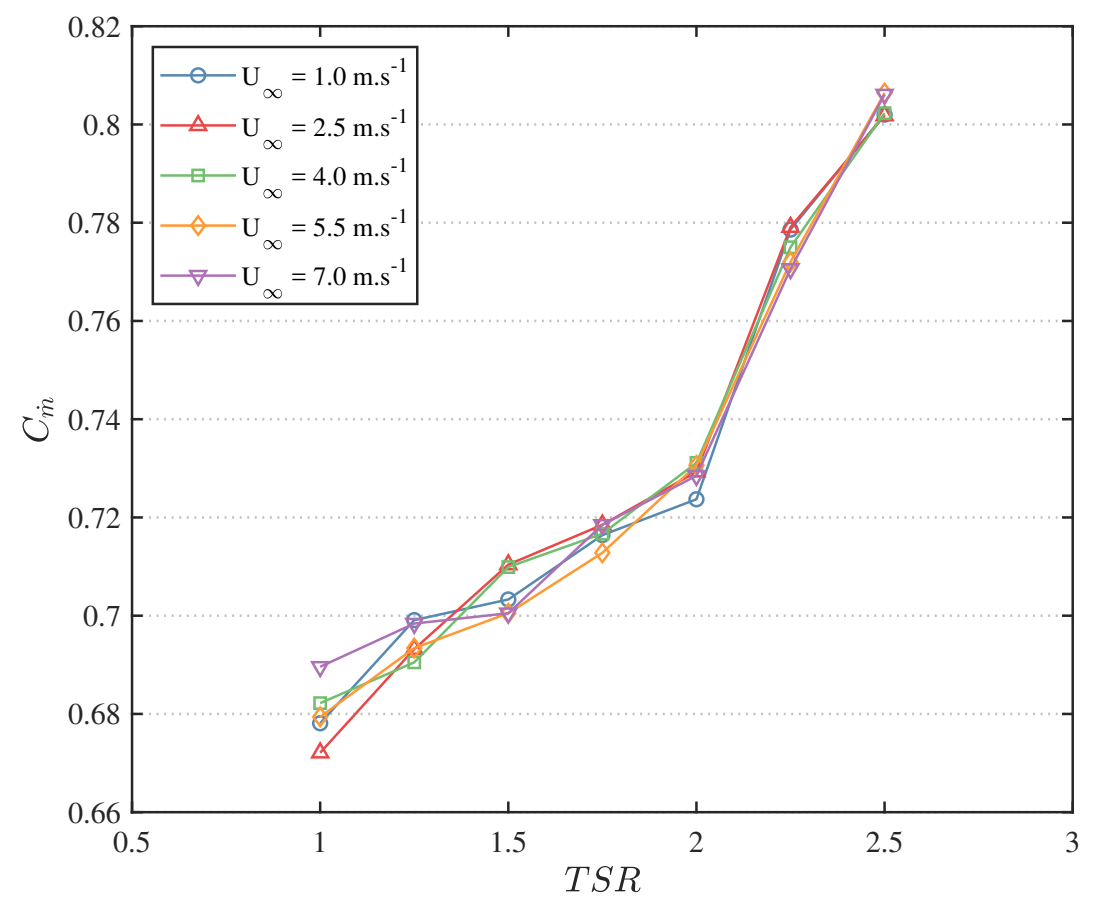

Figure 14: Evaluation of the ducted turbine mass-flow rate coefficient $\left(C_{\dot{m}}\right)$ in relation to TSR

rotor, illustrated in Figure 15a, both of which towards regions of lower pressure. Physically, along the perimeter of the duct, the point at which this occurs is the point of pressure stagnation, where static pressure is the highest, as velocity is nullified, and hence, induces significant drag; in this system, the stagnation point is induced, on average, at $\sim 0.94 R_{d c t}$.

\subsection{Static Pressure on Duct Ridges}

The fluid upstream of the stagnation point induces a unique phenomenon, due to the flow being forced back up the duct, rather than through the turbine. Once the flow surpasses the duct ridges, it interacts with the free-stream; due to the distinct velocities of the two flows, this induces a resultant, deflected flow misaligned to the free-stream, and hence, misaligned to the surface of the duct, resembling flow spillage. Similar to flow around a geometrical bend, substantial flow separation is induced, resulting in a region of re-circulating fluid, known as a separation bubble, within which rotational flow and turbulent mixing occur upon the duct surface, creating a low pressure zone. This concept is illustrated in Figure 15b, where the static pressure along the inner upstream duct surface is pressurised due to the inlet flux. Flux escapes upstream of the stagnation point, and results in a consistent measure of negative static pressure induced along the entire length of the outer duct, at all TSRs, implementing a degree of suction, induced by the re-circulating zone.

This phenomenon occurs at all TSRs modelled, yet the physical size of the re-circulation zone is seen to vary in an inversely proportional manner to the TSR of the rotor. As a result of the substantial static pressure within the duct at low TSRs, due to the lower mass-flow rate 
through the rotor, the flux of the fluid diverted upstream of the rotor is higher than that at higher TSRs. For this reason, the velocity resultant at the interaction with the free-stream is at a more obtuse angle to the surface of the duct than that at higher TSRs, creating a larger vortex zone. In addition, along the surface of the outer duct, the skin friction coefficient is minute, hence depicting that the re-circulation zone is incapable of completely reattaching. In consequence, the re-circulation zone is physically unstable, ensuing in the shedding of the vortices generated within. Therefore, the external flow interacting with the duct will instigate vorticial rotational motion via the region of re-circulating fluid along the length of the duct, which is then shed at the duct outlet.

\subsection{Vortex Shedding}

Aft of the rotor, within the duct, the downstream turbine wake does not expand concomitant to the variation in the duct outlet geometrical profile, yet expands at an angle more acute than the gradient of the inner duct contour. This signifies that the flow refrains from attachment when flowing towards the outlet rim, hence providing evidence of boundary layer separation, decreasing the duct efficiency. In addition, slight flow separation is induced within the fluid region between the wake and the duct due to the free shear layer at the boundaries of the rotor wake, as illustrated in Figure 16. The distinct vortices, formed by the re-circulating fluid region along the circumference of the duct, and shed at the duct outlet as ring vortices, as illustrated in Figure 13, are transported within the turbine wake from this location. At low TSRs, the wake demonstrates a highly disorganised flow structure with turbulent intermixing, as illustrated in Figure 17. The large volume of the re-circulating zone induces substantial rotational motion within the created vortices, resulting in vortex shedding at comparatively high rotational kinetic energy; the high-energy vortices commingle with the low-energy wake, creating a chaotic wake. With an increase in TSR to nominal TSR, a consistent ring vortex stream is identified in the wake. The vortex effect diminishes with an increase in TSR, due to the reduction of the re-circulating fluid zone and, hence, rotational kinetic energy. As the wake contains a higher degree of energy, this results in a more streamline wake at high TSRs; flow irregularity is nonetheless present in the wake, yet is comparatively minute, and is solely transported within until dissipation.

Correspondent to the vortex shedding, an analysis regarding the vortex effect on the axial drag force developed was considered by superimposing a sinusoidal function upon the global drag of the system in an effort to attain the frequency of the fluctuation. As a result, the vortex shedding frequency was found to be linearly proportional to the free-stream velocity, and, hence, Reynolds number $\left(R e_{L}\right)$, as illustrated in Figure 18, in agreement with Strouhal's law; the shedding frequency is, therefore, solely dependent on the free-stream velocity of the domain. The quantity of the vortices shed per rotor rotation, however, act in an opposite manner, being independent of the free-stream velocity, yet dependent on the TSR. Within that attribute, a mean of 2.15 and 0.83 vortices per rotation are shed at low TSR and high TSR, respectively. In attaining a Strouhal number $(S t)$ for the system, the duct length $\left(L_{d c t}\right)$ was utilised as the Strouhal characteristic length $\left(L_{S t}\right)$, hence resulting in a constant mean global Strouhal number of 0.558 for the ducted turbine system. For this reason, vortex shedding may have a significant influence on the system, where the potential 


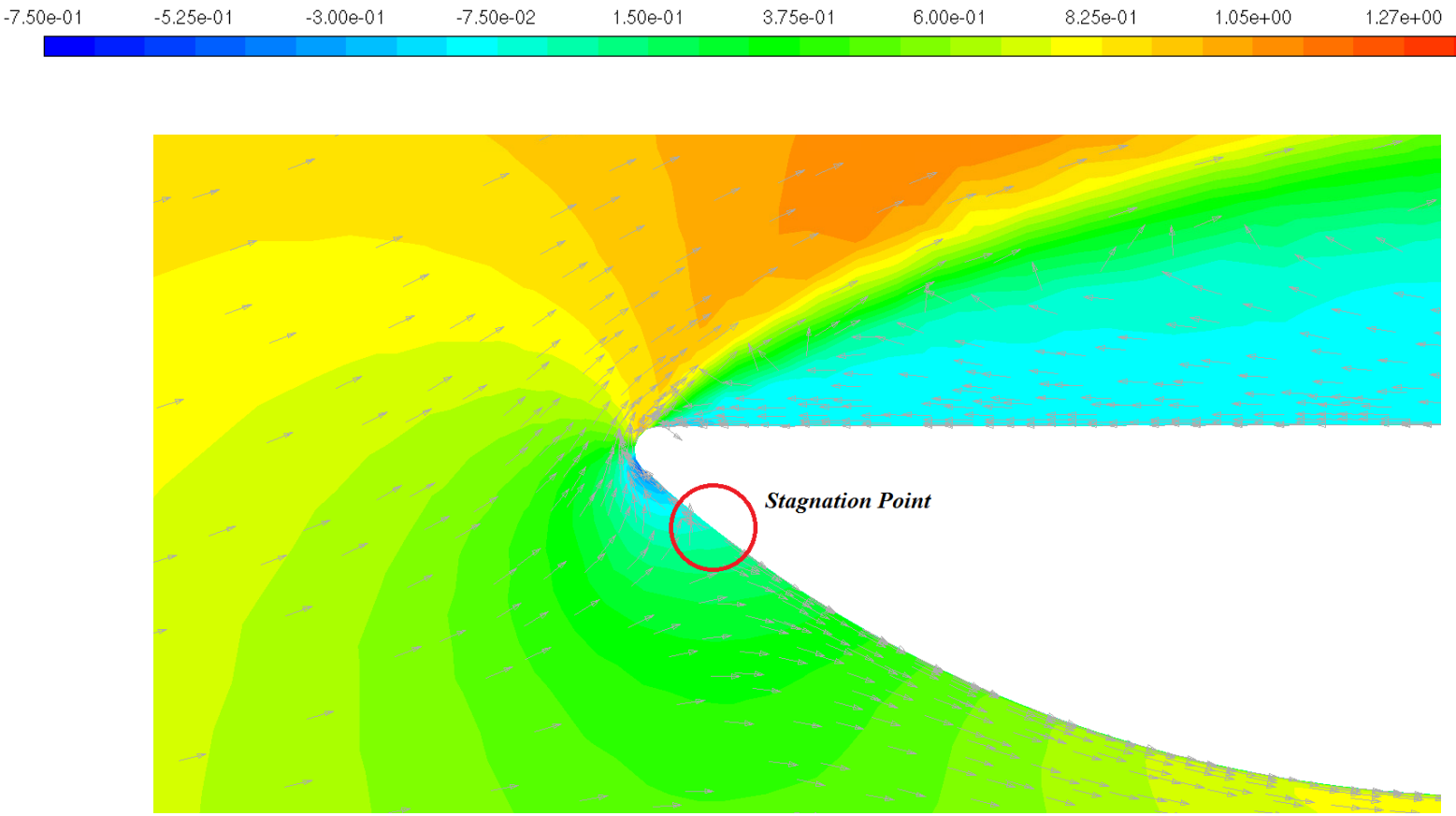

(a) Axial velocity coefficient $\left(\bar{u}_{z}^{*}\right)$ illustration of stagnation point on inner curved duct surface, and re-circulation zone on outer straight duct surface; $T S R=1.75$

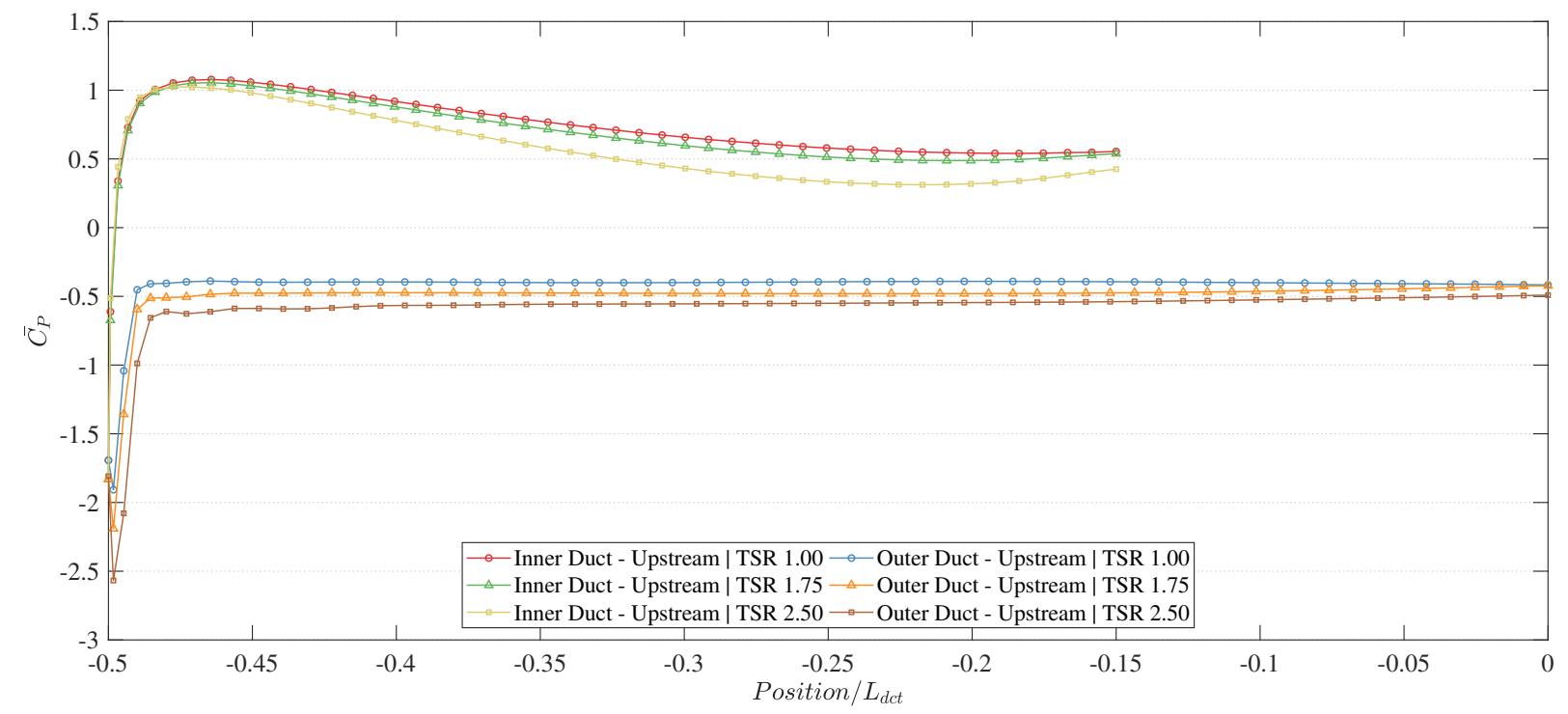

(b) Pressure coefficient $\left(C_{P r}\right)$ values at the inner curved and outer straight duct sections upstream of the rotor $\left(-0.5 \leqslant \frac{z}{L_{d c t}} \leqslant 0\right)$

Figure 15: Representation of the dynamics at the inner curved and outer straight geometry of the duct inlet; $U_{\infty}=4 \mathrm{~m} / \mathrm{s}$

presence of fatigue-inducing structural phenomena, such as vortex-induced vibrations (VIVs) 


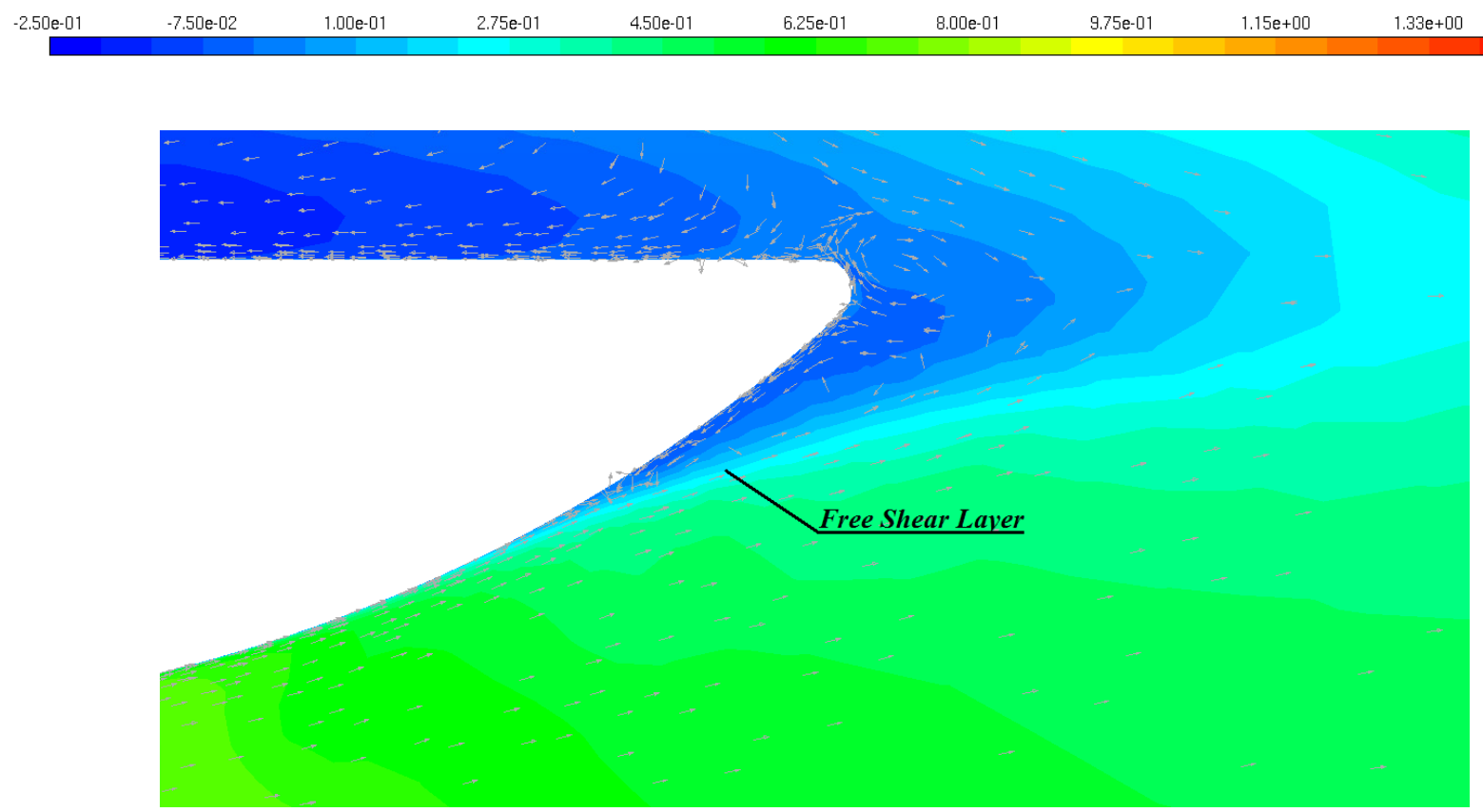

Figure 16: Axial velocity coefficient $\left(\bar{u}_{z}^{*}\right)$ illustration of the free shear layer at the duct outlet; $U_{\infty}=$ $4 \mathrm{~m} / \mathrm{s}, \mathrm{TSR}=1.75$

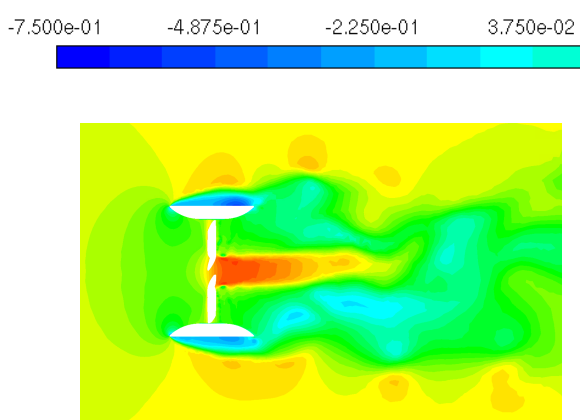

$0.5 T_{\omega}$

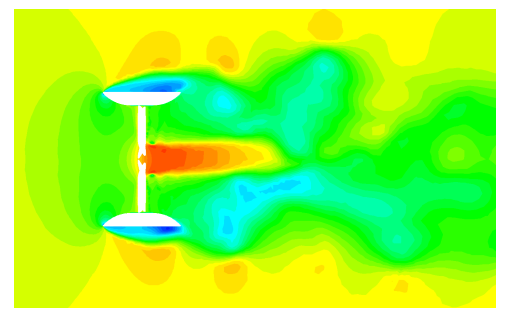

$1 T_{\omega}$

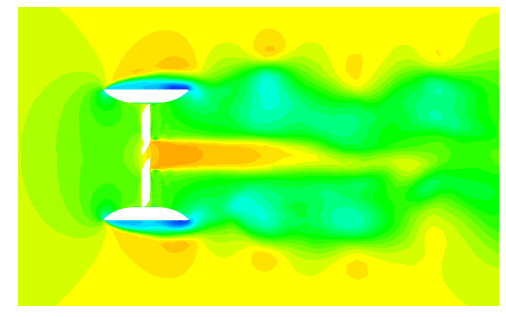

$0.5 T_{\omega}$

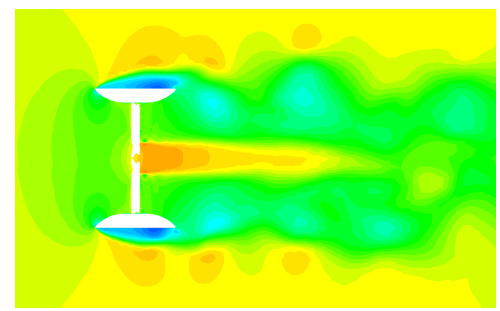

$1 T_{\omega}$

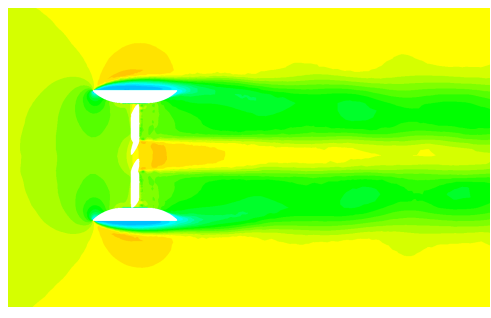

$0.5 T_{\omega}$

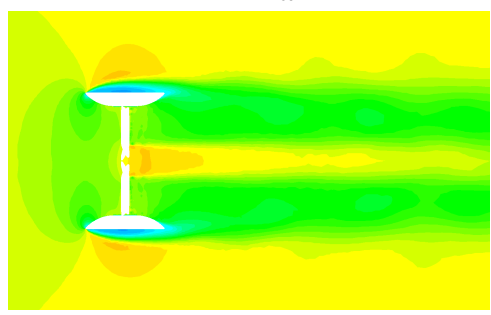

$1 T_{\omega}$
(a) $T S R=1.00$
(b) $T S R=1.75$
(c) $T S R=2.50$

Figure 17: Illustrative top-view representation of the axial velocity coefficient $\left(\bar{u}_{z}^{*}\right)$ within the turbine domain at low, nominal, and high $T S R ; U_{\infty}=4 \mathrm{~m} / \mathrm{s}$ 


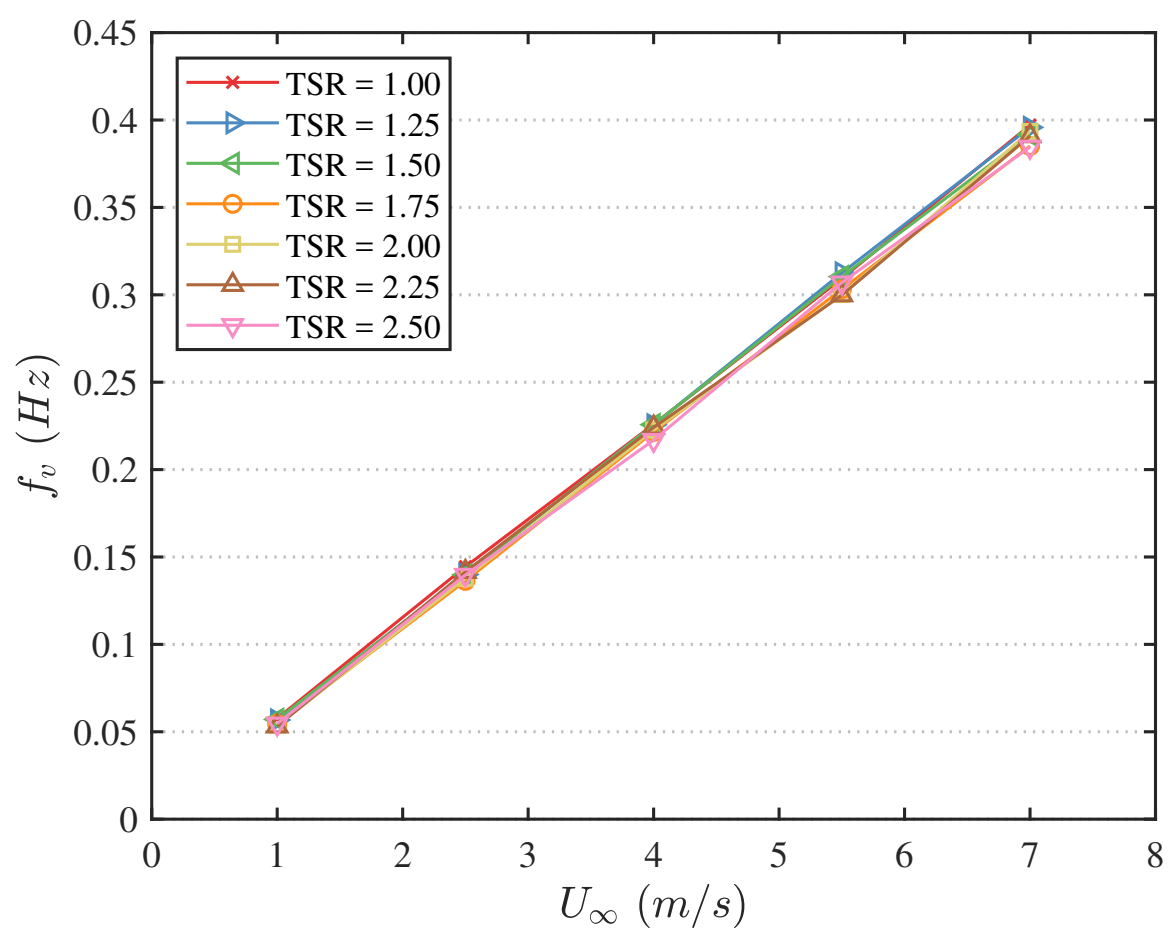

Figure 18: Evaluation of the vortex shedding frequency $\left(f_{v}\right)$ in relation to free-stream velocity $\left(U_{\infty}\right)$ at distinct TSRs

on its foundation, together with fluttering at the blades, become a consequential factor to the dynamics of the system.

\subsection{Blade Analysis}

Further to analysing the static pressure on the duct, in effort of acknowledging the hydrodynamic impact on the blades, the static pressure on distinct sections along the radius of the rotor was considered. Figure 19 illustrates the variation in pressure coefficient at nominal TSR in five physical iterations on the blade, from near-tip $\left(r_{r t r} / R_{r t r}=0.3633\right)$ to near-duct $\left(r_{r t r} / R_{r t r}=0.9300\right)$, to quantitatively extract the static pressure distribution along the rotor radius.

The distribution of static pressure along each section of the blades was found to be largely consistent with each other. With a pressurised side on the upstream surface of the blade, the stagnation point, at higher radii, is precisely at the leading edge of the blade, which shifts inwards along the pressurised surface at lower radii due to the variation in angle-of-attack. Attributable to the profile of the blade, a flat-plate with rounded edges, the acceleration of the flow around the leading edge induces a separation point at the downstream (suction) surface. Separation occurs at the point where the rounded edge joins the flat edges. This aspect is attributable to flat-plates with rounded edges; along the upstream (pressurised) surface, a pressure depression is induced, yet is sustained as a result of the external flow from the inlet of the duct. 


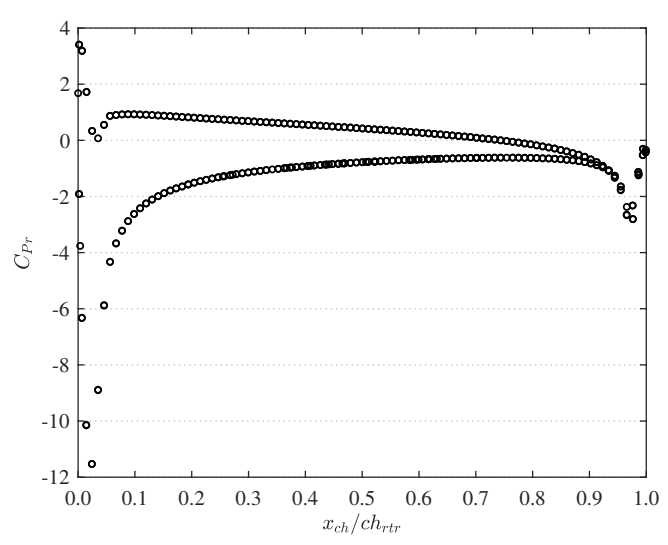

(a) $r_{r t r} / R_{r t r}=0.9300$

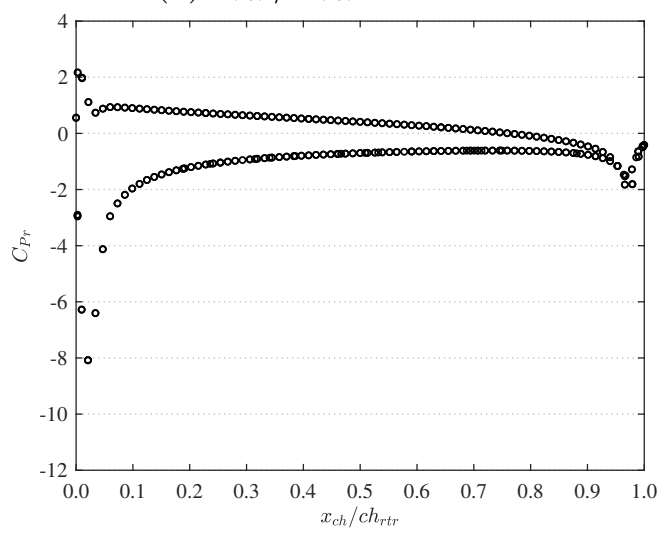

(c) $r_{r t r} / R_{r t r}=0.6466$

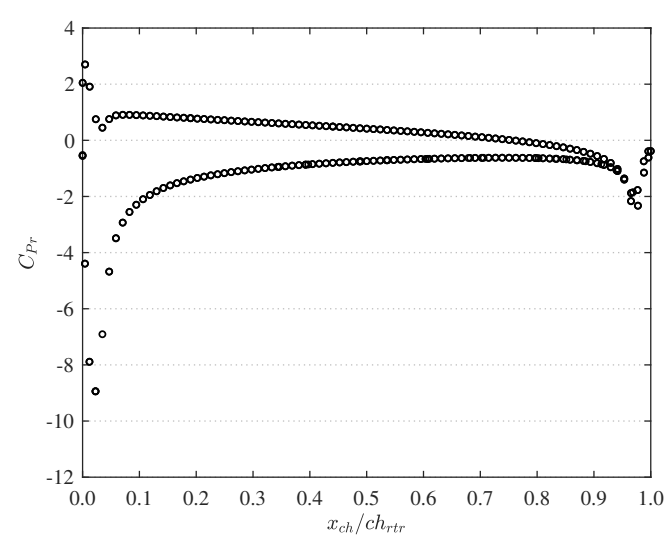

(b) $r_{r t r} / R_{r t r}=0.7600$

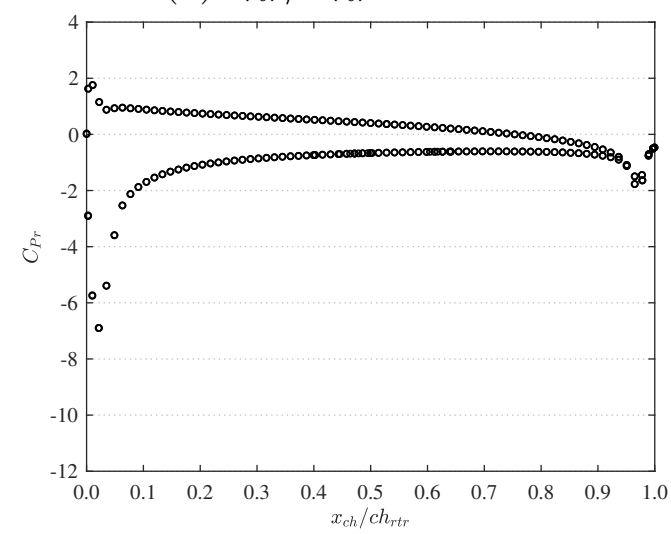

(d) $r_{r t r} / R_{r t r}=0.5333$

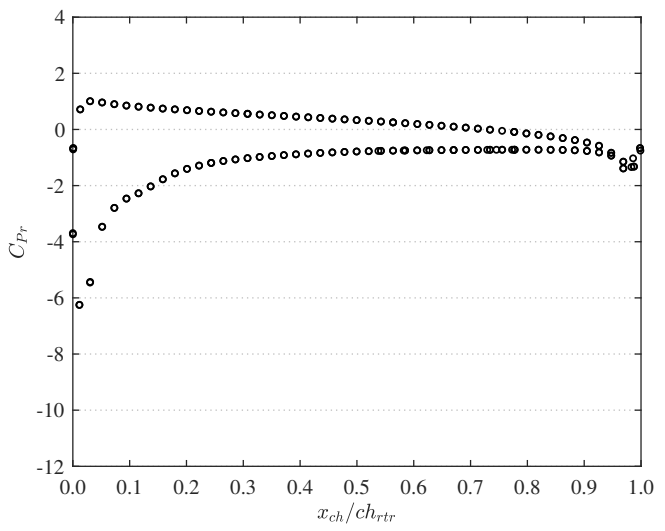

(e) $r_{r t r} / R_{r t r}=0.3633$

Figure 19: Plots of pressure coefficient $\left(C_{P r}\right)$ along the blade chord ratio $\left(x_{c h} / c h_{r t r}\right)$ at distinct rotor radius ratios $\left(r_{r t r} / R_{r t r}\right)$ at $U_{\infty}=4.0 \mathrm{~m} / \mathrm{s}, T S R=1.75$

Suction is dominant throughout the surfaces of the blades, withal at the upstream pressurised surface, where pressure decreases along the chord length, accentuating the inefficacy of the blade profile. A secondary pair of separation points are induced at the locations where the trailing rounded edge joins the flat plate, due to the sudden change in geometry, and 

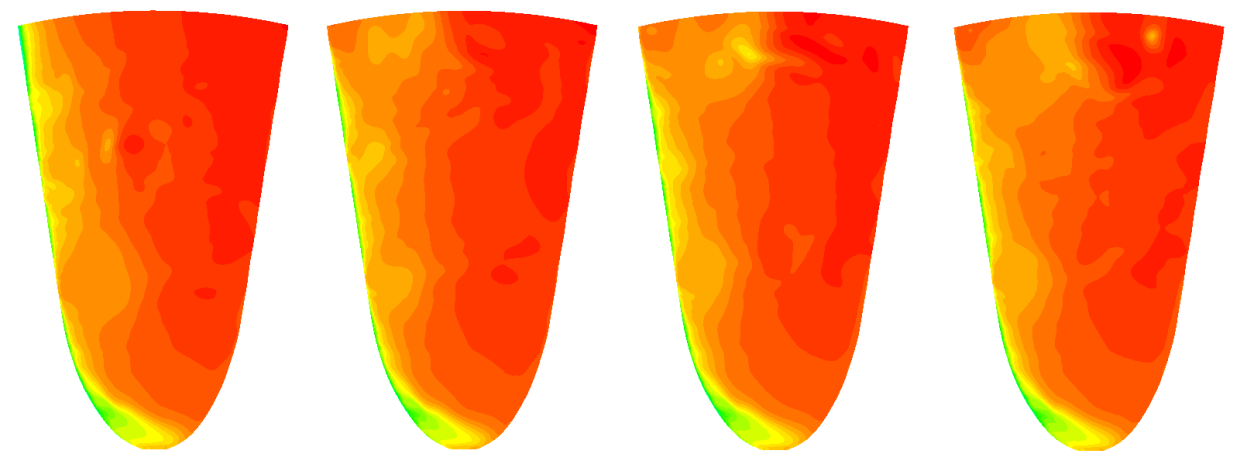

(a) $T S R=1.00$
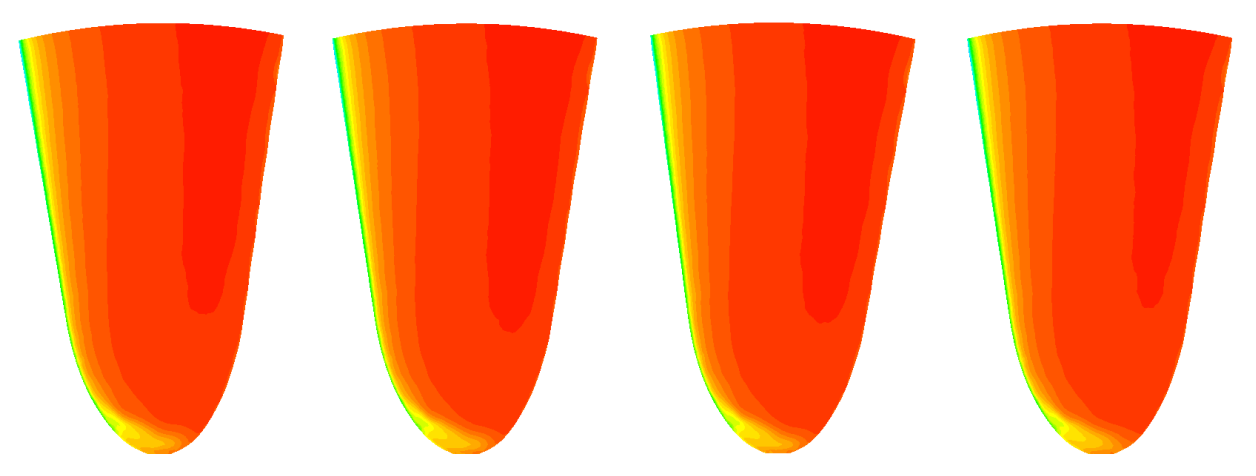

(b) $T S R=1.50$

Figure 20: Pressure coefficient $\left(C_{P r}\right)$ contour representation along the suction edge of the blade at rotation period $\frac{1}{4} T_{\omega}$ (left), $\frac{2}{4} T_{\omega}$ (mid-left), $\frac{3}{4} T_{\omega}$ (mid-right), and $\frac{4}{4} T_{\omega}$ (right) at $U_{\infty}=1.00 \mathrm{~m} / \mathrm{s}$ at low (top) and nominal (bottom) $T S R$

hence, pressure. Precisely at the trailing edge, the suction is substantial enough to engulf the post-separation low-pressure flow towards the wall, increasing the static pressure at this location. This description is consistent at differing radii, yet the extremities decrease proportionally to the radius-position of the blade, primarily due to the comparatively diminished dynamics towards the central axis of the rotor.

In addition to the quantitative measure of static pressure, in an effort to further contemplate the flow dynamics at the blade surfaces, pressure contours along the downstream suction side, illustrated in Figures 20 and 21, were qualitatively attained at low and nominal TSRs at quarterly positions of rotational period $\left(T_{\omega}\right)$ for low and mid free-streams. Regions of stall are elucidated at low rotational velocities, which recede with an increase in TSR; this accompanies well the analysis and speculations put forward as a result of the variation in angle of attack and resultant induced lift. By means of these contours, distinct aspects of 

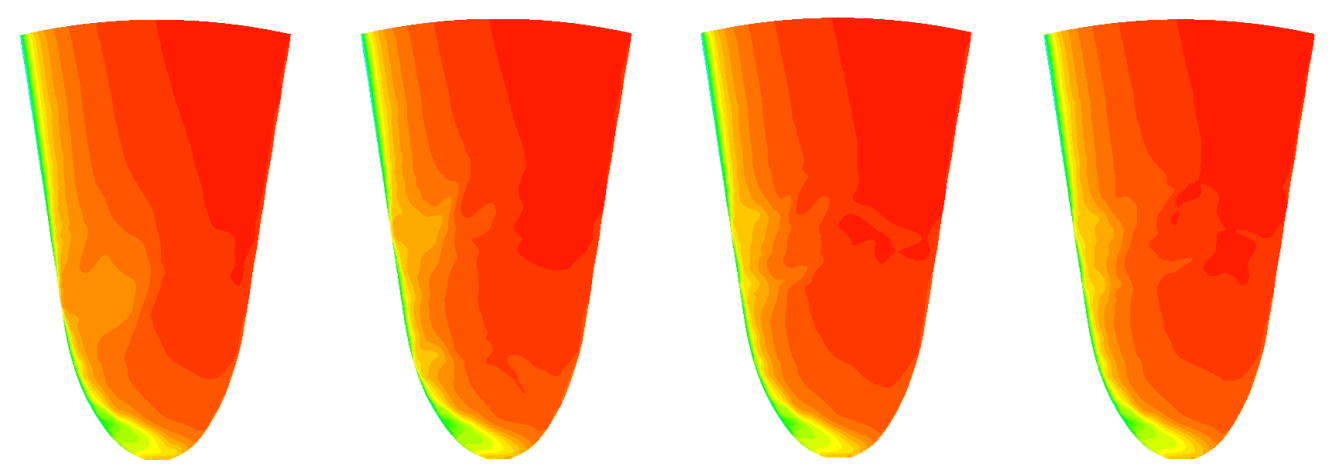

(a) $T S R=1.00$
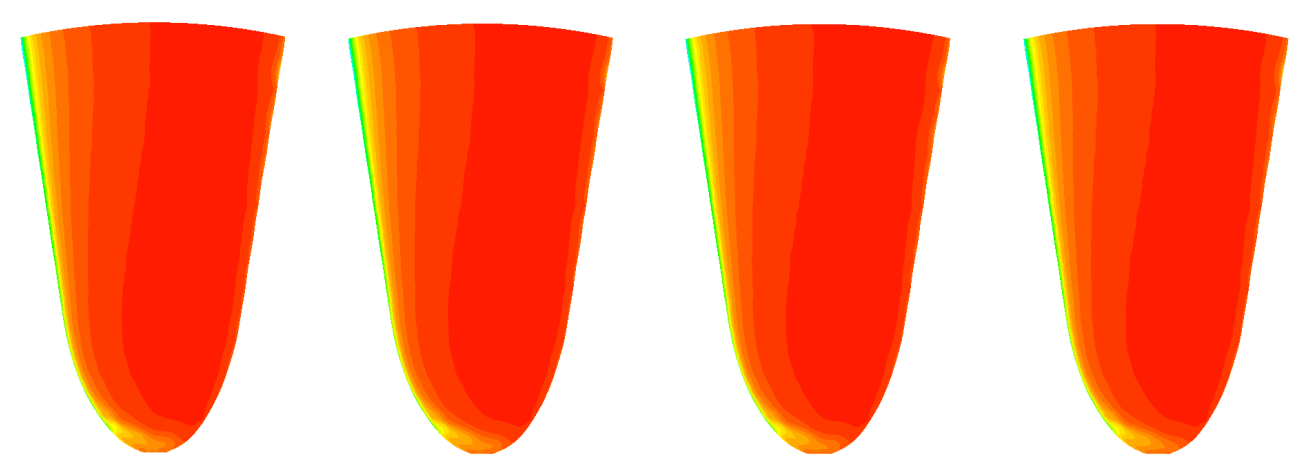

(b) $T S R=1.75$

Figure 21: Pressure coefficient $\left(C_{P r}\right)$ contour representation along the suction edge of the blade at rotation period $\frac{1}{4} T_{\omega}$ (left), $\frac{2}{4} T_{\omega}$ (mid-left), $\frac{3}{4} T_{\omega}$ (mid-right), and $\frac{4}{4} T_{\omega}$ (right) at $U_{\infty}=4.00 \mathrm{~m} / \mathrm{s}$ at low (top) and nominal (bottom) TSR

the flow are established, specifically the spanwise flow acting along the blade, ascertaining the requirement of three-dimensionality when analysing rotating flows.

\subsection{Wake Velocity Profiles}

A potential aspect of the installation of ducts is the constrainment of the downstream wake; an advantage in the effort to limit wake interaction with subsequent rotors within a turbine farm. The axial velocity profiles of the wake of the rotor, from $0.5 D_{r t r}$ to $6 D_{r t r}$ downstream, are uniquely compound as a result of this installation. Illustrated in Figure 22, the dimensionless axial velocity $\left(\bar{u}_{z}^{*}=\frac{\bar{u}_{z}}{U_{\infty}}\right)$ profiles at the distinct free-stream velocities considered are largely identical.

Within the near-wake, at $0.5 D_{r t r}$, the maximum axial wake velocity is identified to be at the centre; a result of the open-centre of the turbine. This velocity surpasses the value of the 


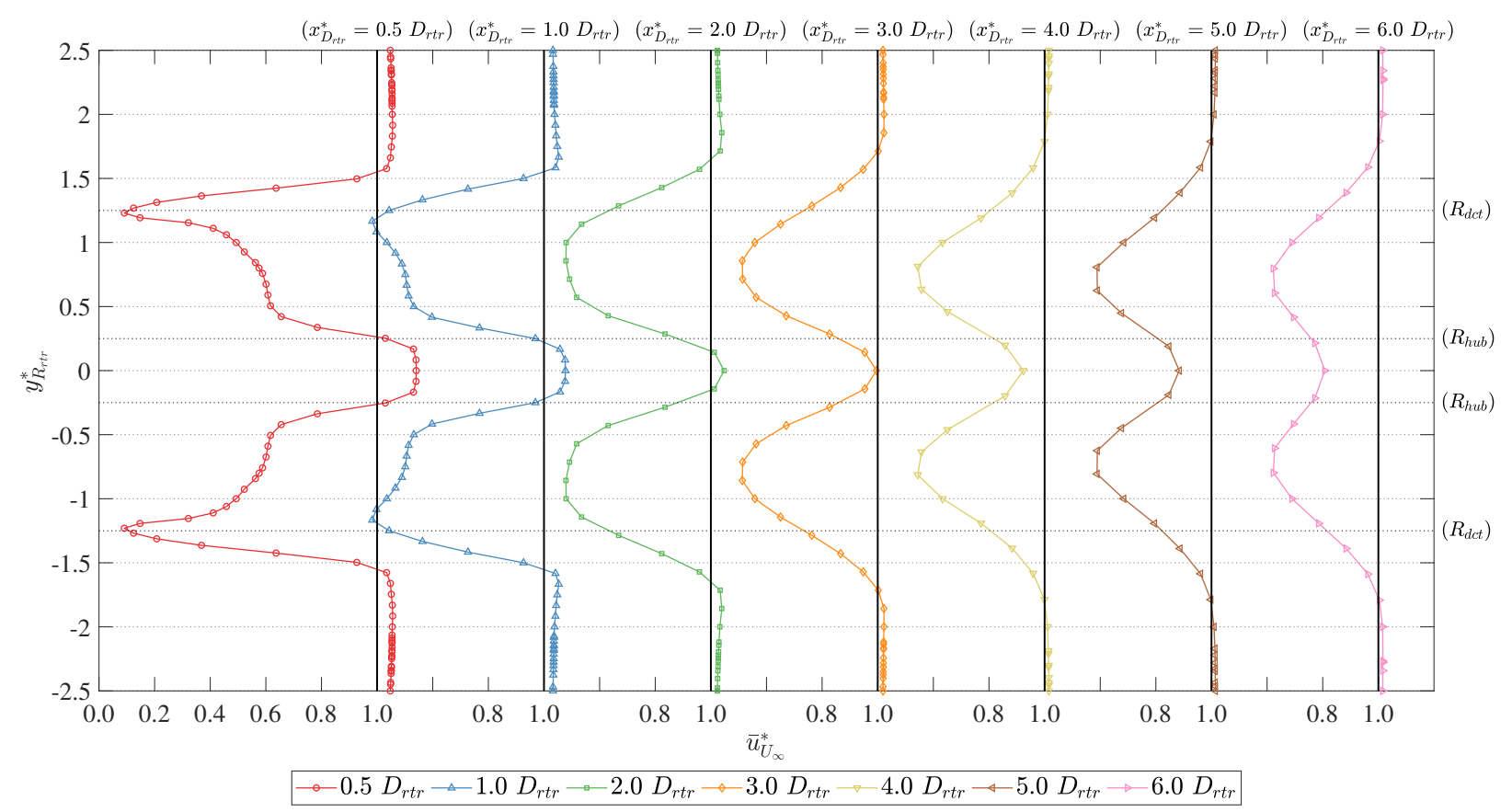

Figure 22: Dimensionless axial velocity $\left(\bar{u}_{z}^{*}\right)$ profiles within the ducted turbine wake at different rotor diameter displacements downstream $-U_{\infty}=4 \mathrm{~m} / \mathrm{s}, T S R=1.75$

free-stream magnitude, acknowledging a phenomenon typically established in flow through an orifice: an induction of flow acceleration. This acceleration comes about in consequence of the substantial static pressure retained within the duct immediately upstream of the rotor, forcing fluid out of the open-centre. This outcome sacrifices torque on the blades, as a degree of fluid at the wall flows in a radial direction towards the orifice at the blade tip, rather than in a tangential orientation along the blades. In continuation, the axial velocity diminishes along the radius of the turbine $\left(y_{R_{r t r}}^{*}=\frac{y}{R_{r t r}}\right)$ due to the presence of the blades and duct, where the point of minima axial velocity is situated at the ridge of the duct. The axial velocity then increases rapidly to a magnitude above the free-stream, an effect from the flow acceleration at the upstream ridge, within a displacement of $1.5 R_{r t r}$ (or $1.2 R_{d c t}$ ) from the central axis of the turbine, portraying the capacity of a duct in restricting the extent of the wake growth. The velocity then decreases gradually to the free-stream magnitude.

As the wake flows further downstream, regions of higher kinetic energy dissipate to regions of lower kinetic energy, hence axial velocity decreases within the region in the vicinity of the orifice to supplement the regions in the vicinity of the duct. The velocity profiles become more rounded, signifying an efficient distribution of energy. Far wake, at $6 D_{r t r}$, the velocity profile has largely regained its energy and becomes more consistent along the turbine radius, albeit portraying its unique aspects, particularly at orifice and duct regions. 


\section{Conclusion}

This study put forward an investigation into the hydrodynamic performance concerning a ducted, high-solidity tidal turbine utilising blade-resolved computational fluid dynamics. The research strived to overcome the limitations of actuator disc and blade-element momentum theory methodologies by implementing a computational fluid dynamic analysis with blade-explicit actuality, coupled with general continuity at both the domain and turbine. This feature allows the aspect of modelling flow three-dimensionality at the turbine, together with induced turbulence effects, in further effort of numerically replicating its true performance. Validated by means of a direct comparison to experimentation in numerically replicating the physics of rotating blades, the numerical outcomes of the main performance factors of the full-scale ducted turbine, portraying a peak power coefficient of 0.34 with a thrust coefficient of 1.00 at a nominal tip-speed ratio of 1.75 , were ascertained to justify the applicability of the full-scale analysis and condone further fluid dynamic analysis, namely the hydrodynamic effects along the duct, mass-flow rate through the duct, vortex shedding within the domain, static pressure along the rotor blades, and velocity profiles within the rotor wake.

In summary of the numerical analysis of a ducted high-solidity tidal turbine, it has been observed that, due to the incorporation of a duct with a high number of blades, and hence blade area, the phenomena induced by the fluid-structure interaction are substantial. In effect of the force equilibrium between the magnitude of the flow interaction at the upstream surfaces of the blades with that at the downstream blade surfaces, the resultant torque is substantial at low TSRs, yet decreases rapidly with an increase in TSR. This short-spanning range in TSR is, hence, a direct attribute of the high-solidity properties of the turbine, and is, therefore, the primal reason of its incapability to acquire high values of mechanical power, albeit the high torque attained. This outcome agrees well with Betz's projections for "medium-speed windmills".

The drag on the rotor was found to decrease significantly with TSR, mainly due to the decreasing static pressure difference at higher rotational to free-stream velocity ratios, in consequence of higher mass-flow rates. The most significant phenomena, however, is induced as a result of the introduction of the duct. A stagnant pressure point is developed along the duct inlet, where fluid upstream of this point is forced out of the inlet to flow along the outer flat surface of the duct. Due to its interaction with the free-stream at the duct ridge, a region of low pressure is created, inducing a re-circulating fluid zone. In effect, along the length of the duct, the static pressure at the flat outer surface of the duct is significantly low, inducing suction. Ring vortices are generated as a result of the re-circulating zone, and are shed at the duct outlet. The vortices commingle with the rotor wake, prompting chaotic flow at low TSRs, which streamlines at higher TSRs.

Having validated the numerical model for rotating bladed systems by means of a direct comparison to experimentation in literature on terms of three distinct parameters, notably power coefficient, thrust coefficient, and velocity profiles in the near and far wake, together with comparison of the full-scale analysis to literature and a secondary numerical methodology, the concluded outcomes are assumed to hold. Albeit, the turbulence model utilised in 
this work has been documented to be oversensitive, and hence, may overestimate physical parameters generated at the wall.

Notwithstanding, this work provides a plausible analysis in evidence to the notion that ducted turbines generate higher power per rotor unit area. This is due to the flow being converged towards the rotor when impacting the inner duct. In elaboration of these claims, the power generated by a ducted rotor would be approximately equal to the power generated by an unducted rotor $\left(P_{r t r_{d c t d}} \approx P_{r t r_{u n d c t d}}\right)$, given that the radius of the duct is equal to the radius of the unducted rotor $\left(R_{d c t}=R_{\text {rtr } r_{\text {undctd }}}\right)$, where the true cut-off area is the region of stagnant pressure on the duct inlet geometry, which is dependent on the cross-sectional formation. Under this prospect, the power per unit rotor area of a ducted rotor would be higher in comparison to the power per unit rotor area of an unducted turbine as the area of the ducted rotor is significantly lesser than that of the unducted rotor $\left(A_{r t r_{d c t d}} \ll A_{r t r_{u n d c t d}}\right)$. Albeit the potential increase in power per rotor unit area, the thrust coefficient, in addition to the complementary phenomena, induced as a result of the implementation of the duct, was found to be physically substantial, and hence, considerably negates any related advantageous aspects of the installation.

In essence, as a result of the induced hydrodynamic Venturi effect, it may be speculated that if the areal annulus in which the duct coincides is lower in both capital and operation cost than the blades present in an identical areal annulus of an unducted turbine, then potentially-sustainable advantages in relation to an implementation of a duct may be present. Albeit this indication, this study solely considers a single geometrical design of a duct within a constant flow in open-water conditions, which brings about a degree of assumptions in comparison to real-world implementations, namely absences of variations in flow direction, differences in inlet turbulence intensities and turbulent length scales, and external inlet shear flow, potentially inducing a distinction between the lift force induced at regions along the duct, developing a resultant lift force, requiring further analysis on its foundational supports.

\section{Acknowledgments}

The research work disclosed in this publication is partially funded by the Endeavour Scholarship Scheme (Malta). Scholarships are part-financed by the European Union - European Social Fund (ESF) - Operational Programme II - Cohesion Policy 2014-2020: "Investing in human capital to create more opportunities and promote the well-being of society".

Results were obtained using ARCHIE-WeSt High Performance Computer (www.archiewest.ac.uk). 


\section{References}

[1] F. O Rourke, F. Boyle, and A. Reynolds, "Tidal energy update 2009," Applied Energy, vol. 87, no. 2, pp. 398-409, 2010.

[2] F. M. White, "Fluid Mechanics," in Fluid Mechanics, ch. 6, pp. 325-426, New York: McGraw Hill, 8th ed., 2015.

[3] S. C. Allsop, Hydrodynamic Modelling for Structural Analysis of Tidal Stream Turbine Blades. PhD thesis, University of Edinburgh, 62018.

[4] S. Allsop, C. Peyrard, P. R. Thies, E. Boulougouris, and G. P. Harrison, "Hydrodynamic analysis of a ducted, open centre tidal stream turbine using blade element momentum theory," Ocean Engineering, vol. 141, pp. 531-542, 92017.

[5] C. Belloni, R. Willden, and G. Houlsby, "An investigation of ducted and open-centre tidal turbines employing CFD-embedded BEM," Renewable Energy, vol. 108, pp. 622-634, 82017.

[6] A. Betz, "Windmills in the light of modern research," tech. rep., National Advisory Committee for Aeronautics, Washington, DC, 81928.

[7] P. Gipe, "WIND-WORKS: Ducted Wind Turbines," 2018.

[8] Bloomberg L.P., "OpenHydro Group Limited: Private Company Information - Bloomberg," 2018.

[9] OpenHydro Group Ltd., "Projects," 2016.

[10] ReNews, "Atlantis-to-decommission-seagen - reNews - Renewable Energy News," 2016.

[11] The Canadian Press, "Cape Sharp Tidal turbine in Bay of Fundy now being monitored remotely," 2018.

[12] E. Benezet, "La grande vague des hydroliennes," 2018.

[13] D. G. Phillips, An investigation on diffuser augmented wind turbine design. PhD thesis, The University of Auckland, 2003.

[14] D. G. Phillips, P. J. Richards, and R. G. J. Flay, "DIFFUSER DEVELOPMENT FOR A DIFFUSER AUGMENTED WIND TURBINE USING COMPUTATIONAL FLUID DYNAMICS," tech. rep., The University of Auckland, Auckland, New Zealand, 2005.

[15] K. D. Visser, "Wind Tamer Turbine Performance Report 1kW-15 kW Performance Predictions," tech. rep., Clarkson University, Potsdam, 2009.

[16] M. O. L. Hansen, N. N. Sørensen, and R. G. J. Flay, "Effect of Placing a Diffuser around a Wind Turbine," Wind Energy, vol. 3, pp. 207-213, 102000.

[17] M. J. Werle and W. M. Presz, "Ducted Wind/Water Turbines and Propellers Revisited," Journal of Propulsion and Power, vol. 24, pp. 1146-1150, 92008.

[18] H. Grassmann, F. Bet, G. Cabras, M. Ceschia, D. Cobai, and C. Delpapa, "A partially static turbinefirst experimental results," Renewable Energy, vol. 28, pp. 1779-1785, 2003.

[19] H. Grassmann, F. Bet, M. Ceschia, and M. Ganis, "On the physics of partially static turbines," Renewable Energy, vol. 29, pp. 491-499, 42004.

[20] Y. Ohya, T. Karasudani, A. Sakurai, K.-i. Abe, and M. Inoue, "Development of a shrouded wind turbine with a flanged diffuser," Journal of Wind Engineering and Industrial Aerodynamics, vol. 96, pp. 524-539, 52008.

[21] C. Munch, M. Vonlanthen, J. Gomes, R. Luquet, P. Guinard, and F. Avellan, "Design and Performance Assessment of a Tidal Ducted Turbine," in 3rd IAHR International Meeting of the Workgroup on Cavitation and Dynamic Problems in Hydraulic Machinery and Systems, (Brno, Czech Republic), 2009.

[22] D. L. Gaden and E. L. Bibeau, "A numerical investigation into the effect of diffusers on the performance of hydro kinetic turbines using a validated momentum source turbine model," Renewable Energy, vol. 35, pp. 1152-1158, 62010.

[23] M. Shives, C. Crawford, and S. Grovue, "A tuned actuator cylinder approach for predicting crossflow turbine performance with wake interaction and channel blockage effects," International Journal of Marine Energy, vol. 18, pp. 30-56, 62017.

[24] U. Ahmed, D. Apsley, I. Afgan, T. Stallard, and P. Stansby, "Fluctuating loads on a tidal turbine due to velocity shear and turbulence: Comparison of CFD with field data," Renewable Energy, vol. 112, pp. 235-246, 112017. 
[25] M. A. Holst, O. G. Dahlhaug, and C. Faudot, "CFD Analysis of Wave-Induced Loads on Tidal Turbine Blades," IEEE Journal of Oceanic Engineering, vol. 40, pp. 506-521, 2015.

[26] T. Setoguchi, N. Shiomi, and K. Kaneko, "Development of two-way diffuser for fluid energy conversion system," Renewable Energy, vol. 29, pp. 1757-1771, 82004.

[27] C. F. Fleming and R. H. Willden, "Analysis of bi-directional ducted tidal turbine performance," International Journal of Marine Energy, vol. 16, pp. 162-173, 2016.

[28] C. S. Belloni, R. H. Willden, and G. T. Houlsby, "A Numerical Analysis of Bidirectional Ducted Tidal Turbines in Yawed Flow," Marine Technology Society Journal, vol. 47, pp. 23-35, 72013.

[29] C. Belloni, Hydrodynamics of Ducted and Open-Centre Tidal Turbines. PhD thesis, Oxford University, UK, 2013.

[30] M. G. Borg, Q. Xiao, A. Incecik, S. Allsop, and C. Peyrard, "Numerical Analysis of a Ducted HighSolidity Tidal Turbine," in OCEANS'18 MTS/IEEE Kobe, (Kobe, Japan), IEEE, 2018.

[31] D. C. Wilcox, Turbulence Modeling for CFD, Third Edition. San Diego: DCW Industries, Inc, third ed., 2006.

[32] P. Mycek, B. Gaurier, G. Germain, G. Pinon, and E. Rivoalen, "Renewable Energy Experimental study of the turbulence intensity effects on marine current turbines behaviour. Part I: One single turbine," Renewable Energy, vol. 66, pp. 729-746, 2014.

[33] S. P. Neill, J. R. Jordan, and S. J. Couch, "Impact of tidal energy converter (TEC) arrays on the dynamics of headland sand banks," Renewable Energy, vol. 37, pp. 387-397, 12012.

[34] A. Bahaj and L. Myers, "Analytical estimates of the energy yield potential from the Alderney Race (Channel Islands) using marine current energy converters," Renewable Energy, vol. 29, pp. 1931-1945, 102004.

[35] ANSYS Inc., FLUENT User's Guide. Lebanon, NH: ANSYS Inc., 5 ed., 1998.

[36] T. Kajishima and K. Taira, Computational Fluid Dynamics: Incompressible Turbulent Flows. Cham: Springer International Publishing, 2017. 


\title{
A numerical performance analysis of a ducted, high-solidity tidal turbine
}

\author{
Mitchell G. Borg ${ }^{\mathrm{a}}$, Qing Xiao ${ }^{\mathrm{a}, *}$, Steven Allsop ${ }^{\mathrm{b}}$, Atilla Incecik ${ }^{\mathrm{a}}$, Christophe Peyrard ${ }^{\mathrm{c}}$ \\ ${ }^{a}$ Department of Naval Architecture, Ocean, and Marine Engineering \\ University of Strathclyde \\ Glasgow, Scotland, United Kingdom \\ ${ }^{b}$ Industrial Doctoral Centre for Offshore Renewable Energy (IDCORE) \\ University of Edinburgh \\ Edinburgh, Scotland, United Kingdom \\ ${ }^{c}$ Electricité de France Research and Development \\ $E D F R E D$ \\ Chatou, Ile-de-France, France
}

\begin{abstract}
This study puts forward an investigation into the hydrodynamic performance concerning a ducted high-solidity tidal turbine utilising blade-resolved computational fluid dynamics. The model achieves similarity values of over 0.96 with experimentation data regarding a three-bladed horizontal-axis tidal turbine in validation of three distinct parameters: power \& torque coefficient, thrust coefficient, and wake velocity profiles. Accordingly, the model was employed for the analysis of a ducted, high-solidity turbine in axially-aligned flows at distinct free-stream velocities. The resultant hydrodynamic performance characteristics portrayed a peak power coefficient of 0.34 , with a thrust coefficient of 1.00 , at a nominal tipspeed ratio of 1.75. Coefficient trend agreement was attained between the numerical model and experimentation data established in literature and blade-element momentum theory; the model furthers the analysis by elaborating the temporal hydrodynamic features induced by the fluid-structure interaction in specification to the wake formation velocity profiles, pressure distribution along the blades and duct, volumetric flow rate, and vortex shedding effects to establish the characteristic flow physics of the tidal turbine.

Keywords: turbine performance, high solidity, open centre, tidal turbine, ducted turbine, RSM
\end{abstract}

\section{Introduction}

Efforts to increase the efficiency of energy-generating turbines have been in constant development following their implementation in the global market. At the forefront of the

${ }^{*}$ Corresponding author

Email addresses: mitchell.borg@strath.ac.uk (Mitchell G. Borg), qing.xiao@strath.ac.uk (Qing Xiao), christophe.peyrard@edf.fr (Christophe Peyrard) 
pertinent research is the effort of increasing the mass flow through the turbine, along with the constrainment and alignment of the wake flow, to facilitate further turbine installations [1]. As a result of the research and development attained, two conventional classifications of installations have been implemented around a turbine rotor: unidirectional ducts and bidirectional ducts. Both systems have been considered to enhance the performance of a turbine by increasing the flow velocity along the structure as a result of the induced physical phenomena. Albeit the potential improvements, the performance capabilities of a rotor within a bidirectional duct are inadequately understood.

The physics governing the flow within a vacant duct, in contrast, are widely understood. In accordance to Bernoulli's principle, the energy state of an ideal fluid is a relationship between its kinetic and potential energy, hence a function of its pressure, density, and velocity, through a spatial discrepancy [2]. In consideration of a bidirectional duct with a constricted section, hence a decrease in internal cross-sectional area, a Venturi effect phenomenon is induced through the system. Through this effect, in concordance to the principle of mass continuity, the axial velocity of an incompressible fluid increases along the constriction, along with a decrease in static pressure. In this confine, the application is numerically simplistic if a finite section of a duct system is considered, as the parameters are largely consistent. Nonetheless, an open-ended duct succumb to variable parameters of an external fluid domain, together with the implementation of a rotor, which, as a structure, will vary the pressure and velocity gradients through the duct, will induce high degrees of complexity. The potential increase in flow velocity through the turbine, however, as a result of the Venturi effect, is of particular interest. For this reason, the aim of this research is to analyse the hydrodynamic performance of a ducted high-solidity tidal turbine, particularly within the presence of duct-rotor influence.

Albeit a handful of recent research ventures have tackled the fluid dynamics of the implementation of a duct feature in a turbine system, they have had numerical forms of methodological limitations, such as analytically capturing mass and momentum continuity through the duct [3], or discretising the blade structure into elements [4,5], rather than portraying its true physicality. This research strives to overcome the related limitations by implementing a computational fluid dynamic (CFD) analysis with blade-explicit actuality, coupled with general continuity at both the domain and turbine. This feature allows the aspect of modelling flow three-dimensionality at the turbine, together with induced turbulence effects, in further effort of numerically replicating its true performance. Validated by means of a direct comparison to experimentation in numerically replicating the physics of rotating blades, the numerical outcomes of the main performance factors of the full-scale ducted turbine, in terms of power, torque, and thrust coefficients, were compared to literature [6] and blade-element momentum theory [4] to ascertain the applicability of the full-scale analysis, and justify further fluid dynamic analysis, namely the hydrodynamic effects along the duct, mass-flow rate through the duct, vortex shedding within the domain, static pressure along the rotor blades, and velocity profiles within the rotor wake. This written work is composed of six sections in discourse of: (i) an introduction to the concept of ducted turbines, with an overview of its state-of-the-art, (ii) the methodologies utilised for its numerical analysis, (iii) a characterisation of turbine theory and the modelling schemes utilised, (iv) a confirmation 
of the numerical model validation, (v) a discussion of the results attained, together with (vi) a general conclusion of the analysis.

\subsection{Commercial Application}

A number of commercial endeavours have attempted to adopt ducted turbine technology in achieving economical prospects, yet a substantial quantity of the investments have ensued in insolvency and cessation [7]. Amongst the ventures, OpenHydro Ltd. had, disputably, attained quasi-sustainable implementation with an open-centre ducted design approach in which an aperture is employed at the centre of the turbine in replacement of a turbine nose cone hub $[8,9]$. Due to this approach, the setup not only utilises the duct as a flow modifier, but also as a unit in which the power generating system may be housed.

Tidal stream turbine (TST) technology has been in its primal, developmental phase for the past decade with substantial potential, yet has not taken off as rapidly as had been predicted. This outcome has been argued to be largely due to engineering challenges in designing for extreme operating conditions, in combination with political and environmental factors, which has limited the rate of industry maturity [1]. Amongst the earliest groundbreaking tidal projects, the MCT SeaGen, a 1.2 MW twin-rotor device, was installed within the Strangford Loch, Northern Ireland in 2008, and, subsequent to generating $10 \mathrm{GWh}$ of electrical supply, decommissioned in 2016 [10]. Yet, with very few corresponding projects attaining a similar degree of impact in the renewable energy field, corporate interest has largely diminished within the tidal industry. Albeit the drawbacks observed, European, North American, and Asian nations and unions have persisted their investment in the deployment of full-scale tidal turbine arrays, with a wide range of the European investment being installed within territorial waters of France and the United Kingdom.

A profusion of turbine designs have undergone hydrodynamic investigations for tidal applications, yet two configurations have been largely integrated within the industry, both of which having attained throughput to commercialisation deployment scales. Characteristic to the aerodynamic equivalent, three-bladed horizontal axis tidal turbine (HATT) designs have been commissioned in large-scale installations. Having successfully installed four HATTs in its primal stages, Phase 1A, the MeyGen project is amongst the more notable commercial efforts in HATT energy generation. Future prospects include Phases $1 \mathrm{~B}$ and $1 \mathrm{C}$, potentially introducing an additional $6 \mathrm{MW}$ (four turbines) and 73.5 MW (forty-nine turbines) respectively to the grid, together with Phases 2 and 3 currently within planning stages. In continuation, the ducted turbine configuration has enticed interest due to the potential increase in power extraction as a result of the enhancement of mass flow through the rotor, in addition to benefits as: alignment of axial velocity in yawed flows, providing housing for direct-drive rim generators, and elimination of subsidiary mechanical systems and components, such as gearboxes. DCNS/OpenHydro Ltd. had successfully installed a $2 \mathrm{MW}$ turbine in the Bay of Fundy, Canada, portrayed in Figure 1a, together with a pair of 500 $\mathrm{kW}$ rated capacity turbines, illustrated in Figure 1b, as a demonstration array in PaimpolBréhat, Northern France, in collaboration with EDF France. Yet, despite the milestones, the imminent economic instability of the development of ducted turbines may potentially falter the perception of this technology. 


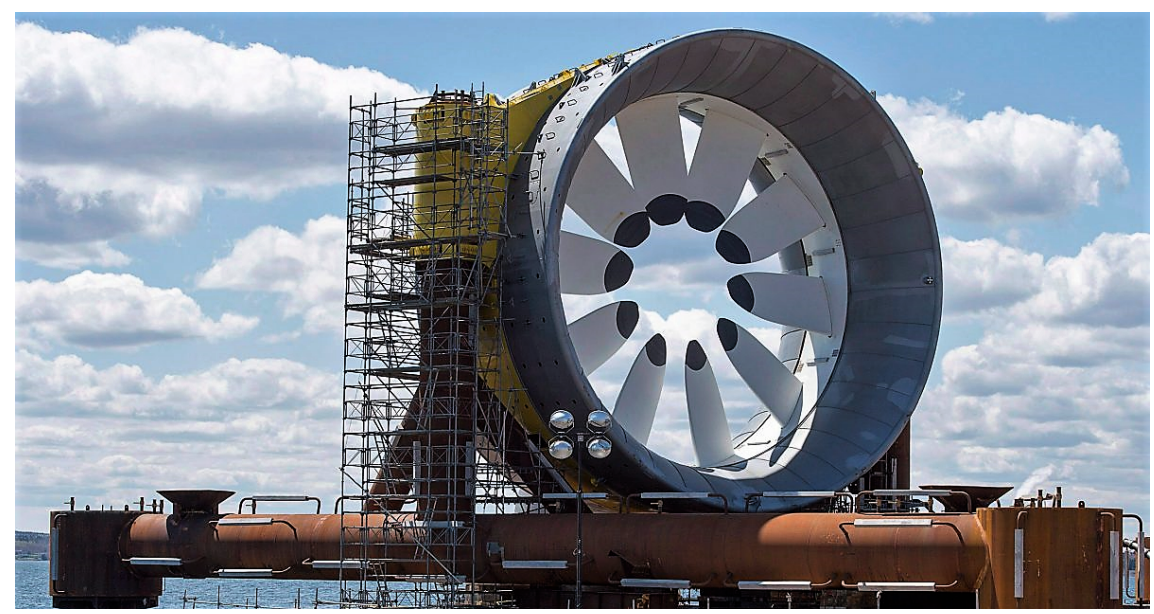

(a) 2 MW-rated turbine for the Cape Sharp project [11]

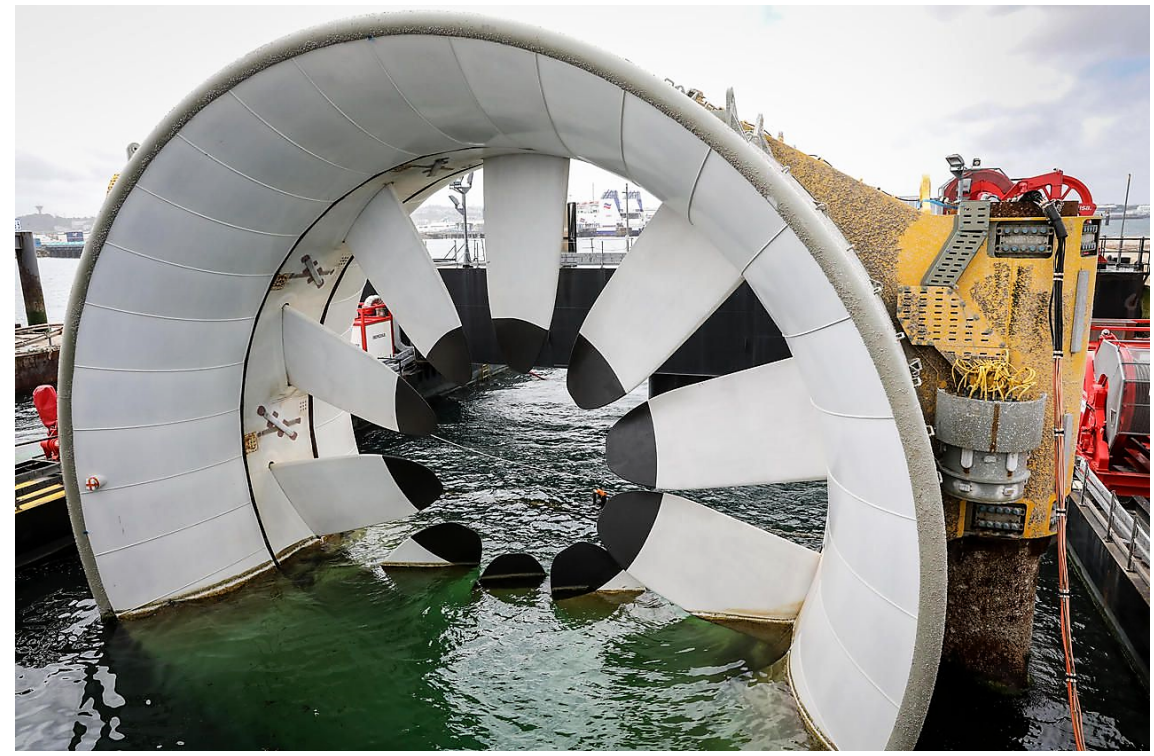

(b) $500 \mathrm{~kW}$-rated turbine at the Paimpol Bréhal site in Northern France [12]

Figure 1: The OpenHydro PS2 Ducted Turbine

\subsection{State of the Art}

Investigative analyses regarding the efficacy of ducted turbines have been mainly directed by numerical methodologies. Primarily instigated by wind turbine analyses, Phillips et al. [13, 14] developed a diffuser-type (or unidirectional) duct for the now-inactive Vortec Energy. Having undertaken both experimentation and CFD analyses for the turbine, good agreement was attained for velocity increases and pressure through the duct, yet power outcomes were found to be overestimated in modelling results, largely due to the implementation of the an actuator disc model together with the incapacity of the Standard $k-\varepsilon$ turbulence model in capturing certain separation effects. The effect of the disc loading was studied, which acknowledged that a large degree of intermittent flow separation occurred at 
the trailing third of the outer duct region in both experimentation and modelling. At high disc loading, where the outlet flow acts at a high velocity, however, it was noted that the separated region on the diffuser had been reduced.

Visser [15] analysed a ducted wind turbine design in test fields implementing a boundary layer control slot for pressure distribution within its diffuser section, whereas Hansen et al. [16] numerically established a CFD model of a diffuser by implementing an actuator disc in representation of a wind turbine. Werle and Presz [17] presented a theoretical basis, with numerical validation, of the potential improvements available from ducted flow configurations. Grassmann et al. [18, 19] analysed a diffuser consisting of two aerofoil sections utilising a similar segregated control slot by means of both experimentation and numerical simulation; Ohya et al. [20] tested a flanged diffuser to establish an optimal duct shape.

Shifting to their implementation in tidal turbine applications, Munch et al. [21] modelled a three-dimensional CFD simulation of a ducted turbine by means of a moving mesh methodology with blade actuality, albeit in a unidirectional aerofoil-shaped diffuser setting. In an effort of optimising both rotor and duct geometry, a nominal rotor power coefficient of 0.55 was attained for the four-bladed system. Gaden and Bibeau [22] investigated differing diffuser geometrical parameters by utilising an actuator disc representation region to extract axial momentum. Shives and Crawford [23] analysed a ducted CFD actuator disc model by varying the angle of attack of the unidirectional duct profile. In comparing two turbulence methodologies, RANS and LES, in low-wave conditions, Ahmed et al. [24] acknowledged the inaptness of resolving the wake turbulence by means of RANS turbulence modelling. Holst et al. [25] conducted a numerical study to analyse the wave-induced loads on tidal turbine blades at distinct angles of attack. This was then compared to BEM analyses, which depicted similar parameter outcomes.

In correspondence to the natural properties of tides, tidal turbine ducts have been industrially considered within a bidirectional setup. Setoguchi et al. [26] conducted an experimentational study utilising vacant ducts to investigate the most efficient shape for a two-way diffuser in a fluid-flow energy conversion system. Fleming and Willden [27] investigated the difference in velocity through the throat of a bidirectional duct in accordance to distinctive duct profiles, where the implemented turbine was represented as an actuator disc utilising CFD. In continuation, Allsop et al. [3, 4] developed a numerical blade-element momentum theory (BEMT) model to analyse flow through a duct in interaction with a high-solidity embedded rotor. Discretising the blade structure into elements, rather than portraying its entirety, the methodology analyses the turbine hydrodynamic performance by coupling the performance of the individual element sections, where the forces acting on the elements are then analysed utilising actuator disc/momentum theory coupled with the Bernoulli principle, to replicate the Venturi effect, with empirical corrections for duct interaction. Belloni et al. [5, 28, 29] investigated the effects of different bidirectional duct setups utilising a coupled BEM-CFD method to improve the capturing of mass and momentum continuity through the duct, avoiding the analytical limitations of the Bernoulli principle, together with representing turbulence effects.

The numerical analysis elaborated in this present study is a continuation of Borg et al. [30], which develops a real-scale CFD model to assess the hydrodynamic performance 
of a high-solidity open-centre tidal turbine within a bidirectional duct. This investigation evaluates the hydrodynamic effects of the duct on the turbine, where the individual turbine blades, rather than an area-averaged disc, are established, hence inferring the outcomes in quasi-real-ocean conditions. The hydrofoil geometry was provided by EDF R\&D to replicate the outcomes of a turbine similar to the design of the OpenHydro PS2 device. In addition, the 7-equation Wilcox Reynolds Stress 'Stress-Omega' Model (Stress- $\omega$ ) turbulence model was coupled to close the Navier-Stokes equation and analyse the anisotropic flow domain [31].

\section{Numerical Methodology}

In description of the procedure with which the hydrodynamic analysis was tackled, the turbine setup and flow parameters of the analysis are elaborated within the framework of the computational fluid dynamic solver numerical setup.

\subsection{Turbine Setup}

In an effort to attain a validated CFD model for tidal turbine applications, simulations were established to numerically replicate experimentation undertaken by Mycek et al. [32]. Identical blade, nacelle, and mast geometry were utilised within the model domain, illustrated in Figure 2, onto which a tetrahedral mesh was imprinted. The general dimensions of the turbine include a diameter $D$ of $0.7 \mathrm{~m}$, a nacelle length of $2.5 \mathrm{~m}$, and a mast length of $1 \mathrm{~m}$; supplementary descriptions may be attained within the literature. A distinction is present, however, between the physical aspects of the experimentation and the numerical model. In the prior, the nacelle mast protrudes through the free-surface to connect to the above support, whereas, in the latter, only the submerged length of the mast is considered, with a hemisphere at the tip to avoid tip-induced vortices. Hence, a physical assumption is present within which the drag forces on the mast are solely considered to be due to the current, rather than any additional wave \& wind factors. Albeit, due to the fact that waves were not induced in the experimentation, and that the setup was in a closed environment, the assumption that free-surface and topside effects were insignificant when considering the induced physics of the turbine was held. The parameters of the turbine and fluid flow were also instated from the literature, with a constant free-stream velocity of $0.8 \mathrm{~m} / \mathrm{s}$, a tipspeed ratio (TSR) range of $1.00-8.26$, an inlet turbulent length scale of $1 \mathrm{~m}$, and an inlet turbulence intensity of $3 \%$.

In continuation, upon validation, the model settings were implemented for the analysis of a ducted eight-bladed tidal turbine, similar to the design of the OpenHydro PS2 device. The general dimensions of the turbine were provided by EDF R\&D, which describe a rotor diameter of $12 \mathrm{~m}$, a duct diameter of $15 \mathrm{~m}$, and a duct length of $10 \mathrm{~m}$, as illustrated in Figure 3; supplementary descriptions are available in Table 1. The hydrofoil sections comprising the blades consisted of a flat-plate design with rounded edges. The geometry is quasi-identical to Allsop et al. [3], being slightly adapted to attain a more homogeneous blade surface, as described in Table 2. The turbine geometry was implemented within the model domain, onto which a tetrahedral mesh was imprinted. In resemblance to the validation procedure, the physical model, was designed to consist of identical domain layout 
and boundary conditions as the three-bladed HATT numerical model. The parameters of the turbine and fluid flow were instated from real-world data, recommended by EDF R\&D, as reported in Neill et al. [33] and Bahaj and Myers [34], with maximum acquired spring tide velocities of $4.0 \mathrm{~m} / \mathrm{s}$, and surface velocities of $5.0 \mathrm{~m} / \mathrm{s}$, respectively. For this reason, the free-stream velocity range initiated varied between $1 \mathrm{~m} / \mathrm{s}-7 \mathrm{~m} / \mathrm{s}$, in iterations of 1.5 $\mathrm{m} / \mathrm{s}$, with an inlet turbulence intensity of $3 \%$ and an inlet turbulent length scale of $1 \mathrm{~m}$, to acquire a definitive performance spectrum of the turbine. Within this free-stream velocity variation, a turbine TSR range of $1.00-2.50$ was considered as the nominal region, both due to high-solidity predictions specified by Betz [6], and analysis by Allsop et al. [4].

\subsection{Numerical Setup}

Inferred as fully submerged systems within a constant fluid flow, the models were founded under the premise that no far-stream effects are in interaction with the turbines, hence unaffected by the implications of the free-stream and the seabed; this was purposely implemented to acknowledge the performance of the turbines under ideal, consistent temporal conditions.

Implementing the use of the commercial solver ANSYS Fluent 16.1, the CFD physical models were designed to consist of a cuboidal external domain layout, imposed with relevant boundary conditions. The CFD solver was utilised in computing time-averaged representations of the continuity and Navier-Stokes equations, which govern the three-dimensional,

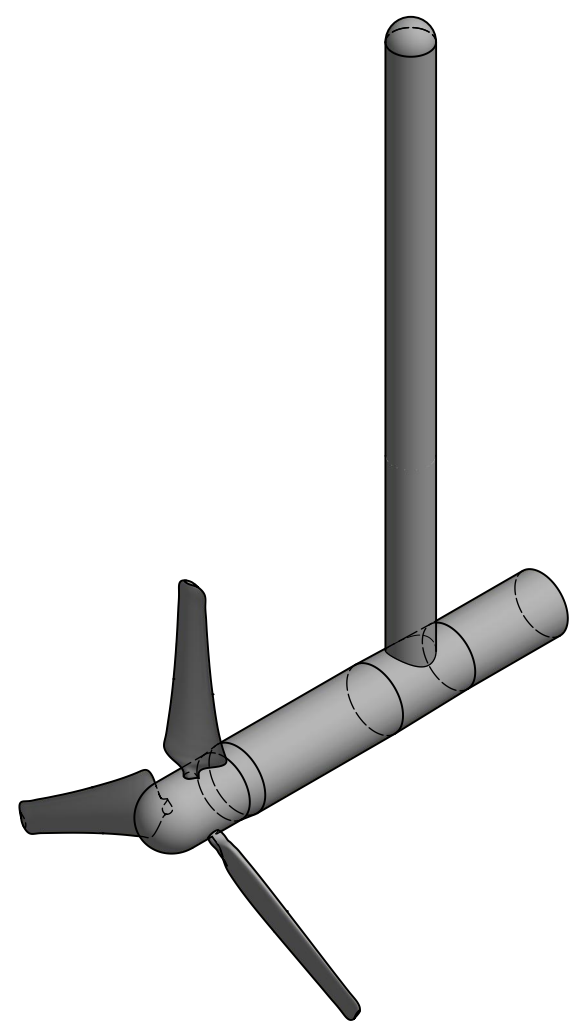

Figure 2: Rendered three-dimensional CAD representation of the horizontal-axis tidal turbine [32] utilised for the validation of the CFD model 


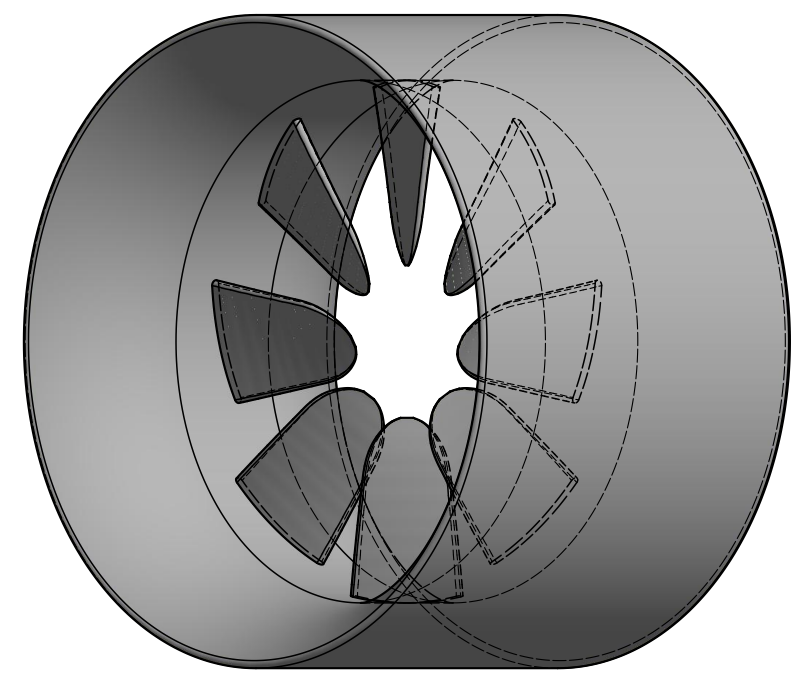

(a) Rendered three-dimensional CAD representation
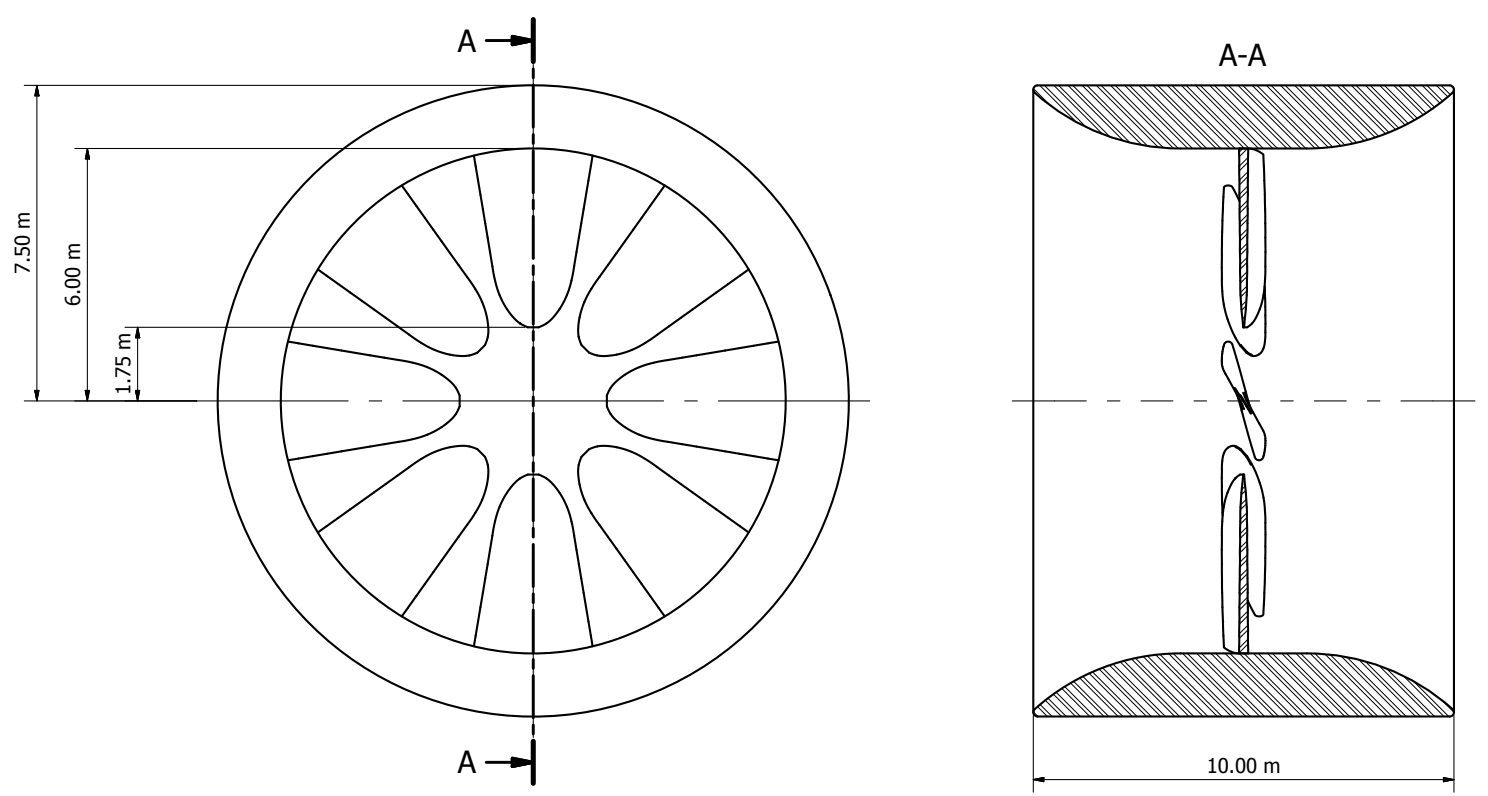

(b) First-angle sectioned projection

Figure 3: Geometrical model of the ducted high-solidity tidal turbine utilised for the CFD analyses

unsteady, incompressible fluid flow, along with a Reynolds-Averaged Navier-Stokes (RANS) turbulence model utilised in mathematical closure to represent flow property fluctuation. For both turbine models, the domain surrounding the turbine rotor was segregated from the global domain in an effort to induce a moving-mesh model with rotation at the turbine rotor relative to a stationary outer domain, with interfaces between the two domains. No-slip wall conditions were instigated on the rotor, mast and nacelle, and duct structures, whereas slip 


\begin{tabular}{ccc}
\hline Description & Validation Turbine [32] & Ducted Turbine \\
\hline \hline Blade Profile & NACA 63418 & Round-Edged Flat Plate \\
Rotor Radius $\left(R_{r t r}\right)$ & $0.35 \mathrm{~m}$ & $6.00 \mathrm{~m}$ \\
Hub Radius $\left(R_{h u b}\right)$ & $0.046 \mathrm{~m}$ & $1.75 \mathrm{~m}$ \\
Duct Radius $\left(R_{d c t}\right)$ & $\mathrm{N} / \mathrm{A}$ & $7.50 \mathrm{~m}$ \\
Hub Length $\left(L_{h u b}\right)$ & $0.72 \mathrm{~m}$ & $\mathrm{~N} / \mathrm{A}$ \\
Duct Length $\left(L_{d c t}\right)$ & $\mathrm{N} / \mathrm{A}$ & $10.00 \mathrm{~m}$ \\
Linear Velocity $\left(U_{\infty}\right)$ & $0.8 \mathrm{~m} / \mathrm{s}$ & $1-7 \mathrm{~m} / \mathrm{s}$ \\
Tip-Speed Ratio $(T S R)$ & $1.00-8.26$ & $1.00-2.50$ \\
Rotor Solidity $\left(S_{r t r}\right)$ & 0.132 & 0.614 \\
Rotational Orientation & counter-clockwise & $\mathrm{clockwise}$ \\
Reynolds Number $\left(R e_{\infty}\right)$ & $0.279 \cdot 10^{6}$ & {$[5.97-41.80] \cdot 10^{6}$} \\
Reynolds Number $\left(R e_{L}\right)$ & $0.573 \cdot 10^{6}$ & {$[9.95-69.67] \cdot 10^{6}$} \\
Reynolds Number $\left(R e_{c h}\right)$ & {$[0.049-0.245] \cdot 10^{6}$} & {$[3.15-37.49] \cdot 10^{6}$} \\
\hline
\end{tabular}

Table 1: Summary of Turbine Model Descriptions

conditions were instigated on the boundaries of the domain.

In a similar layout employed by Mason-Jones et al. [14], the dimensions of the computational domain were contrived to attain a numerical blockage ratio $\left(\alpha_{b l}\right)$ of less than $2 \%$ to the turbine to ascertain the absence of far-field effects, replicating real-ocean conditions. This corresponded to a cross-sectional domain dimension of $7 D$ along the edges of the quadratic face, with the rotor cylindrical domain positioned in the centre of the vertical plane. In consideration of the wake analysis, a domain length of $9 D$ was instituted, where the inlet and outlet planes were situated at a distance $3 D$ upstream and $6 D$ downstream of the turbine, respectively, as illustrated in Figure 4a. The time-step considered was designated in relation to the tip-speed ratio of the rotor, where each transient iteration is temporally equivalent to one-half of a degree of a turbine revolution, therefore attained by:

$$
\Delta t_{\text {step }}=\frac{1}{2} \times \frac{2 \pi}{360 \times \Omega_{\text {sys }}}
$$

where $\Delta t_{\text {step }}$ is the time-step and $\Omega_{\text {sys }}$ is the rotational velocity of the system. Having undertaken a time-step independence analysis, this was found to suffice both in sustaining stability in the model, and in achieving a sufficient Courant number at regions of interest throughout the domain.

Turbulence was modelled by means of the Reynolds-Stress Model (RSM) 'Stress-Omega' turbulence model. This model was opted for three distinctive reasons: i) as a variable, the specific dissipation rate $(\omega)$ is superior to the dissipation rate $(\varepsilon)$ in establishing the turbulent length scale as a result of its capacity in modelling all degrees of production, redistribution, and dissipation of turbulent kinetic energy within the flow, ii) mathematically, $\omega$ does not require the implementation of near-wall treatment equations to model the boundary layer at non-slip surfaces, as the function is able to be integrated to the wall, displaying greater 


\begin{tabular}{cccc}
\hline$r_{r t r} / R_{r t r}$ & $c_{r t r} / R_{r t r}$ & Pitch $\left(^{\circ}\right)$ & $t_{r t r} / c_{r t r}(\%)$ \\
\hline \hline 0.2917 & 0.0500 & 32.00 & 6.67 \\
0.3067 & 0.1208 & 31.66 & 4.14 \\
0.3350 & 0.1908 & 30.98 & 5.24 \\
0.3633 & 0.2383 & 30.30 & 6.29 \\
0.3917 & 0.2750 & 29.62 & 6.67 \\
0.4200 & 0.3025 & 28.94 & 7.16 \\
0.4483 & 0.3233 & 28.26 & 7.22 \\
0.4767 & 0.3383 & 27.58 & 6.90 \\
0.5050 & 0.3500 & 26.90 & 7.14 \\
0.5333 & 0.3583 & 26.22 & 6.98 \\
0.5617 & 0.3667 & 25.54 & 6.82 \\
0.5900 & 0.3750 & 24.86 & 7.11 \\
0.6183 & 0.3833 & 24.18 & 6.96 \\
0.6467 & 0.3917 & 23.50 & 6.81 \\
0.6750 & 0.4000 & 22.82 & 7.08 \\
0.7033 & 0.4083 & 22.14 & 6.94 \\
0.7317 & 0.4167 & 21.46 & 7.20 \\
0.7600 & 0.4250 & 20.78 & 7.06 \\
0.7883 & 0.4333 & 20.10 & 6.92 \\
0.8167 & 0.4417 & 19.42 & 7.17 \\
0.8450 & 0.4500 & 18.74 & 7.04 \\
0.8733 & 0.4583 & 18.06 & 6.91 \\
0.9017 & 0.4667 & 17.38 & 7.14 \\
0.9300 & 0.4750 & 16.70 & 7.02 \\
0.9583 & 0.4833 & 16.02 & 6.90 \\
0.9867 & 0.4917 & 15.34 & 7.12 \\
1.0000 & 0.5000 & 15.00 & 7.00 \\
& & &
\end{tabular}

Table 2: Detailed ducted turbine blade profile descriptions

accuracy within the log-linear range of the law-of-the-wall y-plus $\left(y^{+}\right)$spectrum, and iii) as a result of its non-implementation of the Boussinesq approximation, the model analyses all Reynolds stresses in three-dimensional space, prompting superiority in analysing anisotropic flows, such as flows over curved surfaces, flows in rotating fluids, and flows in ducts with secondary (rotational) motion [31]. Albeit the advantages of the Stress- $\omega$ turbulence model, specifically in wall-bounded flows, a significant drawback it presents is the potential oversensitivity in shear flow at high Reynolds numbers, similar to the Standard $k-\omega$ turbulence model, hence potentially portraying unphysically higher outcomes under exaggerated physics within the fluid-structure interaction phenomena.

In accompaniment to the Stress- $\omega$ model, the SIMPLE pressure-velocity coupling scheme setting, the Green-Gauss Node Based gradient, Pressure Staggering Option pressure, Sec- 


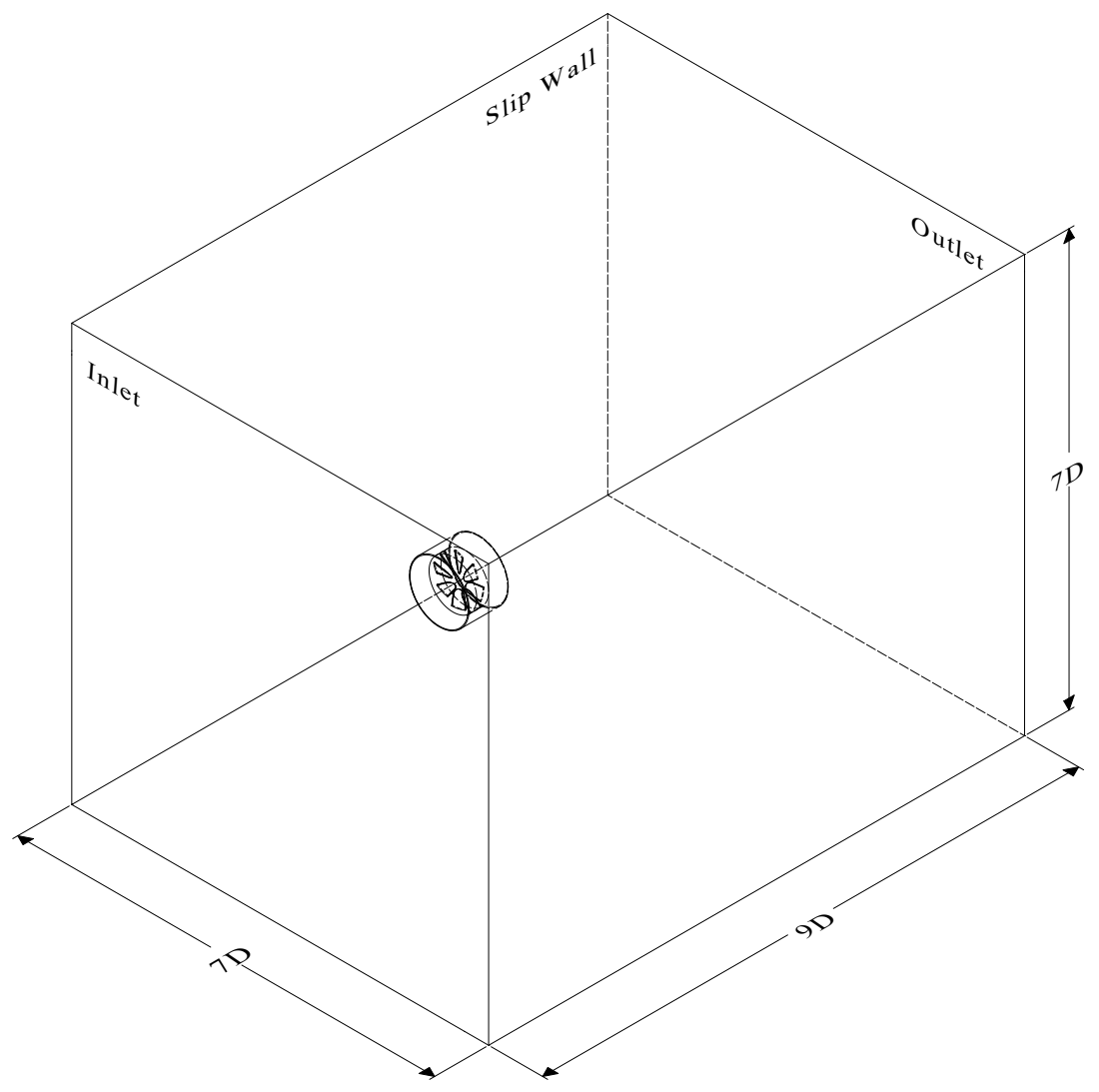

(a) Layout of the ducted turbine domain

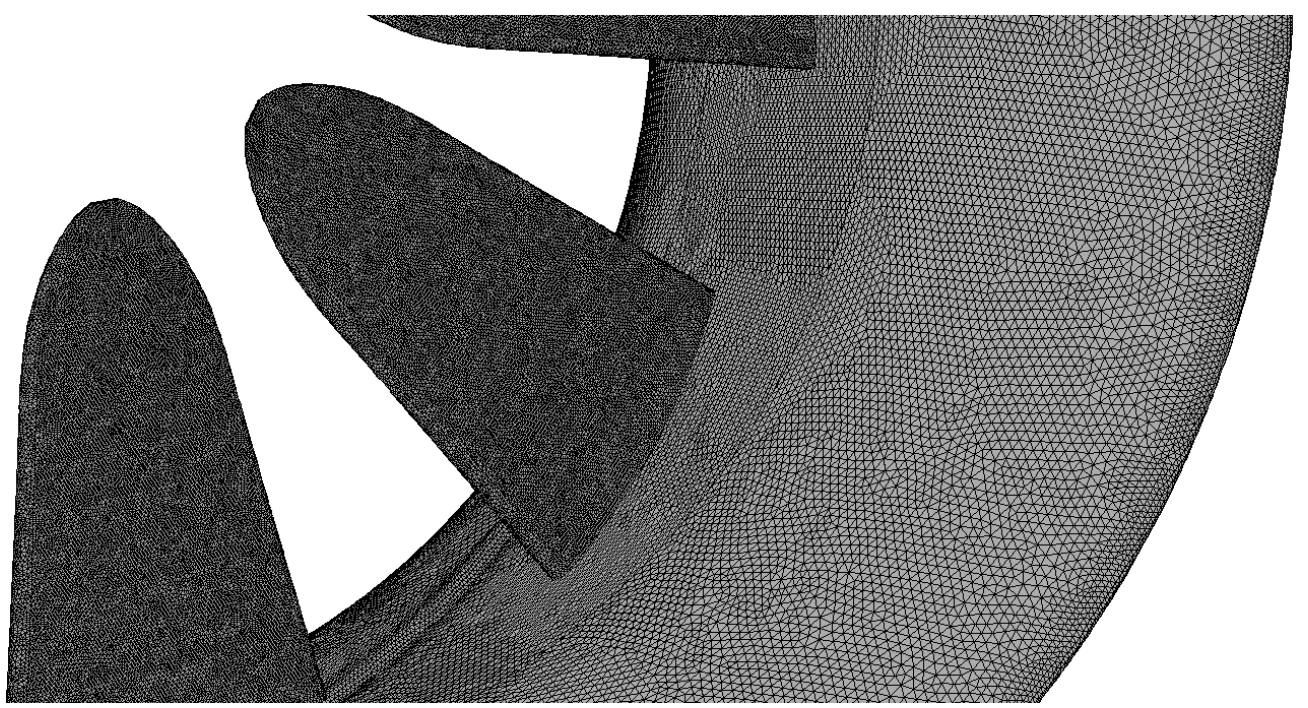

(b) Geometric tessellation of the mesh on the duct and rotor blades

Figure 4: Geometrical model of the ducted high-solidity tidal turbine utilised for the CFD analyses 
ond Order Upwind momentum, Second Order Upwind specific dissipation rate, and Second Order Upwind Reynolds stresses spatial discretisation settings, and the Bounded Second Order Implicit transient formulation setting were utilised as the solution methodologies. It should be stated that the dissipation rate was altered to Second Order from First Order settings once the resultant parameter outputs were steady in time, as initially running with the more accurate setting would result in the simulation to fail within the first few time-steps. Furthermore, the pressure setting was also altered from Second Order to Pressure Staggering Option once the resultant parameter outputs were steady in time; the prior setting greatly underestimates the parameters. In addition, for the successful use of the Stress- $\omega$ turbulence model with these settings, a quality mesh is required. Through empirical means, it was acknowledged that a mesh with skewness higher than 0.79 resulted in the failure of the simulation, highlighting the limited robustness of the turbulence model; other mesh qualimetrics had no such an effect.

A mesh independence procedure was primarily carried out on the three-bladed HATT by considering the parameter with the highest degree of dynamics; the highest TSR condition. Subsequent to the procedure, the final average surface mesh count utilised was marginally above 87,000 cell faces per blade, with dense volumetric cells around the turbine and within the wake, to achieve a total mesh count of 13.5 million for the three-bladed HATT, over 8 million of which within the turbine rotating region. An identical procedure was undertaken for the ducted turbine, attaining an average surface mesh of slightly above 94,000 cell faces per blade, illustrated in Figure 4b, with more than 18.5 million volumetric cells within the entire domain, over 10 million of which within the turbine rotating region. The mesh was implemented with a prism layer at non-slip surfaces with an appropriate cell height to achieve a y-plus value of $60 \leqslant y^{+} \leqslant 500$ across the blades and duct. This range was considered due to the high Reynolds number $\left(>10^{6}\right)$ of the system, hence modelling the viscous sublayer was abdicated to reduce computation time.

The CFD computations were performed using the ARCHIE-WeSt cluster facility at the University of Strathclyde by running two Intel Xeon Gold $61382.00 \mathrm{GHz}$ computational nodes, with 40 cores and $192 \mathrm{~GB}$ of RAM per node per simulation. A ducted turbine simulation was completed within roughly 75 wall-clock hours, equivalent to 3,000 core-hours, hence resulting in an average of 5 wall-clock hours per turbine rotation.

\section{Numerical Model Characterisation}

The characterisation of the numerical model, where the formulae utilised in relation to the physical aspects of the tidal turbine, and in analysis of the fluid continuum, are specified.

\subsection{Physical Modelling}

In consideration of the analysis of a physical turbine, notable definitions concerning the resultant performance outcomes, in terms of the boundary conditions employed, are identified. Utilised to attain quasi-real-ocean conditions, the blockage ratio $\left(\alpha_{b l}\right)$ is defined as a correlation between the device reference area $\left(A_{d v c}\right)$ and the domain sectional area 
$\left(A_{d m n}\right):$

$$
\alpha_{b l}=\frac{A_{d v c}}{A_{d m n}}=\frac{\pi R_{d v c}^{2}}{L_{d m n}^{2}}
$$

where $R_{d v c}$ is the device radius, and $L_{d m n}$ is the length of the quadratic cross-sectional area of the computational domain.

The tip-speed ratio $(T S R)$ is established as an equivalence between the linear blade-tip velocity and the free-stream velocity:

$$
T S R=\frac{\left|\Omega_{s y s}\right| R_{r t r}}{U_{\infty}}=\frac{\left|\Omega_{x}\right| R_{r t r}}{U_{\infty}}
$$

where $\Omega_{\text {sys }}$ is the system rotational speed, hence $\Omega_{x}$ being the axial angular velocity, and $R_{r t r}$ is the rotor radius. A distinction must be made between the device radius and the rotor radius when modelling ducted turbines. In the case of a ducted turbine, $R_{d v c} \neq R_{r t r}$, but $R_{d v c}=R_{d c t}$, contrary to a non-ducted turbine, where $R_{d v c}=R_{r t r}$.

The rotor solidity $\left(S_{r t r}\right)$ of a turbine is applied to establish a ratio between the projected area of the static rotor in the axial direction $\left(A_{b l d-z}\right)$ and the total area of the rotor rotation $\left(A_{r t r}\right)$ :

$$
S_{r t r}=\frac{A_{b l d-z}}{A_{r t r}}=\frac{A_{b l d-z}}{\pi R_{r t r}^{2}}
$$

The radius-based Reynolds number $\left(R e_{\infty}\right)$ is utilised to acquire a dimensionless representation of the fluid-structure interaction by considering the fluid inertial and viscous forces as a bluff body:

$$
R e_{\infty}=\frac{\rho U_{\infty} R_{d v c}}{\mu}
$$

where $\rho$ is the fluid density, $U_{\infty}$ is the free-stream velocity, and $\mu$ is the fluid dynamic viscosity.

The length-based Reynolds number $\left(R e_{L}\right)$ is utilised to acquire a dimensionless representation of the fluid-structure interaction by considering the fluid inertial and viscous forces along the length of the structure:

$$
R e_{L}=\frac{\rho U_{\infty} L_{d v c}}{\mu}
$$

where $L_{d v c}$ is the device length, and hence is equal either to the hub length $\left(L_{h u b}\right)$ for the HATT, or the duct length $\left(L_{d c t}\right)$ for the ducted turbine.

In continuation, the chord-based Reynolds number $\left(R e_{c h}\right)$ is utilised to acquire a dimensionless representation of the fluid-structure interaction in terms of the resultant velocity at $75 \%$ of the radius of the rotating device blades:

$$
R e_{c h}=\frac{\rho c_{75 \%} \sqrt{\left(U_{\infty}\right)^{2}+\left(\Omega_{x} R_{75 \%}\right)^{2}}}{\mu}
$$

where $c_{75 \%}$ is the chord length at $75 \%$ of the blade radius $\left(R_{75 \%}\right)$. 
To determine the turbine capacity in converting the fluid free-stream energy into rotational energy, the power coefficient $\left(C_{P}\right)$ is established. This considers the mechanical rotational power attained by the device $\left(P_{d v c}\right)$ as a ratio of the maximum rotational power potentially acquired in the device area $\left(P_{\infty}\right)$ :

$$
C_{P}=\frac{P_{d v c}}{P_{\infty}}=\frac{M_{x} \Omega_{x}}{\frac{1}{2} \rho A_{d v c} U_{\infty}^{3}}=\frac{M_{x} \Omega_{x}}{\frac{1}{2} \rho \pi R_{d v c}^{2} U_{\infty}^{3}}
$$

where $M_{x}$ is the axial moment, also referred to as the rotor torque.

In relation to the power generated, the torque coefficient $\left(C_{Q}\right)$ evaluates the mechanical torque attained by the device $\left(Q_{d v c}\right)$ as a ratio of the maximum torque potentially acquired in the device area $\left(Q_{\infty}\right)$ :

$$
C_{Q}=\frac{Q_{d v c}}{Q_{\infty}}=\frac{M_{x}}{\frac{1}{2} \rho A_{d v c} R_{r t r} U_{\infty}^{2}}=\frac{M_{x}}{\frac{1}{2} \rho \pi R_{d v c}^{2} R_{r t r} U_{\infty}^{2}}
$$

In relation to the power generated, the mass flow-rate coefficient $\left(C_{\dot{m}}\right)$ evaluates the mass flow-rate through the rotor $\left(\dot{m}_{r t r}\right)$ as a ratio of the maximum mass flow-rate which may be potentially attained within the duct:

$$
C_{\dot{m}}=\frac{\dot{m}_{r t r}}{\rho A_{r t r} U_{\infty}}=\frac{\dot{m}_{r t r}}{\rho \pi R_{r t r}^{2} U_{\infty}}
$$

In continuation, the resultant thrust on the device induced in a direction parallel to the turbine axis contributes to the fluid-structure phenomenon. The thrust coefficient $\left(C_{T}\right)$ quantifies this as a function of the device thrust $\left(T_{d v c}\right)$ and the maximum thrust potentially induced upon the device area $\left(T_{\infty}\right)$ :

$$
C_{T}=\frac{T_{d v c}}{T_{\infty}}=\frac{F_{x}}{\frac{1}{2} \rho A_{d v c} U_{\infty}^{2}}=\frac{F_{x}}{\frac{1}{2} \rho \pi R_{d v c}^{2} U_{\infty}^{2}}
$$

where $F_{x}$ is the stream-wise axial force on the device.

In analysing the transient sinusoidal fluctuations in the performance outcomes, Strouhal's law was utilised in evaluating its relationship with the Reynolds number of the domain:

$$
S t=\frac{f_{v} L_{S t}}{U_{\infty}}
$$

where $S t$ is the Strouhal number, $f_{v}$ is the vortex-shedding frequency, and $L_{S t}$ is the Strouhal characteristic length.

\subsection{CFD Modelling}

Various degrees of flow complexities are induced as a result of the high Reynolds number ranges that large-scale tidal turbines physically approach. These ranges of turbulence are wholly elaborated by random fluctuations of the flow properties. Mean derivatives of the continuity and Navier-Stokes equations are utilised to model these flow properties, expressed as a vectorial summation of their mean and fluctuating terms, which, in turn, are equated by turbulence models to achieve mathematical closure. 


\subsubsection{Conservation Modelling}

Within three-dimensional Cartesian space, the conservation of mass is represented, in indicial notation, as:

$$
\frac{\partial \bar{\rho}}{\partial t}+\frac{\partial}{\partial x_{i}}\left(\bar{\rho} \tilde{u}_{i}\right)+\frac{\partial}{\partial x_{j}}\left(\bar{\rho} \tilde{u}_{j}\right)+\frac{\partial}{\partial x_{k}}\left(\bar{\rho} \tilde{u}_{k}\right)=0
$$

where $\bar{\rho}$ is the Reynolds-averaged density, $x_{i}$ is the Cartesian coordinate, $t$ is the elapsed time, and $\tilde{u}_{i}$ is the mass-averaged velocity $[35,36]$.

Assuming the fluid is incompressible, single-phase, and isotropic, due to the physicality of the hydrodynamic domain, within a Reynolds-averaged, tensor notation, the mass continuity equation takes the form of:

$$
\frac{\partial U_{i}}{\partial x_{i}}=0
$$

where $U_{i}$ is the Reynolds-averaged velocity.

In continuation, the conservation of momentum within three-dimensional, tensor notation is represented as:

$$
\frac{\partial\left(\bar{\rho} \tilde{u}_{i}\right)}{\partial t}+\frac{\partial}{\partial x_{j}}\left(\bar{\rho} \tilde{u}_{i} \tilde{u}_{j}-\bar{\sigma}_{i j}\right)=0
$$

where $\bar{\sigma}_{i j}$ is the total stress tensor. The above equation, in Reynolds-averaged format, then becomes:

$$
\rho \frac{\partial U_{i}}{\partial t}+\rho \frac{\partial}{\partial x_{j}}\left(U_{i} U_{j}+\overline{u_{j}^{\prime} u_{i}^{\prime}}\right)-\frac{\partial}{\partial x_{j}}\left(\sigma_{i j}\right)=0
$$

where $U_{i}$ is the Reynolds-averaged velocity, $u_{i}^{\prime}$ is the fluctuating velocity component, and $\sigma_{i j}$ is the Reynolds-averaged total stress tensor, which, for incompressible flows, under the absence of volume dilation (or elastic stress), is specified as the tensor summation of the static pressure and viscous stress tensor:

$$
\sigma_{i j}=-\delta_{i j} P_{s}+\mu\left(\frac{\partial U_{i}}{\partial x_{j}}+\frac{\partial U_{j}}{\partial x_{i}}\right)
$$

where $\delta_{i j}$ is the Kronecker delta (or unit tensor), $P_{s}$ is the static pressure, and $\mu$ is the dynamic viscosity. The momentum equation, therefore, may be presented as:

$$
\rho \frac{\partial U_{i}}{\partial t}+\rho U_{j} \frac{\partial U_{i}}{\partial x_{j}}=-\frac{\partial P_{s}}{\partial x_{i}}+\frac{\partial}{\partial x_{j}}\left(\mu\left(\frac{\partial U_{i}}{\partial x_{j}}+\frac{\partial U_{j}}{\partial x_{i}}\right)-\rho \overline{u_{j}^{\prime} u_{i}^{\prime}}\right)
$$

where $-\rho \overline{u_{j}^{\prime} u_{i}^{\prime}}=\rho \tau_{i j}$, and is known as the Reynolds-stress tensor, with $\tau_{i j}=-\overline{u_{j}^{\prime} u_{i}^{\prime}}$ being the specific Reynolds-stress tensor. 


\subsubsection{Turbulence Modelling}

In introducing mathematical capacities to model the specific Reynolds-stress tensor, and hence, model turbulence within the computational domain, measures are implemented to regard parametric effects of flow history transience, together with the development of convection, production, and body-force terms, in response to physical disparities in strain rate, and the resultant diffusion, redistribution, and dissipation [31].

In an effort to introduce the aspects of turbulence, the Wilcox Stress- $\omega$ turbulence model was utilised in this CFD model, in which the specific Reynolds-stress tensor $\left(\tau_{i j}\right)$, in tensor notation, is established by:

$$
\begin{aligned}
\rho \frac{\partial \tau_{i j}}{\partial t}+\rho U_{k} \frac{\partial \tau_{i j}}{\partial x_{k}}= & \frac{\partial}{\partial x_{k}}\left[\left(\mu+\frac{\mu_{T}}{\sigma_{k}}\right) \frac{\partial \tau_{i j}}{\partial x_{k}}\right]-\rho P_{i j}-\rho \Pi_{i j}+ \\
& \frac{2}{3} \beta^{*} \rho \omega k \delta_{i j}-2 \rho \omega_{k}\left(\tau_{j m} \epsilon_{i k m}+\tau_{i m} \epsilon_{j k m}\right)
\end{aligned}
$$

where $\rho$ is the Reynolds-averaged density, $P_{i j}$ is the Reynolds stress production tensor, $t$ is the elapsed time, $x_{i}$ is the Cartesian coordinate, $k$ is the kinetic energy of turbulent fluctuations per unit mass (or turbulence kinetic energy), $\omega$ is the specific dissipation rate, $\Pi_{i j}$ is the pressure-strain correlation tensor, $\mu$ is the dynamic viscosity, and $\mu_{T}$ is the eddy viscosity, $\omega_{k}$ is the rotation vector, and $\epsilon_{i j k}$ is the Levi-Civita pseudotensor; $\beta^{*}$ and $\sigma_{k}$ are closure coefficients.

The specific dissipation rate $(\omega)$ is defined by:

$$
\rho \frac{\partial \omega}{\partial t}+\rho U_{j} \frac{\partial \omega}{\partial x_{j}}=\frac{\partial}{\partial x_{j}}\left[\left(\mu+\frac{\mu_{T}}{\sigma_{k}}\right) \frac{\partial \omega}{\partial x_{j}}\right]+\alpha \frac{\rho \omega}{k} \tau_{i j} \frac{\partial U_{i}}{\partial x_{j}}-\beta_{o} f_{\beta} \rho \omega^{2}
$$

where $f_{\beta}$ is utilised to implement the mean rotation tensor $\left(\Omega_{i j}\right) ; \alpha$ and $\beta_{o}$ are closure coefficients.

The pressure-strain correlation tensor $\left(\Pi_{i j}\right)$ is defined by:

$$
\Pi_{i j}=\beta^{*} C_{1} \omega\left(\tau_{i j}+\frac{2}{3} k \delta_{i j}\right)-\hat{\alpha}\left(P_{i j}-\frac{1}{3} P_{k k} \delta_{i j}\right)-\hat{\beta}\left(D_{i j}-\frac{1}{3} P_{k k} \delta_{i j}\right)-\hat{\gamma} k\left(S_{i j}-\frac{1}{3} S_{k k} \delta_{i j}\right)
$$

where $S_{i j}$ is the mean strain-rate tensor and $D_{i j}$ is the rotation production tensor; $C_{1}, \hat{\alpha}, \hat{\beta}$, and $\hat{\gamma}$ are closure coefficients.

Specificities with regards to tensor definitions, and closure coefficients and derivatives may be further established in the referenced literature [31, 35].

\section{Performance Validation of a Horizontal-Axis Tidal Turbine}

In validation of the CFD model, the numerical outcomes of a three-bladed HATT within a TSR range of $1.00-8.26$ in open-water conditions, in relevance to the power coefficient, torque coefficient, thrust coefficient, and wake velocity profiles from near- to far-wake, were ascertained in comparison to Mycek et al. [32] utilising the coefficient of determination $\left(R^{2}\right)$ statistical methodology. 


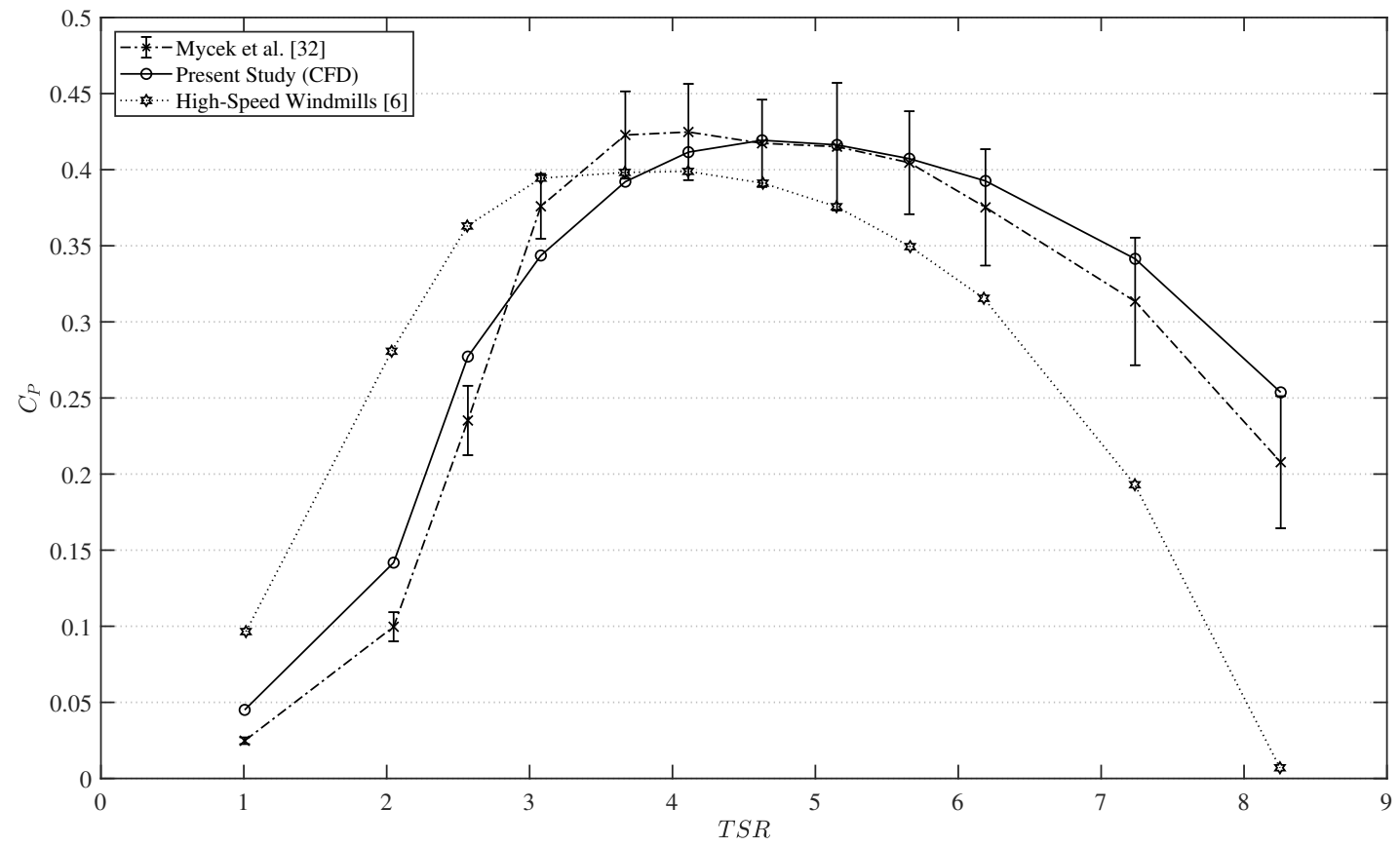

(a) Power coefficient $\left(C_{P}\right)$ in relation to TSR

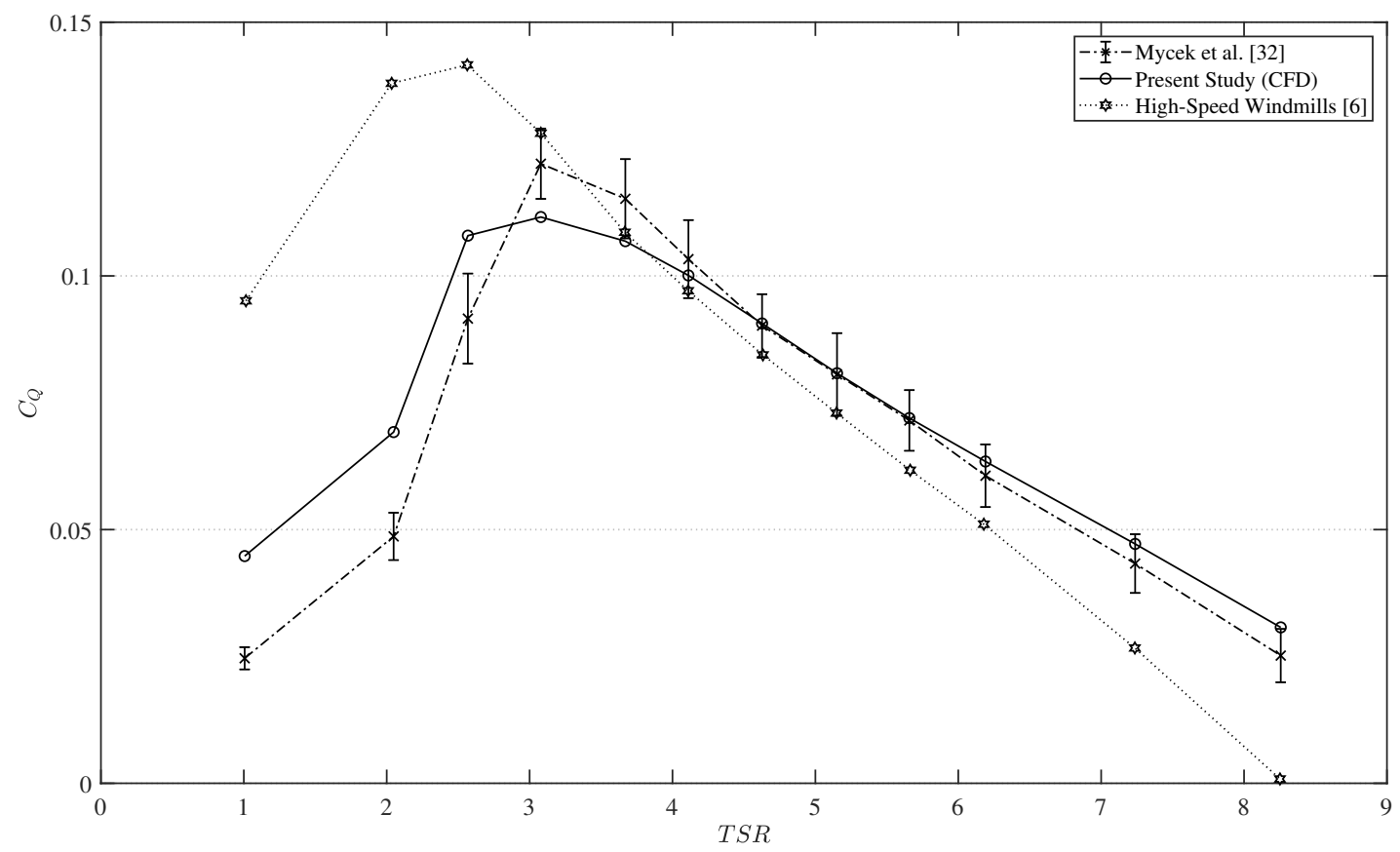

(b) Torque coefficient $\left(C_{Q}\right)$ in relation to TSR

Figure 5: Comparative evaluation of the current horizontal-axis tidal turbine CFD model with experimentation [32], and high-speed windmills [6] 


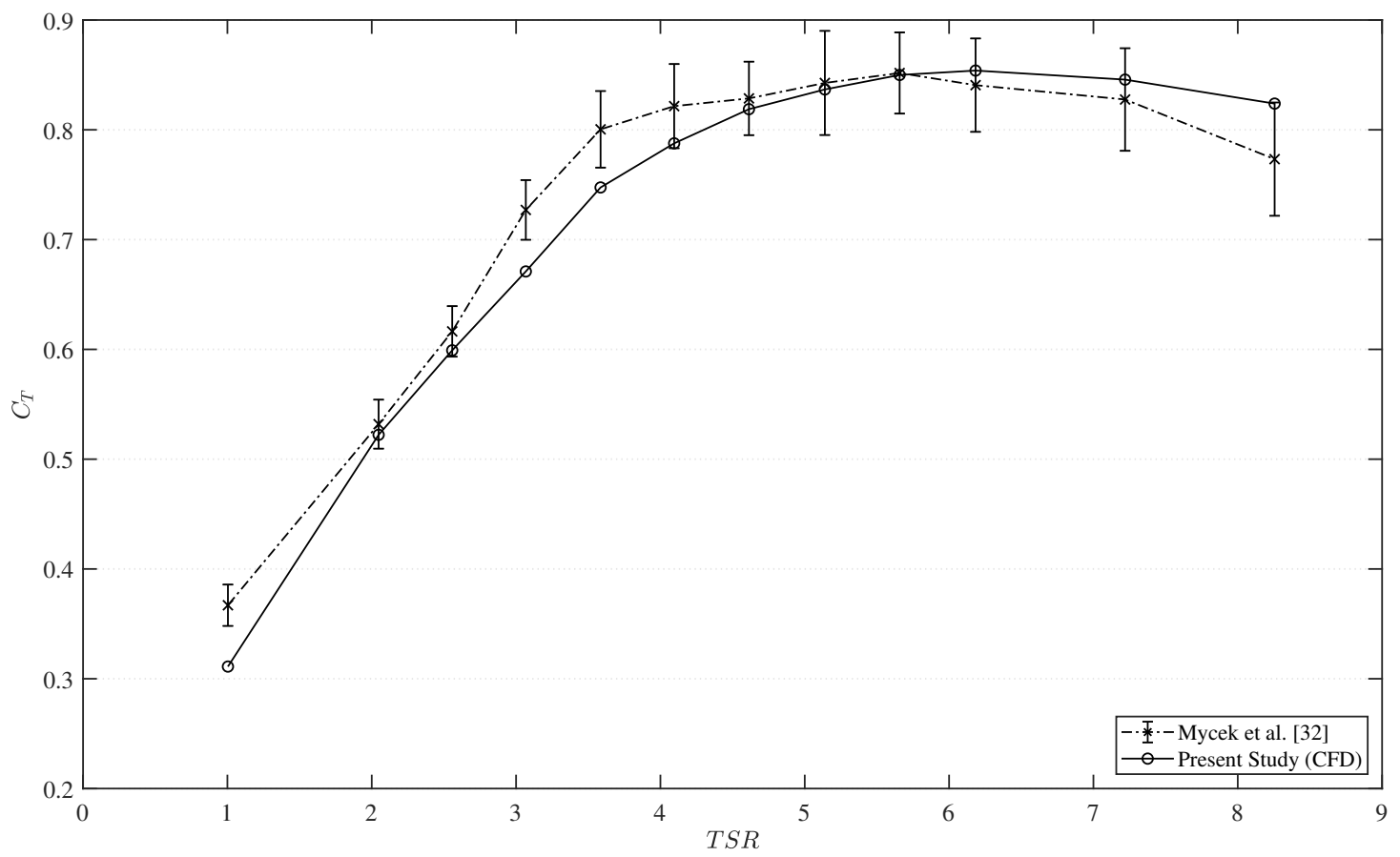

Figure 6: Comparative evaluation of the current horizontal-axis tidal turbine CFD model with experimentation [32] for thrust coefficient $\left(C_{T}\right)$ in relation to TSR

\subsection{Power Coefficient}

Primarily, the power coefficient $\left(C_{P}\right)$ curve established by numerical means, illustrated in Figure 5a, attained an $R^{2}$ of 0.955 as a degree of similarity with experimentation pertaining to the data points within the entire curve, and a 0.965 degree of similarity within the nominal TSR range (TSR 3.08 - 5.66). As the limits of the error bars in the graphs represent one standard deviation $(\sigma)$, all CFD data points analysed fell within $2 \sigma_{C_{P}}$ of the experimentation points, hence within the $95^{t h}$-percentile confidence range, except for low TSR values of 1.00 and 2.05. Furthermore, the torque coefficient $\left(C_{Q}\right)$ curve established by numerical means, illustrated in Figure 5b, attained an $R^{2}$ of 0.889 as a degree of similarity with experimentation pertaining to the data points within the entire curve, and a 0.965 degree of similarity within the nominal TSR range (TSR 3.08 - 5.66). All CFD data points analysed fell within $2 \sigma_{C_{Q}}$ of the experimentation points, hence within the $95^{t h}$-percentile confidence range, except for low TSR values of 1.00 and 2.05. Specifying the affiliated physics between the validation systems and large-scale systems, the numerical and experimentation data sets are compared to high-speed windmills presented in the Betz [6] technical memorandum, indicating good comparison specifically within the trends of the performance curves.

\subsection{Thrust Coefficient}

In continuation, the thrust coefficient $\left(C_{T}\right)$ curve established by numerical means, in consideration of the rotor, nacelle, and mast, illustrated in Figure 6 , attained an $R^{2}$ of 


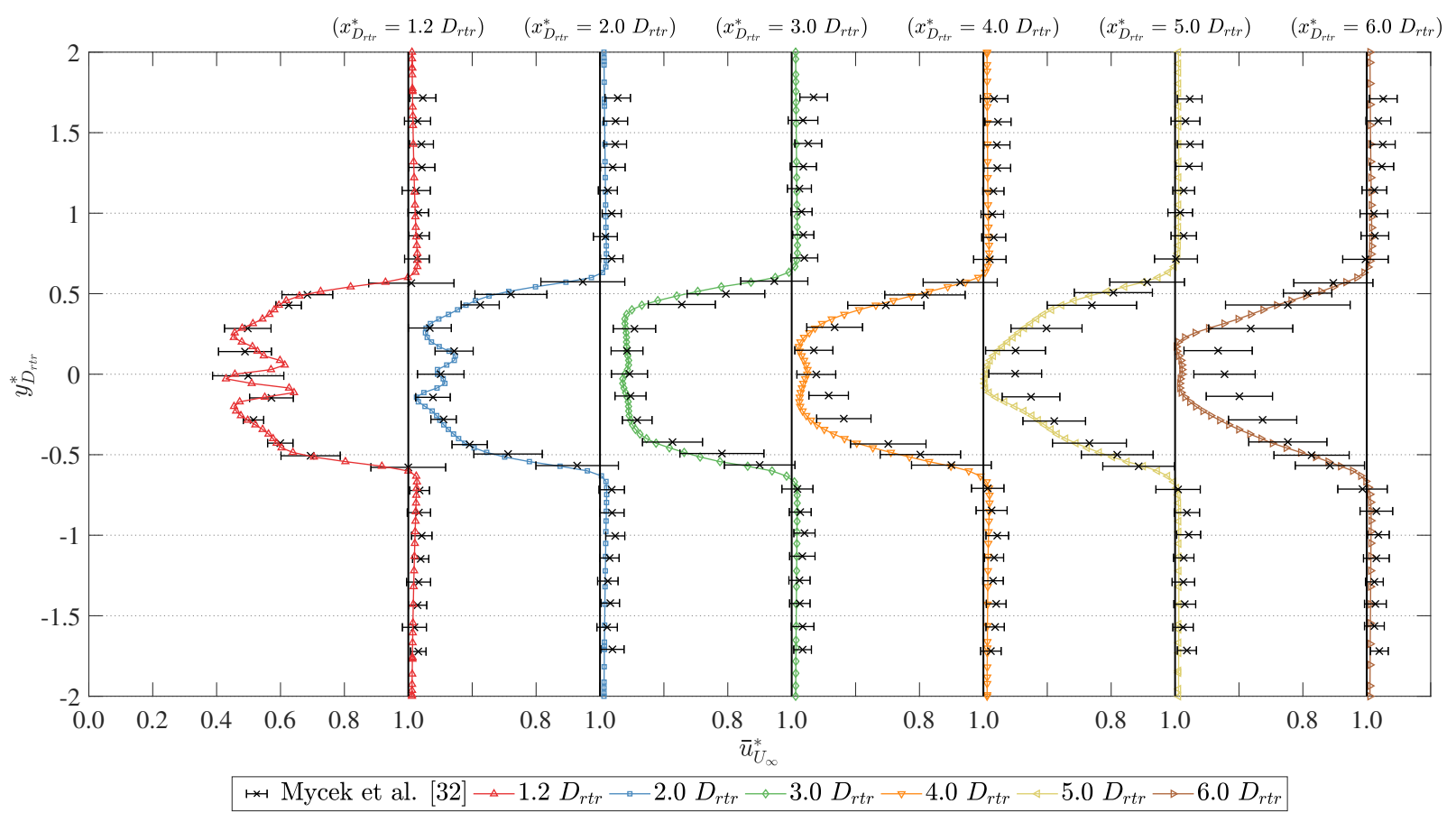

Figure 7: Comparative evaluation of the current horizontal-axis tidal turbine CFD model with experimentation [32] for the wake velocity profiles at distinct downstream displacements $\left(x_{D_{r t r}}^{*}\right)$ at TSR 3.67

0.946 as a degree of similarity with experimentation pertaining to the data points within the entire curve, and a 0.841 degree of similarity within the nominal TSR range (TSR 3.08 - 5.66). All CFD data points analysed fell within $2 \sigma_{C_{T}}$ of the experimentation points, hence within the $95^{\text {th }}$-percentile confidence range, except for the low TSR value of 1.00. The comparatively substantial discrepancy between the numerical and experimentation thrust coefficient may have come about due to the inaccuracy of the mast geometry implemented within the model. No data regarding the position and dimensions of the mast were noted in the literature, and hence, creating the complete geometry was executed with calculated estimations; in addition, fairings were utilised during the experimentation, whereas a bare mast was created in the numerical model, hence skewing the resultant data comparison.

\subsection{Wake Velocity Profiles}

Further to the performance evaluation, the wake velocity profiles established by numerical means, illustrated in Figure 7 , attained an $R^{2}$ of 0.982 and 0.863 as a degree of similarity with experimentation pertaining to the data points within the entire curve, at near-wake and farwake respectively. All the CFD data points analysed fell within $2 \sigma_{C_{T}}$ of the experimentation points, hence within the $95^{\text {th }}$-percentile confidence range. The comparatively substantial discrepancy between the numerical and experimentation at the far-wake may be a result of the underestimation of the dissipation rate within the fluid, due to the implementation of the turbulence model, hence overestimating the resultant kinetic energy within the wake. 


\section{Performance of the Ducted High-Solidity Tidal Turbine}

As a high degree of similarity between the dynamic rotor properties was attained through the validation process, it was ascertained that the modelling approach and turbulence model implemented were of appropriate physical representation for a rotating bladed system; in recognition, the numerical schemes were adopted for the analysis of the ducted tidal turbine. By this means, in relation to studies that have endeavoured in modelling ducted tidal turbines $[4,5,28]$, this present study produces a more coherent representation of the configuration, both due to the accurate geometrical representation, and in the numerical modelling of general conservation and turbulence, hence better analysing the hydrodynamic occurrence of the fluid-structure interaction.

\subsection{Power Coefficient}

Upon implementation of the validated modelling techniques for the ducted turbine model, unique physical performance outcomes were displayed in representation of its high-solidity characteristics. Notably, the power coefficient TSR curve is relatively short spanning, with a TSR range of $1.00-2.50$ to achieve a $C_{P}$ decrease of 0.05 from the nominal TSR. Within this region, a maximum power coefficient range between 0.335 and 0.346 , at free-stream velocities of $1 \mathrm{~m} / \mathrm{s}$ and $7 \mathrm{~m} / \mathrm{s}$ respectively, was achieved, as illustrated in Figure 8 . Throughout the free-stream velocity variation, the power coefficient decrease is largely consistent postnominal TSR. The nominal TSR point, however, differs at distinctive velocities. At low free-stream velocities $\left(U_{\infty}=1 \mathrm{~m} / \mathrm{s}\right)$, the nominal TSR lies at 1.50 , whereas at higher velocities $\left(U_{\infty}=2.5-7 \mathrm{~m} / \mathrm{s}\right)$, the nominal TSR was found to shift to 1.75 . This variation in nominal TSR was found to be in consequence of the angle-of-attack $\left(\alpha_{A o A}\right)$ attained by the differing hydrofoils composing the blade.

As part of the work detailed in the thesis, Allsop [3] investigated the effect of the variation of chord-based Reynolds number on the hydrodynamic performance coefficients of the flatplate hydrofoil sections comprising the high-solidity blade utilising CFD. By considering a Reynolds number range of $1 \cdot 10^{6}$ to $1 \cdot 10^{7}$, a transition in the hydrofoil stall angle was depicted, increasing from an angle of $11^{\circ}$ to that of $14^{\circ}$, respectively. As the distinct flat-plate hydrofoil sections constitute the full blade, the effect of this stall angle transition is depicted in the power coefficient curve. At low TSR, the angle of attack at distinct elements within the blade is considerably high, inducing flow separation due to boundary layer detachment along the downstream surface, resulting in a region of the blade to experience stall. With an increase in TSR, the angle of attack decreases to the stall threshold, where the highest lift coefficient value is attained. Within this flow range, the boundary layer sticks to, and flows along, the section surface, resulting in the region to come out of stall. At this flow condition, optimal hydrodynamic parameters are induced, which characterises the criteria for peak power, occurring at optimal TSR. When this threshold is surpassed, a reduction in power is instigated in correlation to a steep linear decline in the lift parameter response of the flat-plate hydrofoil. The stall threshold is proportional to the chord-based Reynolds number, and hence, due to the shift in angle-of-attack, the optimal TSR correspondingly shifts to a higher TSR value at higher Reynolds numbers. Simulating more domain flow 


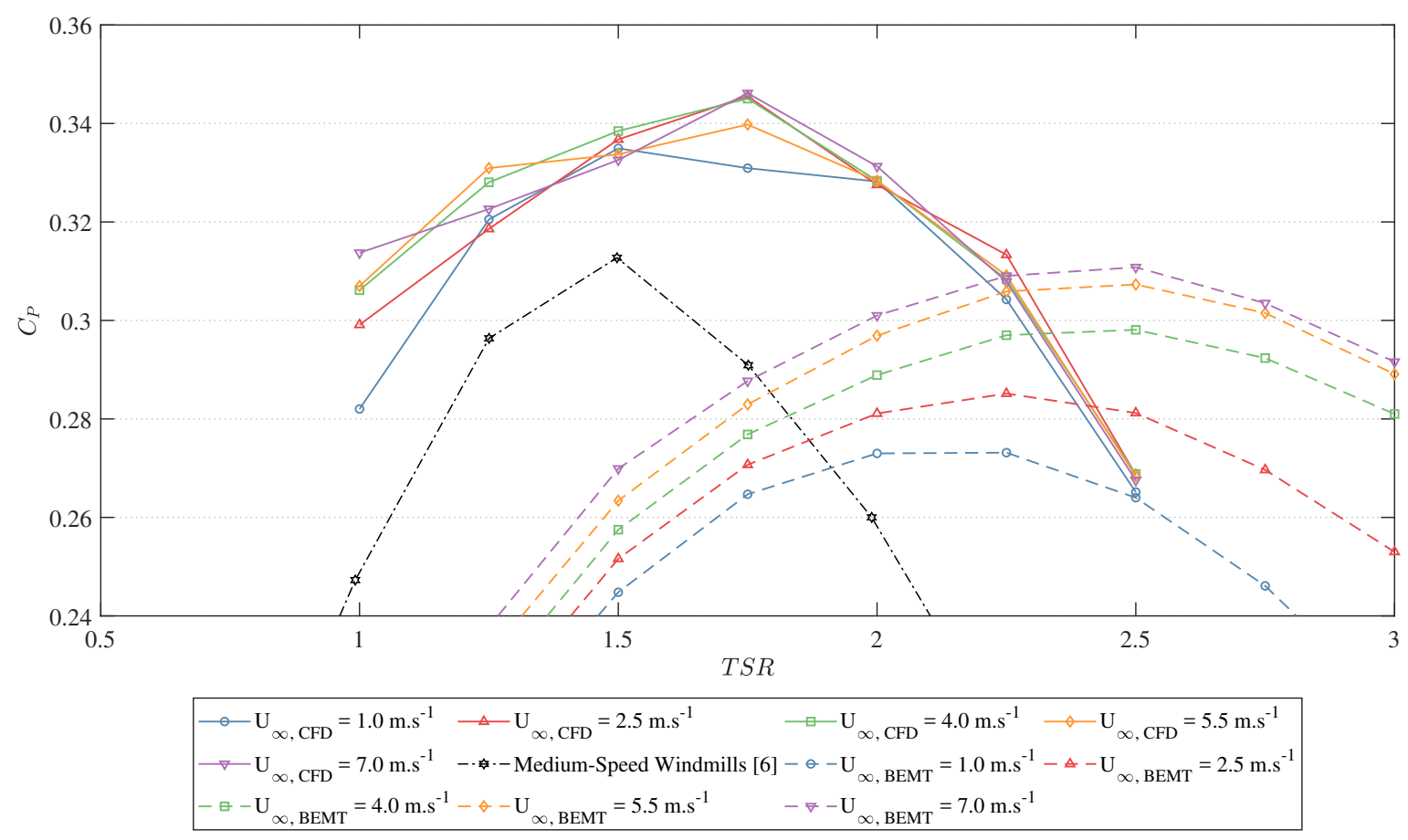

Figure 8: Evaluation of the mean ducted turbine power coefficient $\left(C_{P}\right)$ in relation to TSR

parameters would enable the identification of the definite TSR at which the blade is within the peak lift region, hence the point at which the degree of blade area within the stall region reaches tipping point. The physical value of this output, however, may be limited due to the sensitive nature of the rotating blade boundary layer, together with external influences from the turbulent and multi-directional nature of the tidal environment.

In accordance to the hydrodynamic performance characteristics, albeit a maximum power coefficient of 0.346 was attained, it may also be acknowledged that, due to the device area $\left(A_{d v c}\right)$ being considered, hence rotor \& duct, the power coefficient will increase once the power per rotor area $\left(A_{r t r}\right)$, rather than system area, is acknowledged. In this respect, the rotor radius is $80 \%$ that of the system, hence increasing the maximum power coefficient by a magnitude of 1.5625 to a nominal peak value of 0.541 . Due to the fact, however, that the power attained by the rotor is also disseminated from the fluid in contact with the duct, the entire system area is a more conservative approach to represent.

In comparison to BEMT modelling elaborated in [3], an areal discrepancy was acknowledged over the TSR range, and achieved moderately comparable figures at nominal TSR, with a discrepancy in evaluation of the maximum $C_{P}$, which ranges from 0.273 to 0.311 , at the free-stream velocities considered. This discrepancy may be elucidated by the dissimilarities in the hydrodynamic modelling of the two numerical techniques. Notably, the BEMT method considers the turbine blade as an array of discrete hydrofoil sections, considerably reducing the computational expense, where the hydrodynamic parameters of the blades are attained by means of two-dimensional CFD analyses. This approach, however, neglects any span-wise flow interactions along the blade length, together with omitting complex flow sep- 


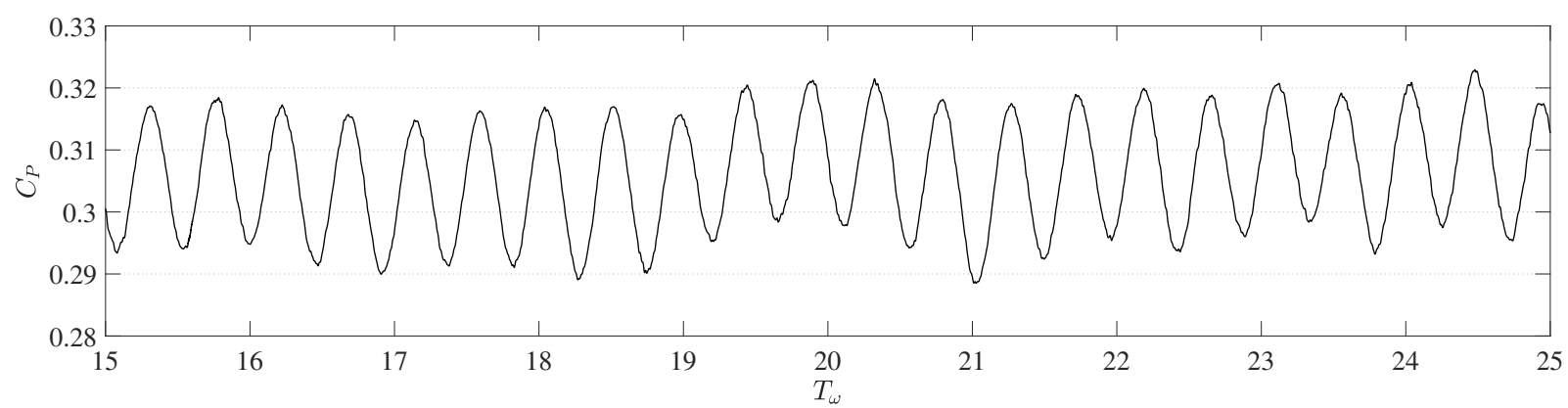

(a) $T S R 1.00$

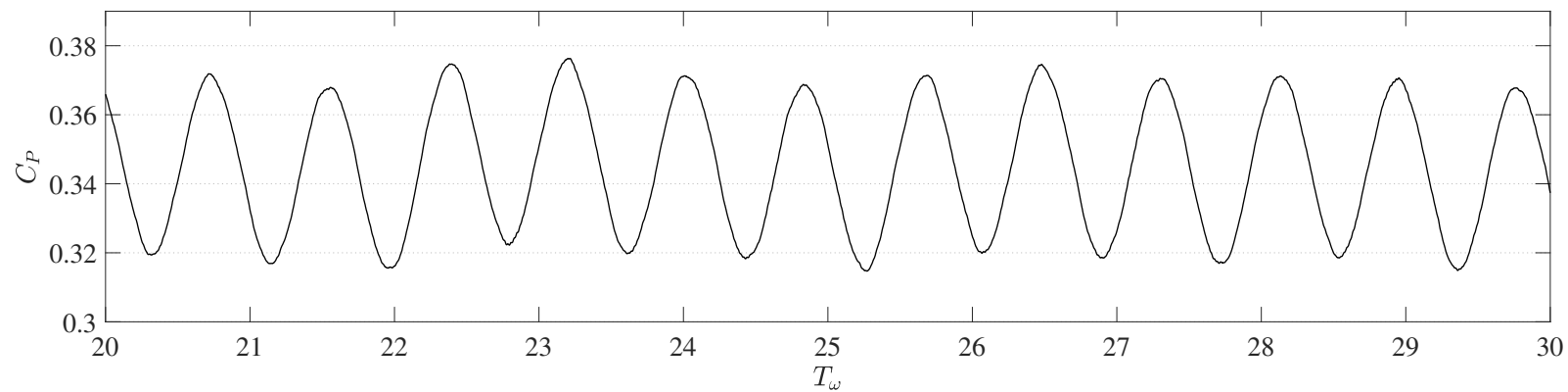

(b) TSR 1.75

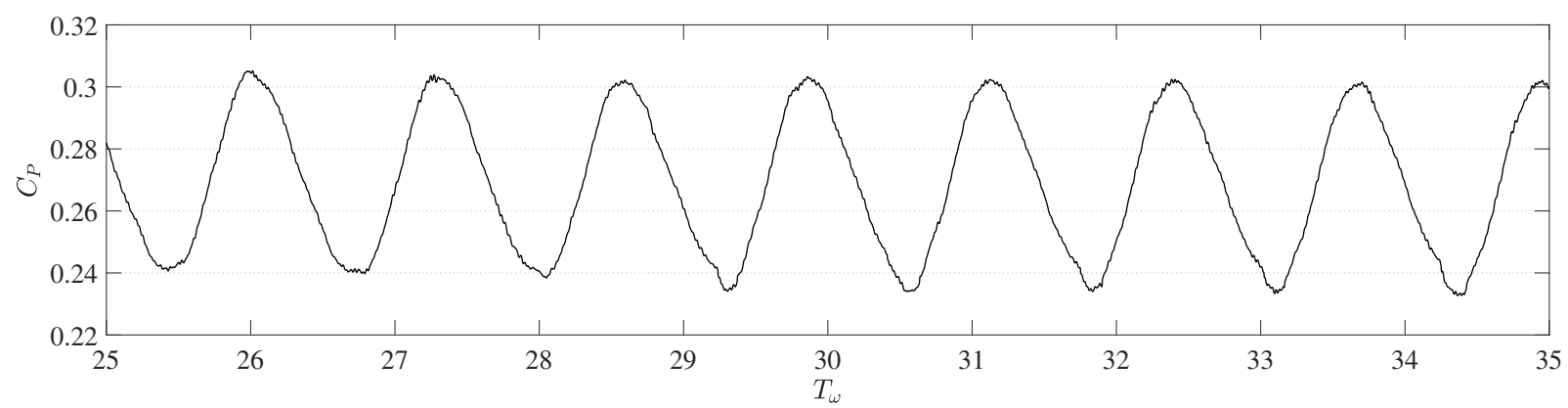

(c) TSR 2.50

Figure 9: Representation of the transient power coefficient output at $U_{\infty}=4.0 \mathrm{~m} / \mathrm{s}$ through turbine rotary periods $\left(T_{\omega}\right)$

aration at the open-centre blade tips, which are considered to be significant fluid dynamic features. The latter phenomenon is accounted for in BEMT by implementing the Prandtl tip loss factor, yet is better captured when utilising blade-resolved CFD. Albeit the drawbacks of the BEMT methodology, significantly accurate outputs are attained in consideration of the inputs provided within the numerical model contrived and the computational duration required.

Whilst presenting windmill findings in the technical memorandum, Betz [6] described a "medium-speed windmill" that attains maximum efficiency at a low TSR value within a short-spanning TSR curve. Potentially resembling the OpenHydro PS2 tidal turbine, a comparison was made between the reported findings and the CFD results, also illustrated 


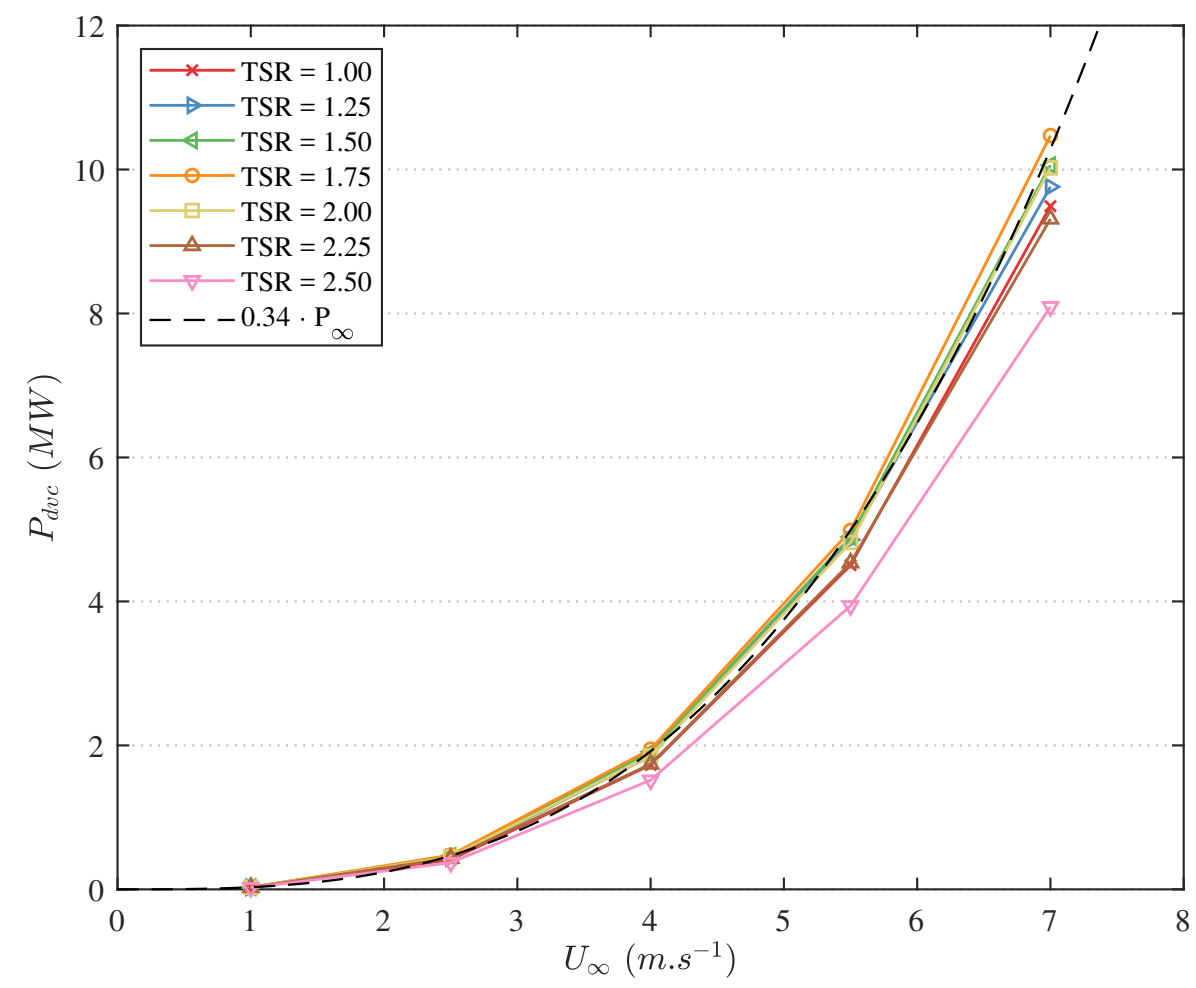

Figure 10: Evaluation of the ducted turbine mechanical power $\left(P_{d v c}\right)$ in relation to free-stream velocity $\left(U_{\infty}\right)$ at distinct TSRs

in Figure 8. With a peak windmill power coefficient of 0.313 at a nominal TSR of 1.50, good agreement was acknowledged in the parameter trend when compared to the ducted high-solidity turbine system.

In continuation, to comprehend the physical representation of the power generated by the turbine, Figure 10 illustrates the variation in the power output with the velocity of the free-stream. In comparison to the theoretical representation of a power curve, the peak power coefficients at nominal TSR $(T S R=1.75)$ at varying velocities match well with a mean power coefficient of 0.34 . Further to the power curve, the variation in the torque coefficient $\left(C_{Q}\right)$ induced on the turbine is illustrated in Figure 11. Similar areal discrepancies are attained in comparison to BEMT and the Betz curve, yet, albeit the differences, the key similarity between the comparisons is the trend at which the torque varies.

In consideration of the substantial correspondence between the power \& torque coefficient performance curves displayed within the CFD results, BEMT outputs, and windmill (experimentation) findings, the applicability of full-scale analysis by means of the methodology implemented was acknowledged. The total discrepancy, however, both in comparison to windmill findings and BEMT outputs, may be a result of the over-sensitivity of the implemented turbulence model physics. This, ultimately, may overestimate the pressure distribution on the wall-bounded conditions, and hence, mechanical power transition due to 


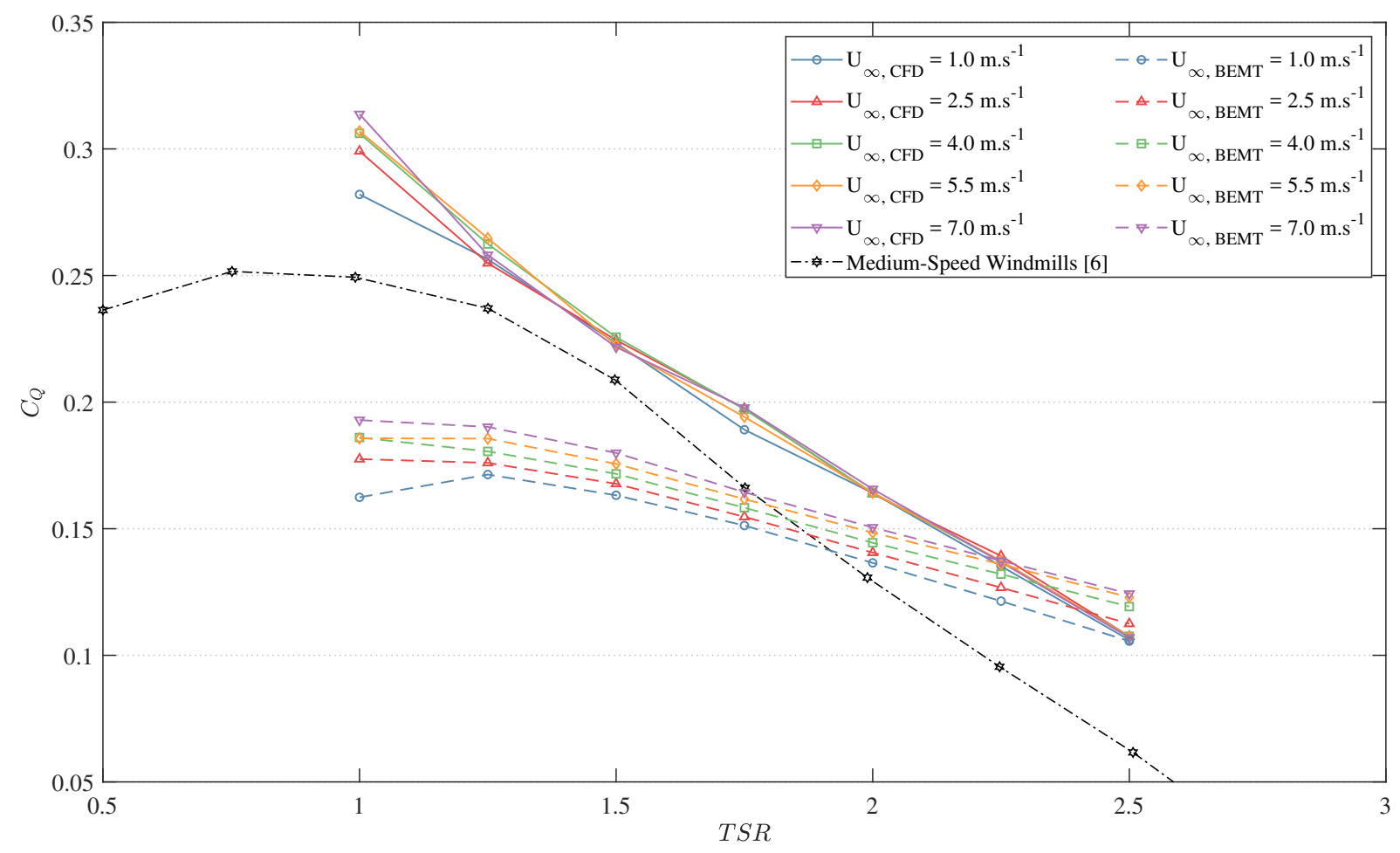

Figure 11: Evaluation of the ducted turbine torque coefficient $\left(C_{Q}\right)$ in relation to TSR

the shear flow induced at the surfaces of the blades; further analyses utilising differing turbulence models may be required to ascertain this outcome. In definition of the parametric outcomes, the imperative of executing both CFD and BEMT methodologies when analysing turbine performance is exemplified. A denomination of the induced physical effects are more appropriately identified by means of a simplified model, and may be of assistance in understanding the fluid dynamic outcomes of higher complexity analyses, such as that described within this work, due to the model capacity in capturing multiple coupled effects, such as lateral flow interactions down the blade length and temporal fluctuations in the rotor performance, as depicted in Figure 9, where the individual physical phenomena inducing the hydrodynamic behaviour are less obvious.

\subsection{Thrust Coefficient}

The implementation of a duct along the perimeter of a turbine subsequently increases the area onto which an external force may be induced, potentially bringing about an intensification in physical bluff-body conditions. In effect, the combined drag induced upon the system becomes of particular interest.

Illustrated in Figure 12, the variation in thrust coefficient $\left(C_{T}\right)$ with TSR and freestream velocity is presented both in terms of the entire system, and in terms of the physical components: rotor and duct. As an entire system, a substantially high thrust coefficient is attained at low TSR, a mean value of 1.17, which decreases to a mean of 0.70 at high TSR. The gradient acts in a polynomial manner to nominal TSR, where the mean thrust coefficient 
is precisely 1.00, after which the gradient acts linearly. Observed from the component curves, this considerable discrepancy in drag is largely consistent upon both the duct and the rotor, with a mean allocation of the global drag of approximately $32 \%$ and $68 \%$, respectively. The rotor hence succumbs to roughly two-thirds of the global drag, whilst the duct succumbs to one-third; this allocation is somewhat relative to the areas of the duct and rotor, being $36 \%$ and $60 \%$ of the system area, respectively. In comparison to BEMT, which solely considers the drag on the rotor, a significant discrepancy is attained between the two methodologies, albeit exhibiting a moderately similar diminishing $C_{T}$ trend.

In effort of establishing the source of the substantial drag on the rotor, the static pressure coefficient $\left(C_{P r}=\frac{P_{s}}{\frac{1}{2} \rho U_{\infty}^{2}}\right)$ distributions within the domain at the vicinity of the rotor were qualitatively analysed. Principally, the induction of axial drag upon a structure is a straightforward concept; pressure upstream and suction downstream of the structure induces this outcome. Illustrated in Figure 13, at low TSR, the static pressure upstream and the suction downstream of the rotor are substantial. Acknowledging the flow-rate through the duct, at low TSR, the flux is comparatively low, as illustrated in Figure 14. As a result, as the freestream comes into constant contact with the structure, fluid accumulates at the inlet of the duct, upstream of the rotor, hence increasing the static pressure in this region. Furthermore, due to the high-solidity of the rotor, insufficient fluid is permitted to flow through the duct, hence creating a region of low pressure. The duct sustains this variation in pressure as the upstream duct section confines the flow in the vicinity of the rotor, disallowing its leakage, hence inducing a pressure build-up, whilst, directly downstream of the rotor, the duct disallows the free-stream from re-energising the wake, hence sustaining the low pressure zone. As TSR increases, the flow rate through the duct proportionally increases, hence decreasing the static pressure difference fore and aft of the rotor, subsequently reducing drag on both the rotor and the duct; BEMT does not incorporate this fluid dynamic induction within its methodology, hence expounding the discrepancy observed. The variation in mass flow-rate through the turbine, which attains a mean coefficient of 0.680 at low TSR, and increases to a mean of 0.715 and 0.805 at nominal and high TSR, respectively, inversely proportional to the resultant thrust, indicates the substantial influence in the total drag induced upon the entire structure. In consequence to the extensive capacity in mass-flow rate development, due to its high-solidity properties, as TSR is further increased, a negative pressure difference is eventually induced, resulting in the turbine to act as a propeller.

In addition to the system drag principally comprising of the rotor drag, the force on the duct, apart from also being effected by the sustained pressure at the rotor, is dependent on the fluid-structure interaction at the profile of the duct structure. As the external fluid flow is in contact with the entire structure, a significant degree of static pressure builds up at the walls of the duct inlet. This consequently affects the incoming flow, as the far-flow in the direction of the system takes the path of least resistance, and hence bypasses the duct inlet, and flows along the duct outer surface without any interaction with the internal structure. Fluid in the vicinity of the inner duct ridges, however, impacts the internal structure, and interacts with the duct by flowing along the inner duct curvature, either against the freestream, in an upstream direction away from the rotor, or with the free-stream, towards the 

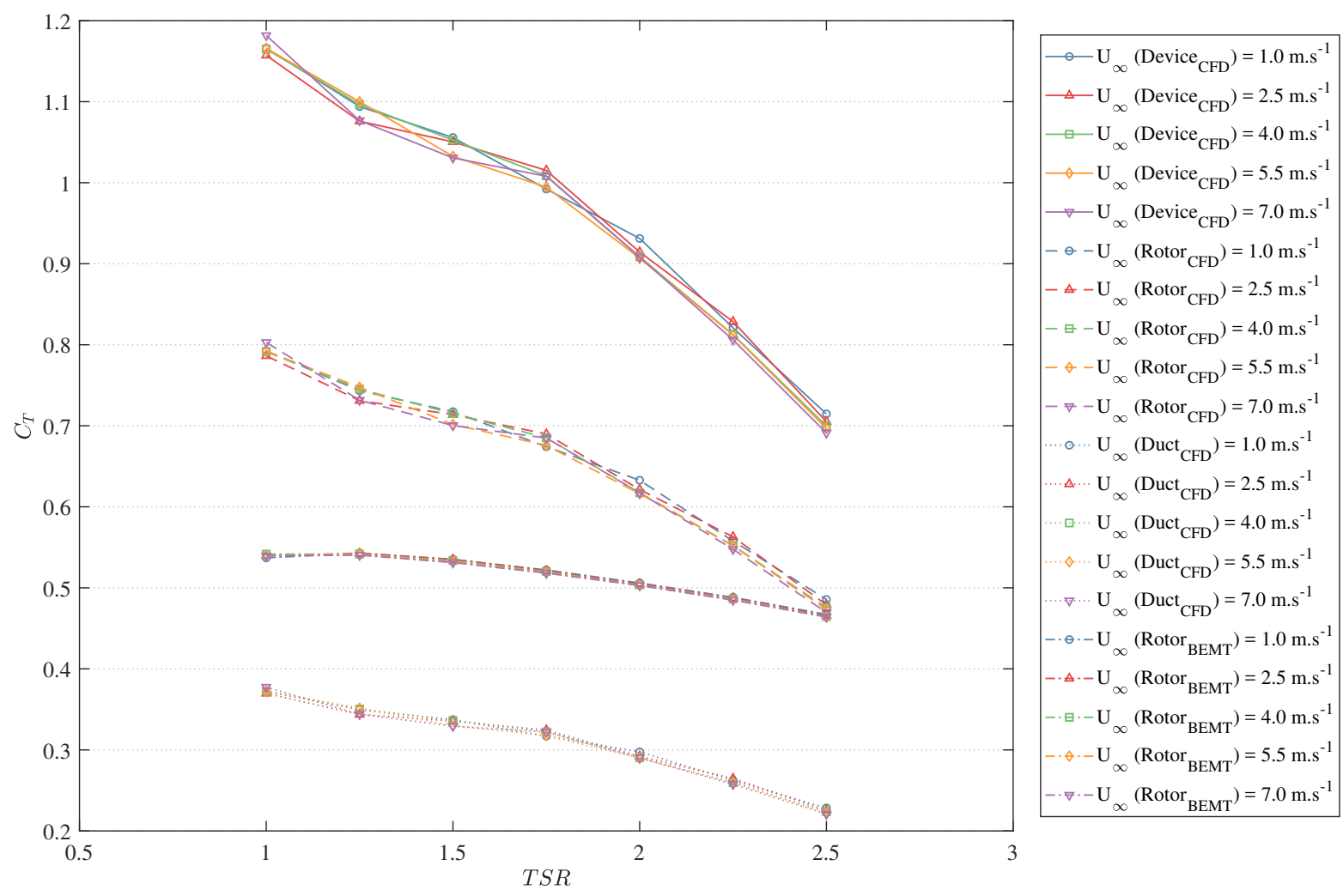

Figure 12: Evaluation of the ducted turbine thrust coefficient $\left(C_{T}\right)$ in relation to TSR

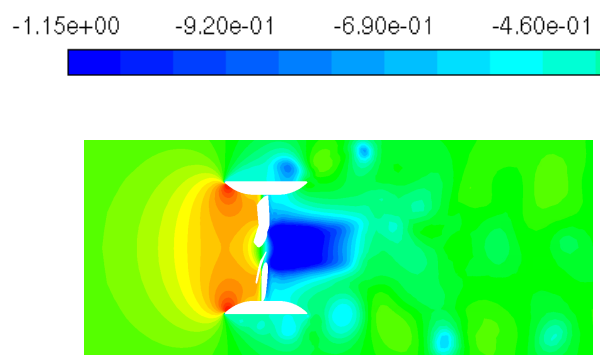

$0.5 T_{\omega}$

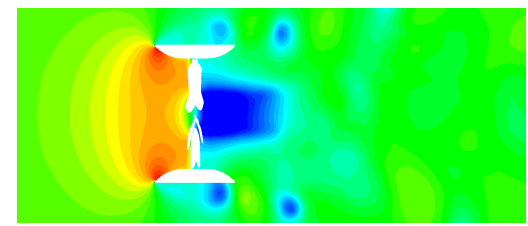

$1.0 T_{\omega}$

(a) $T S R=1.00$

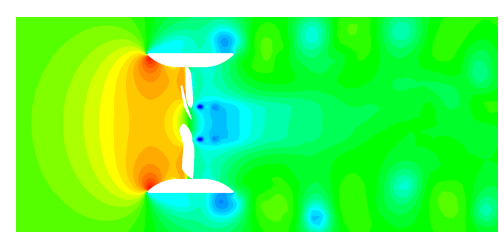

$0.5 T_{\omega}$

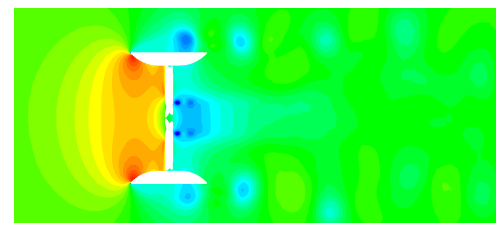

$1.0 T_{\omega}$

(b) $T S R=1.75$

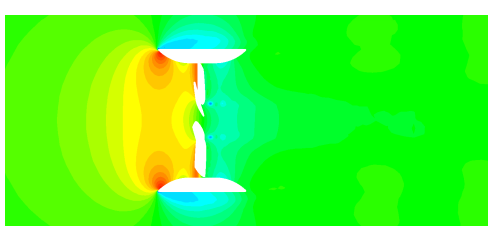

$0.5 T_{\omega}$

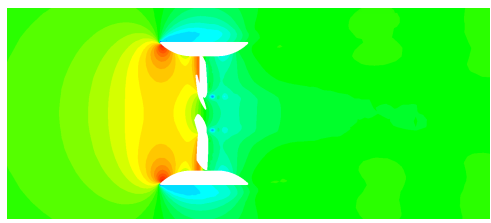

$1.0 T_{\omega}$

(c) $T S R=2.50$

Figure 13: Illustrative top-view representation of the pressure coefficient $\left(C_{P r}\right)$ within the turbine domain at low, nominal, and high $T S R$ at mean-average output; $U_{\infty}=4 \mathrm{~m} / \mathrm{s}$ 


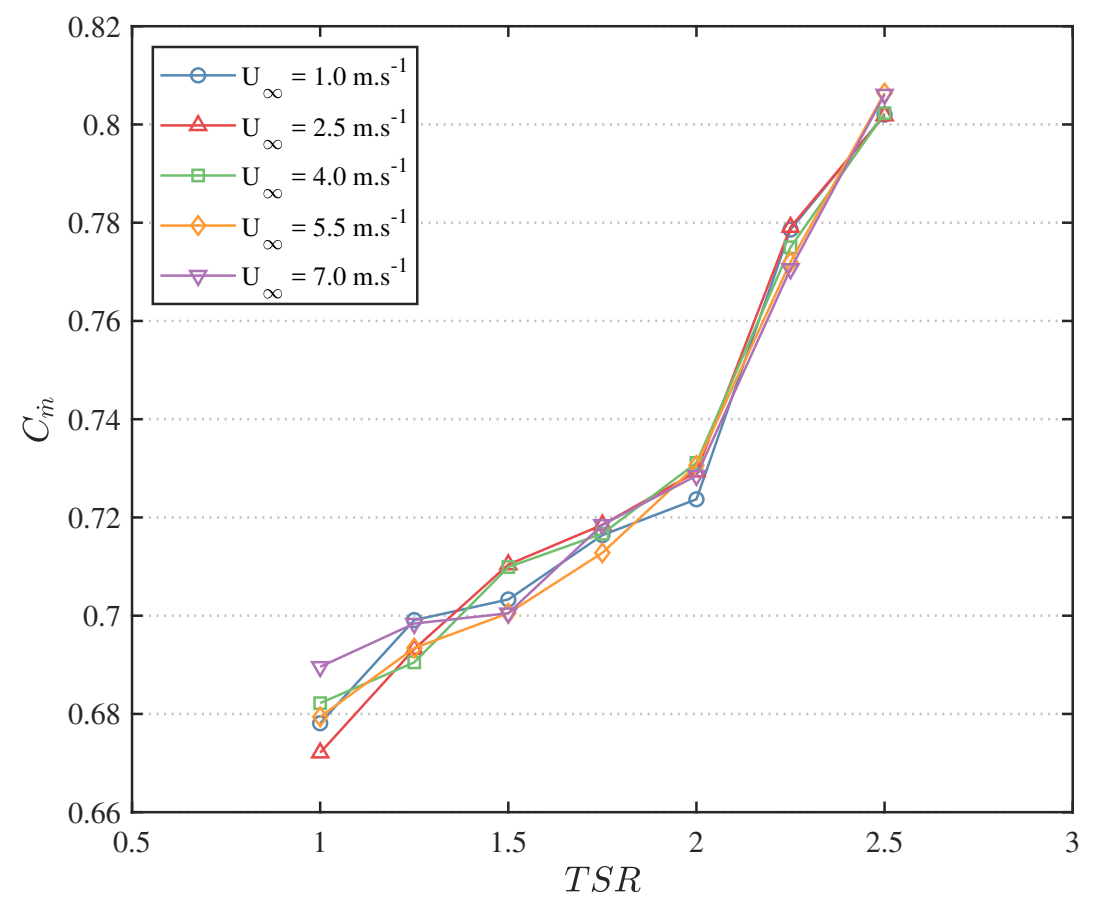

Figure 14: Evaluation of the ducted turbine mass-flow rate coefficient $\left(C_{\dot{m}}\right)$ in relation to TSR

rotor, illustrated in Figure 15a, both of which towards regions of lower pressure. Physically, along the perimeter of the duct, the point at which this occurs is the point of pressure stagnation, where static pressure is the highest, as velocity is nullified, and hence, induces significant drag; in this system, the stagnation point is induced, on average, at $\sim 0.94 R_{d c t}$.

\subsection{Static Pressure on Duct Ridges}

The fluid upstream of the stagnation point induces a unique phenomenon, due to the flow being forced back up the duct, rather than through the turbine. Once the flow surpasses the duct ridges, it interacts with the free-stream; due to the distinct velocities of the two flows, this induces a resultant, deflected flow misaligned to the free-stream, and hence, misaligned to the surface of the duct, resembling flow spillage. Similar to flow around a geometrical bend, substantial flow separation is induced, resulting in a region of re-circulating fluid, known as a separation bubble, within which rotational flow and turbulent mixing occur upon the duct surface, creating a low pressure zone. This concept is illustrated in Figure 15b, where the static pressure along the inner upstream duct surface is pressurised due to the inlet flux. Flux escapes upstream of the stagnation point, and results in a consistent measure of negative static pressure induced along the entire length of the outer duct, at all TSRs, implementing a degree of suction, induced by the re-circulating zone.

This phenomenon occurs at all TSRs modelled, yet the physical size of the re-circulation zone is seen to vary in an inversely proportional manner to the TSR of the rotor. As a result of the substantial static pressure within the duct at low TSRs, due to the lower mass-flow rate 
through the rotor, the flux of the fluid diverted upstream of the rotor is higher than that at higher TSRs. For this reason, the velocity resultant at the interaction with the free-stream is at a more obtuse angle to the surface of the duct than that at higher TSRs, creating a larger vortex zone. In addition, along the surface of the outer duct, the skin friction coefficient is minute, hence depicting that the re-circulation zone is incapable of completely reattaching. In consequence, the re-circulation zone is physically unstable, ensuing in the shedding of the vortices generated within. Therefore, the external flow interacting with the duct will instigate vorticial rotational motion via the region of re-circulating fluid along the length of the duct, which is then shed at the duct outlet.

\subsection{Vortex Shedding}

Aft of the rotor, within the duct, the downstream turbine wake does not expand concomitant to the variation in the duct outlet geometrical profile, yet expands at an angle more acute than the gradient of the inner duct contour. This signifies that the flow refrains from attachment when flowing towards the outlet rim, hence providing evidence of boundary layer separation, decreasing the duct efficiency. In addition, slight flow separation is induced within the fluid region between the wake and the duct due to the free shear layer at the boundaries of the rotor wake, as illustrated in Figure 16. The distinct vortices, formed by the re-circulating fluid region along the circumference of the duct, and shed at the duct outlet as ring vortices, as illustrated in Figure 13, are transported within the turbine wake from this location. At low TSRs, the wake demonstrates a highly disorganised flow structure with turbulent intermixing, as illustrated in Figure 17. The large volume of the re-circulating zone induces substantial rotational motion within the created vortices, resulting in vortex shedding at comparatively high rotational kinetic energy; the high-energy vortices commingle with the low-energy wake, creating a chaotic wake. With an increase in TSR to nominal TSR, a consistent ring vortex stream is identified in the wake. The vortex effect diminishes with an increase in TSR, due to the reduction of the re-circulating fluid zone and, hence, rotational kinetic energy. As the wake contains a higher degree of energy, this results in a more streamline wake at high TSRs; flow irregularity is nonetheless present in the wake, yet is comparatively minute, and is solely transported within until dissipation.

Correspondent to the vortex shedding, an analysis regarding the vortex effect on the axial drag force developed was considered by superimposing a sinusoidal function upon the global drag of the system in an effort to attain the frequency of the fluctuation. As a result, the vortex shedding frequency was found to be linearly proportional to the free-stream velocity, and, hence, Reynolds number $\left(R e_{L}\right)$, as illustrated in Figure 18, in agreement with Strouhal's law; the shedding frequency is, therefore, solely dependent on the free-stream velocity of the domain. The quantity of the vortices shed per rotor rotation, however, act in an opposite manner, being independent of the free-stream velocity, yet dependent on the TSR. Within that attribute, a mean of 2.15 and 0.83 vortices per rotation are shed at low TSR and high TSR, respectively. In attaining a Strouhal number $(S t)$ for the system, the duct length $\left(L_{d c t}\right)$ was utilised as the Strouhal characteristic length $\left(L_{S t}\right)$, hence resulting in a constant mean global Strouhal number of 0.558 for the ducted turbine system. For this reason, vortex shedding may have a significant influence on the system, where the potential 


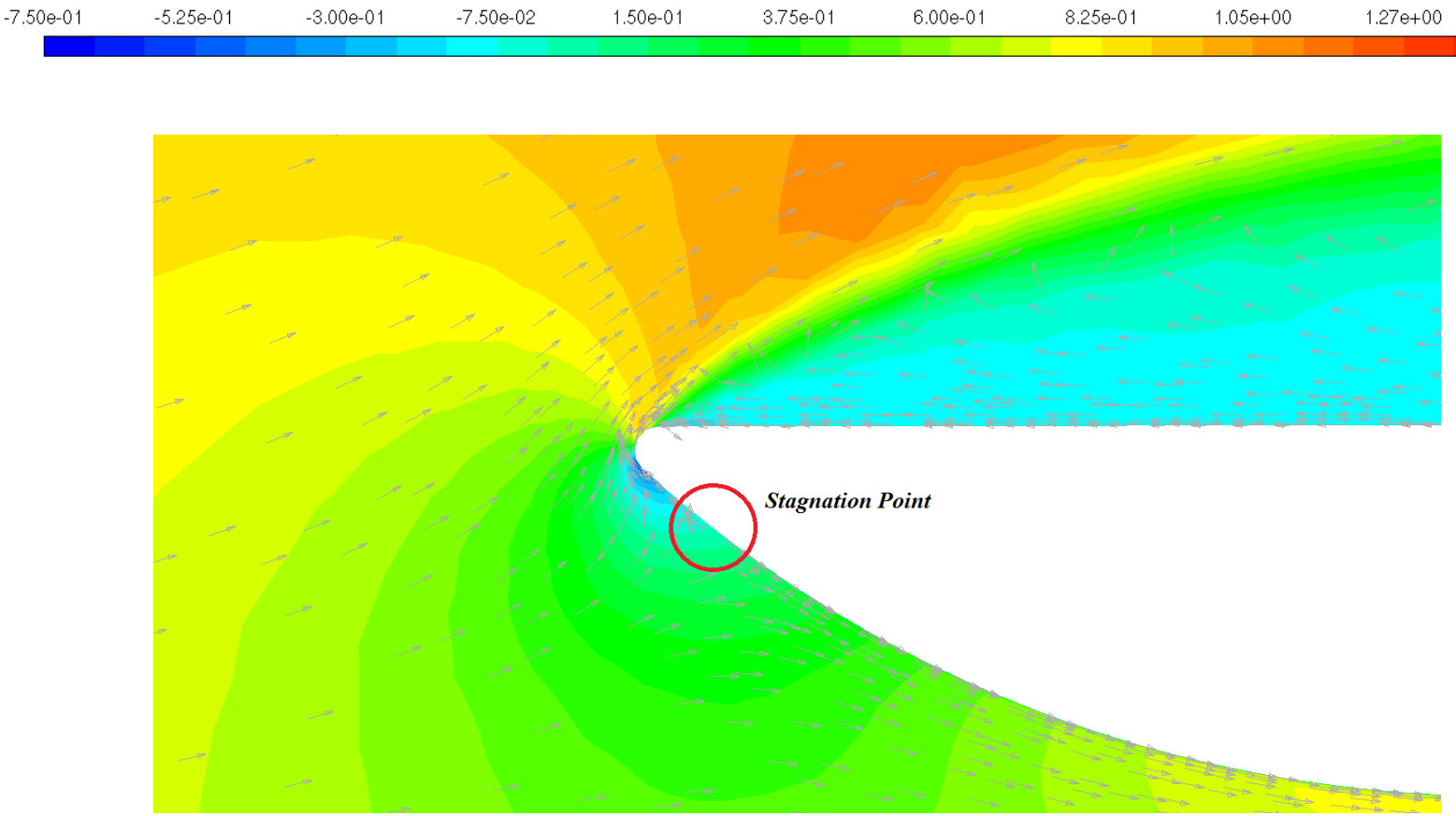

(a) Axial velocity coefficient $\left(\bar{u}_{z}^{*}\right)$ illustration of stagnation point on inner curved duct surface, and re-circulation zone on outer straight duct surface; $T S R=1.75$

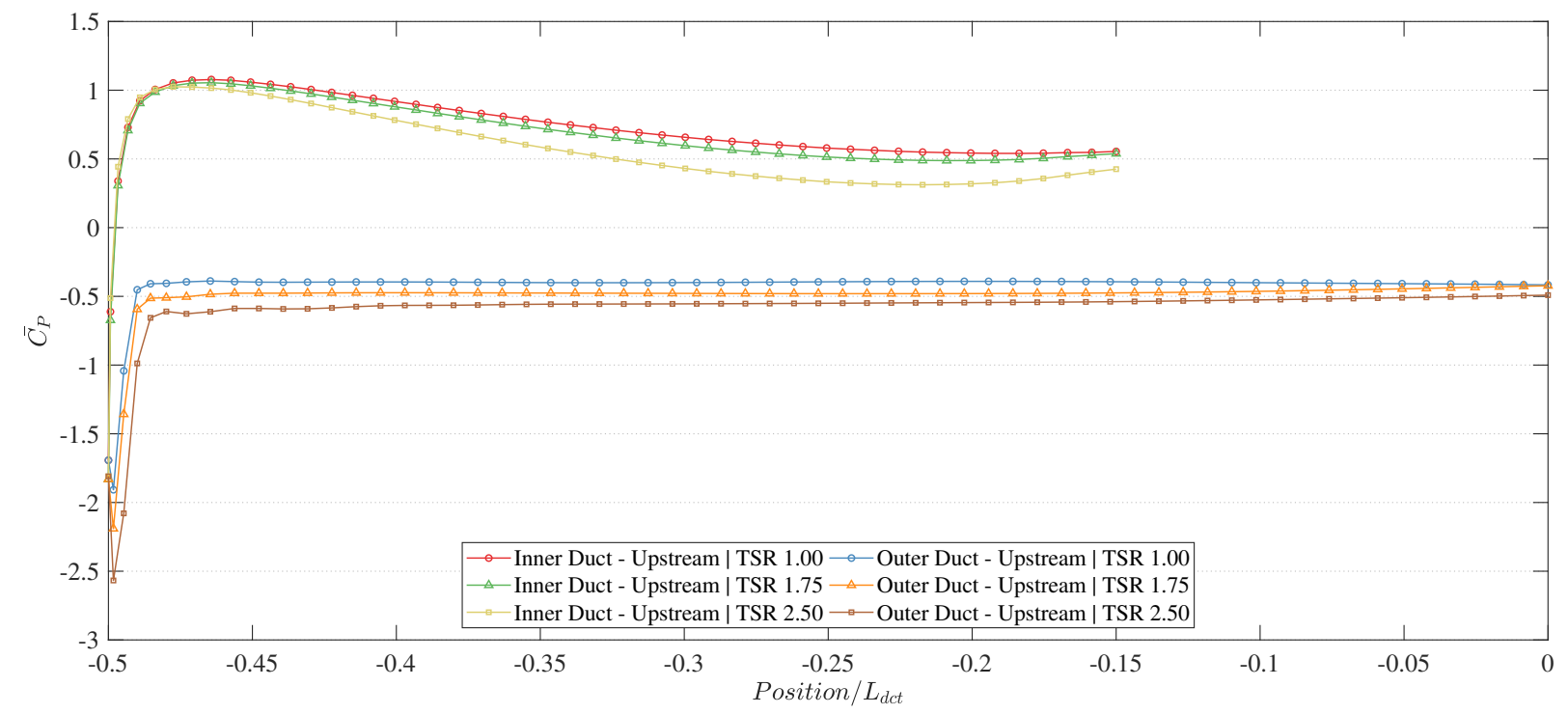

(b) Pressure coefficient $\left(C_{P r}\right)$ values at the inner curved and outer straight duct sections upstream of the rotor $\left(-0.5 \leqslant \frac{z}{L_{d c t}} \leqslant 0\right)$

Figure 15: Representation of the dynamics at the inner curved and outer straight geometry of the duct inlet; $U_{\infty}=4 \mathrm{~m} / \mathrm{s}$

presence of fatigue-inducing structural phenomena, such as vortex-induced vibrations (VIVs) 


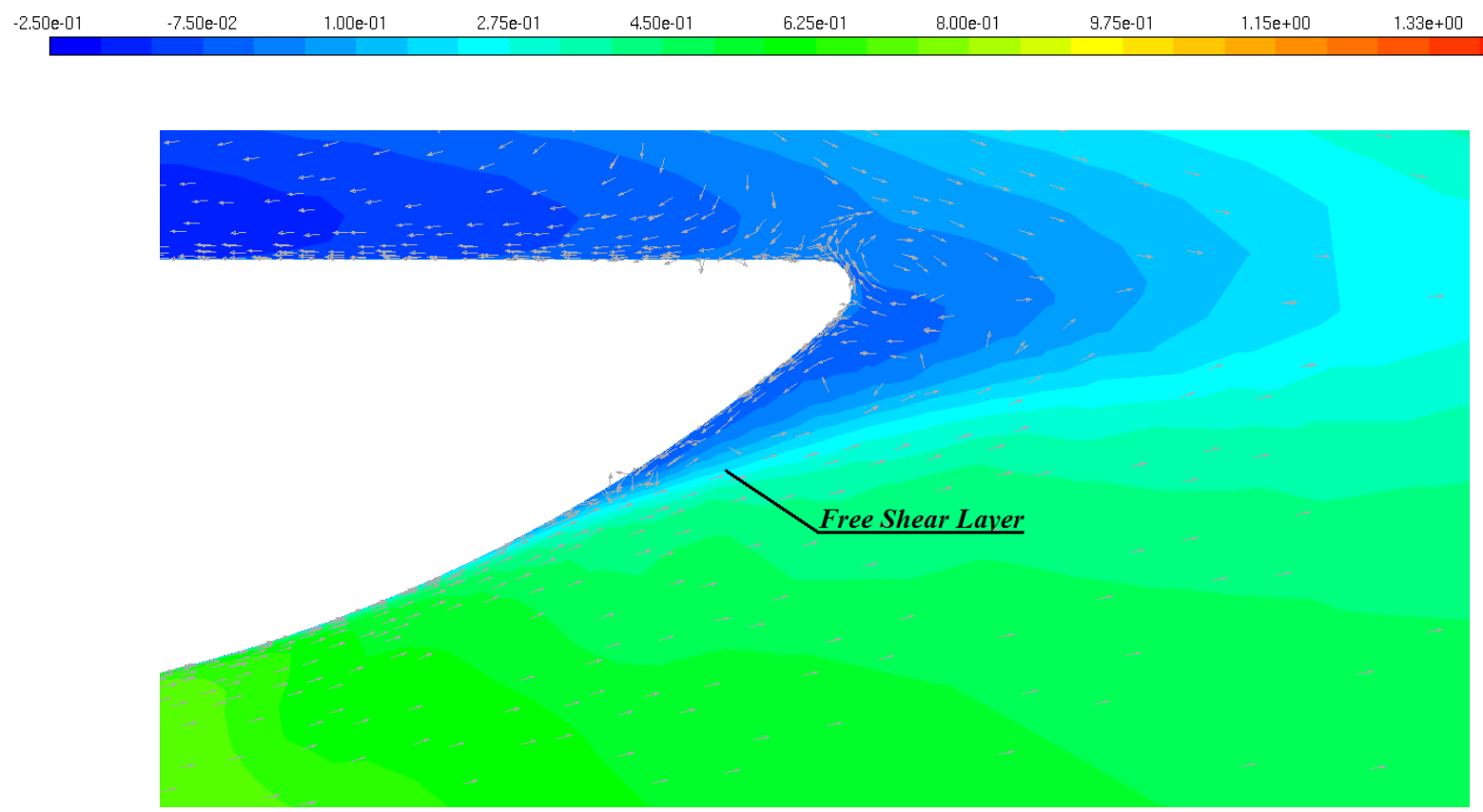

Figure 16: Axial velocity coefficient $\left(\bar{u}_{z}^{*}\right)$ illustration of the free shear layer at the duct outlet; $U_{\infty}=$ $4 \mathrm{~m} / \mathrm{s}, \mathrm{TSR}=1.75$

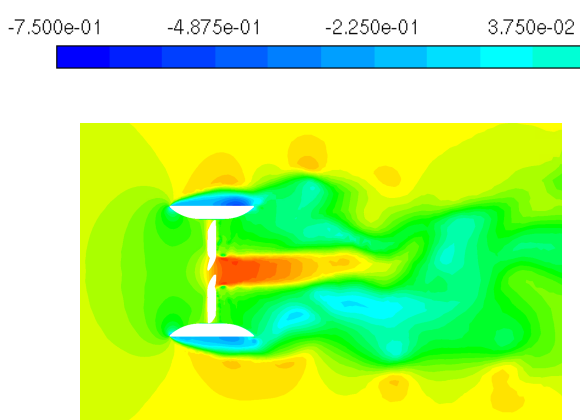

$0.5 T_{\omega}$

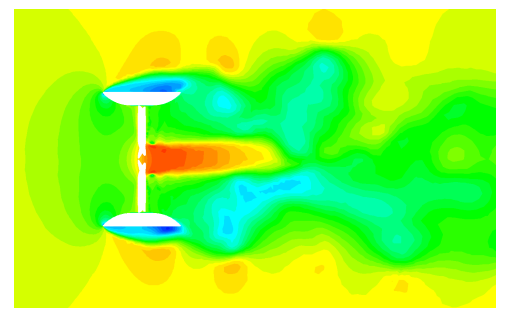

$1 T_{\omega}$

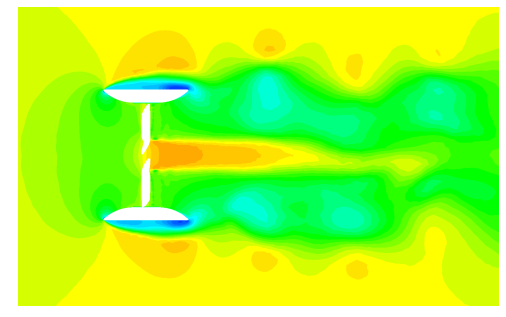

$0.5 T_{\omega}$

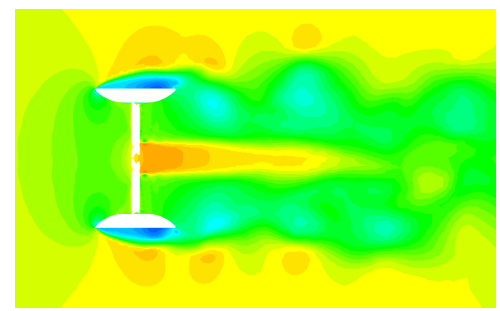

$1 T_{\omega}$

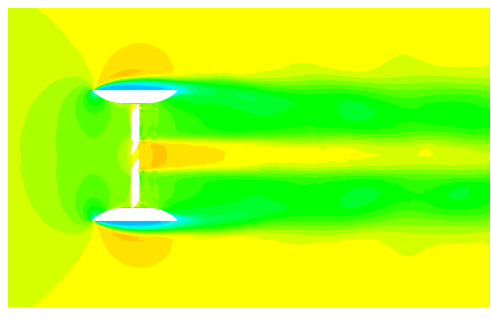

$0.5 T_{\omega}$

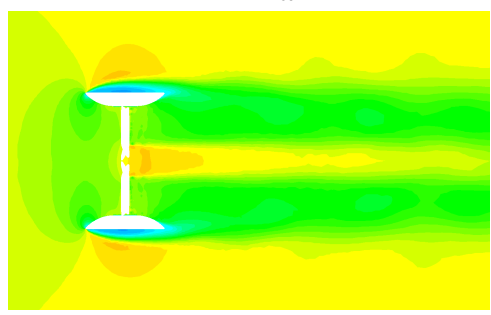

$1 T_{\omega}$
(a) $T S R=1.00$
(b) $T S R=1.75$
(c) $T S R=2.50$

Figure 17: Illustrative top-view representation of the axial velocity coefficient $\left(\bar{u}_{z}^{*}\right)$ within the turbine domain at low, nominal, and high $T S R ; U_{\infty}=4 \mathrm{~m} / \mathrm{s}$ 


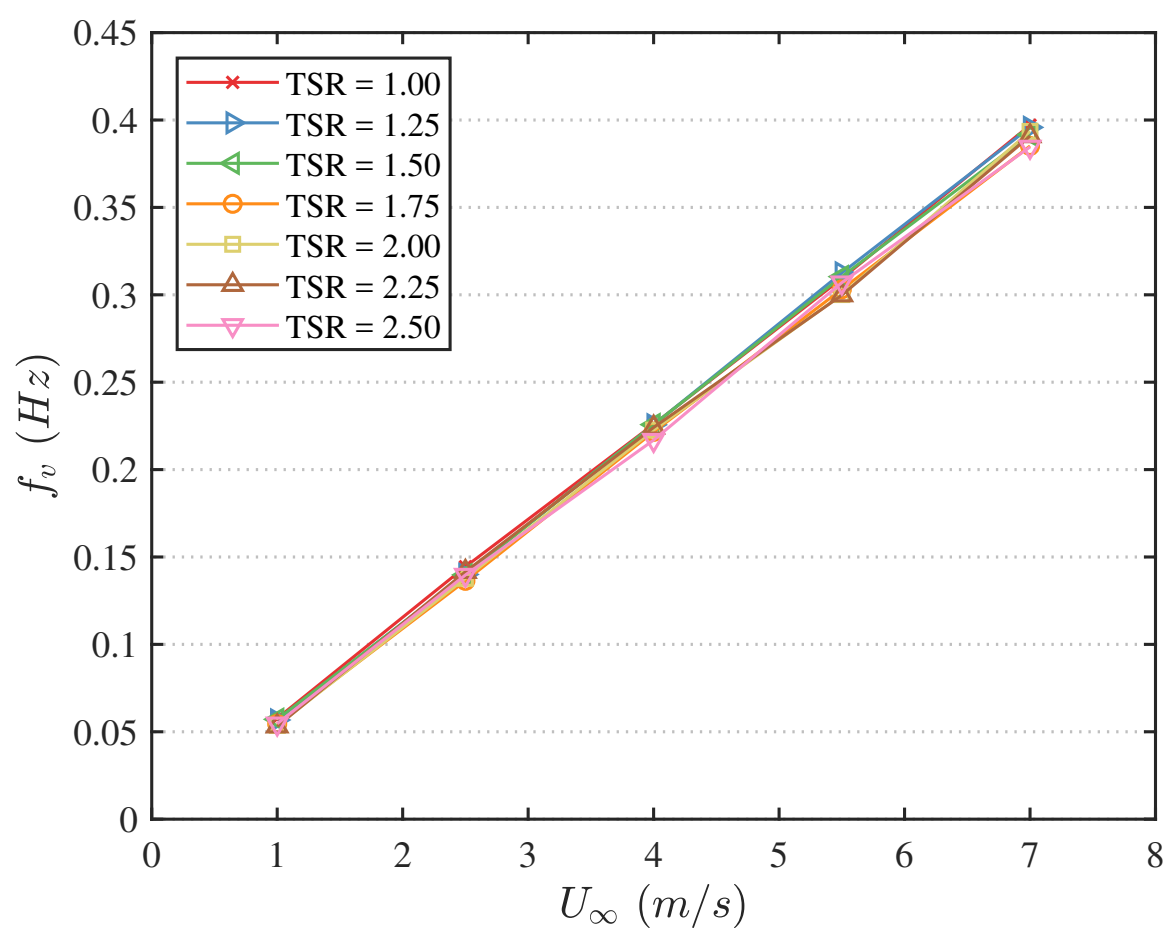

Figure 18: Evaluation of the vortex shedding frequency $\left(f_{v}\right)$ in relation to free-stream velocity $\left(U_{\infty}\right)$ at distinct TSRs

on its foundation, together with fluttering at the blades, become a consequential factor to the dynamics of the system.

\subsection{Blade Analysis}

Further to analysing the static pressure on the duct, in effort of acknowledging the hydrodynamic impact on the blades, the static pressure on distinct sections along the radius of the rotor was considered. Figure 19 illustrates the variation in pressure coefficient at nominal TSR in five physical iterations on the blade, from near-tip $\left(r_{r t r} / R_{r t r}=0.3633\right)$ to near-duct $\left(r_{r t r} / R_{r t r}=0.9300\right)$, to quantitatively extract the static pressure distribution along the rotor radius.

The distribution of static pressure along each section of the blades was found to be largely consistent with each other. With a pressurised side on the upstream surface of the blade, the stagnation point, at higher radii, is precisely at the leading edge of the blade, which shifts inwards along the pressurised surface at lower radii due to the variation in angle-of-attack. Attributable to the profile of the blade, a flat-plate with rounded edges, the acceleration of the flow around the leading edge induces a separation point at the downstream (suction) surface. Separation occurs at the point where the rounded edge joins the flat edges. This aspect is attributable to flat-plates with rounded edges; along the upstream (pressurised) surface, a pressure depression is induced, yet is sustained as a result of the external flow from the inlet of the duct. 


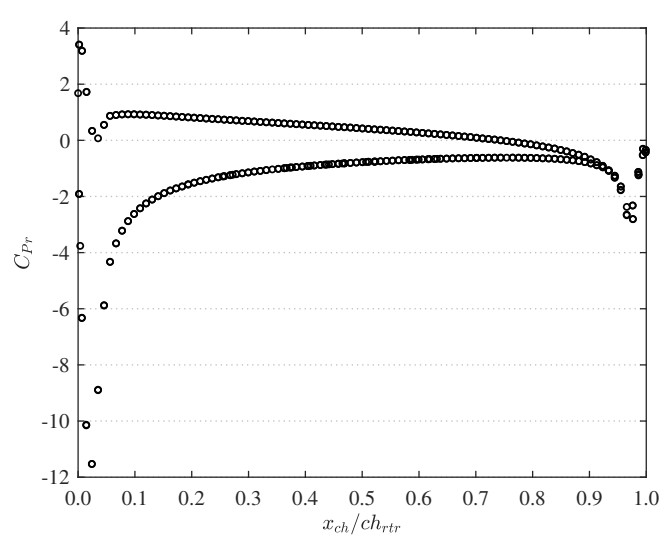

(a) $r_{r t r} / R_{r t r}=0.9300$

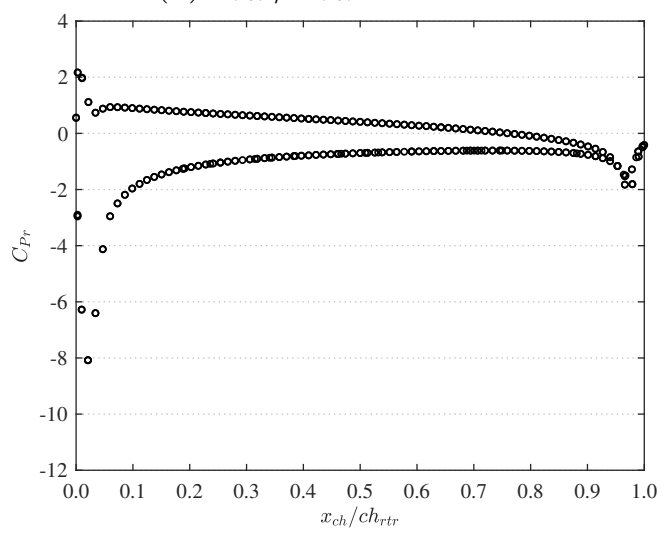

(c) $r_{r t r} / R_{r t r}=0.6466$

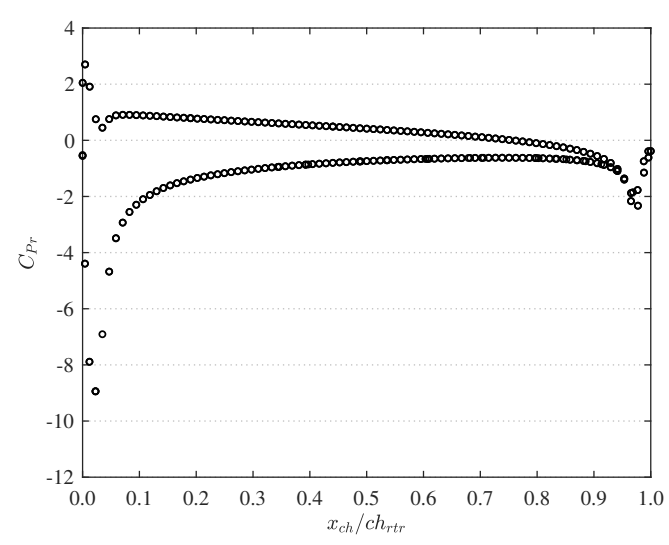

(b) $r_{r t r} / R_{r t r}=0.7600$

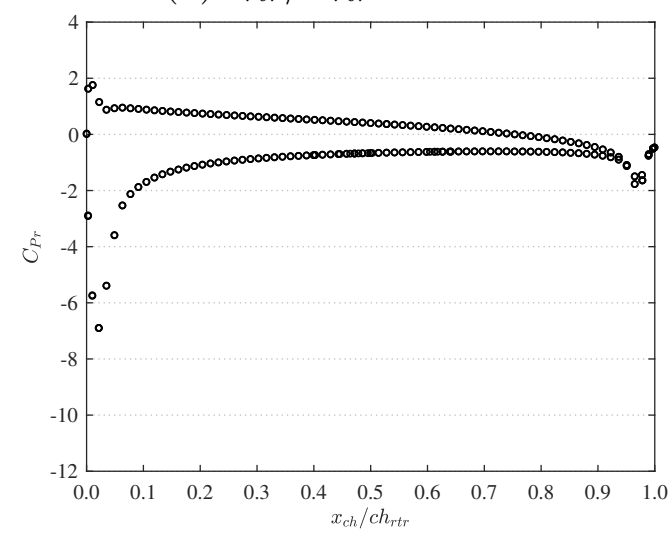

(d) $r_{r t r} / R_{r t r}=0.5333$

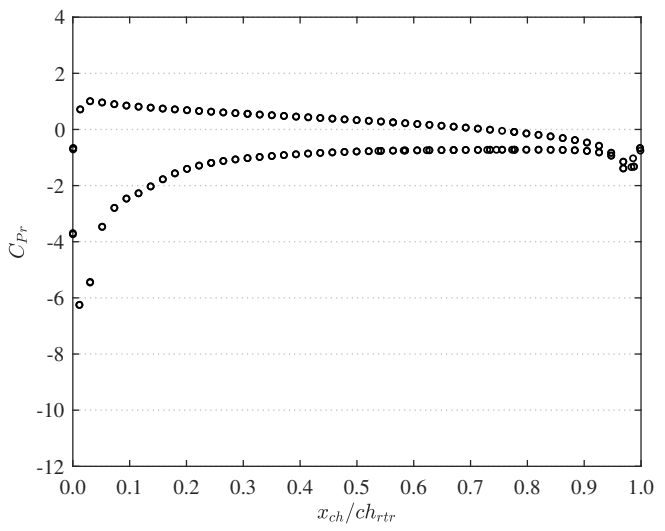

(e) $r_{r t r} / R_{r t r}=0.3633$

Figure 19: Plots of pressure coefficient $\left(C_{P r}\right)$ along the blade chord ratio $\left(x_{c h} / c h_{r t r}\right)$ at distinct rotor radius ratios $\left(r_{r t r} / R_{r t r}\right)$ at $U_{\infty}=4.0 \mathrm{~m} / \mathrm{s}, T S R=1.75$

Suction is dominant throughout the surfaces of the blades, withal at the upstream pressurised surface, where pressure decreases along the chord length, accentuating the inefficacy of the blade profile. A secondary pair of separation points are induced at the locations where the trailing rounded edge joins the flat plate, due to the sudden change in geometry, and 

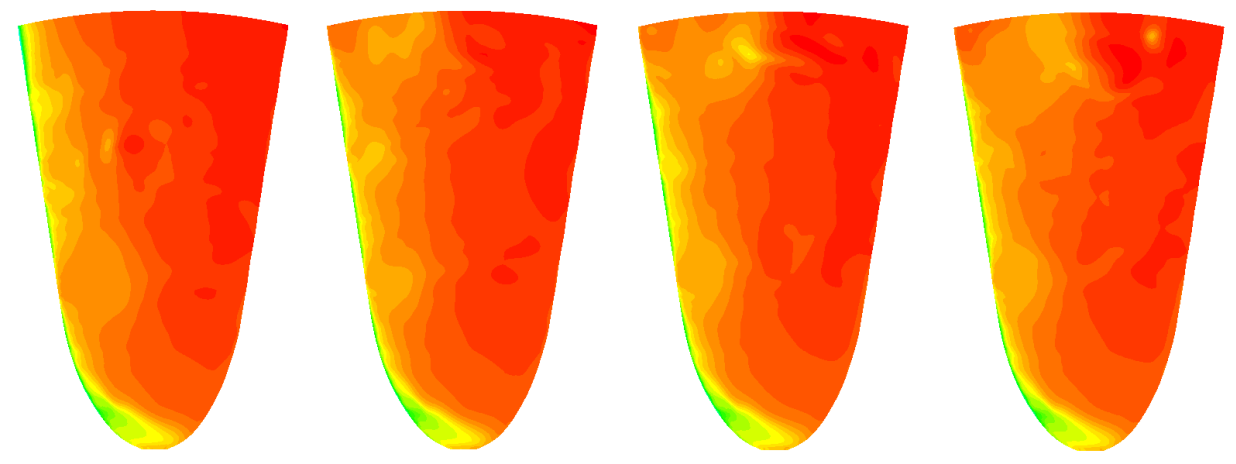

(a) $T S R=1.00$
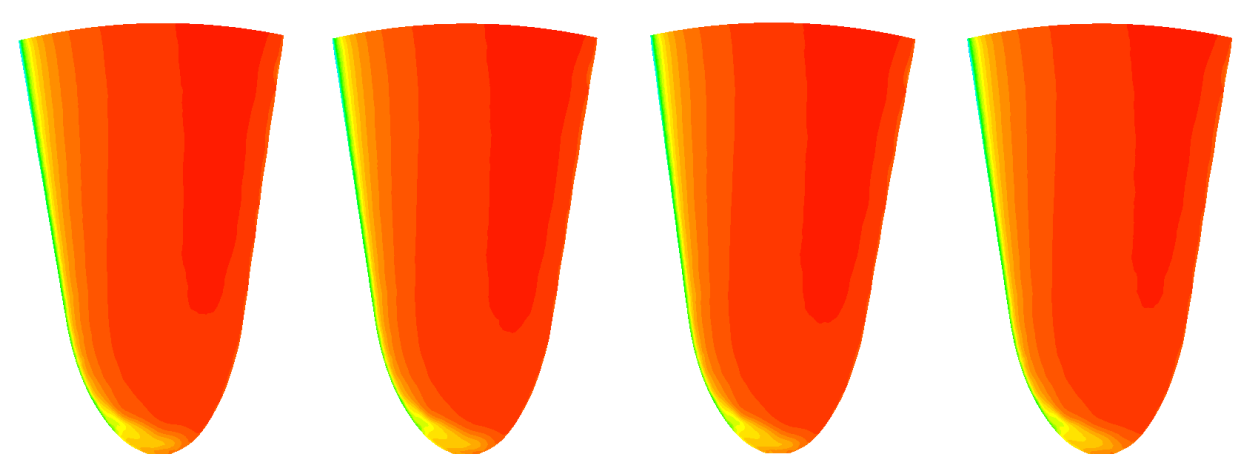

(b) $T S R=1.50$

Figure 20: Pressure coefficient $\left(C_{P r}\right)$ contour representation along the suction edge of the blade at rotation period $\frac{1}{4} T_{\omega}$ (left), $\frac{2}{4} T_{\omega}$ (mid-left), $\frac{3}{4} T_{\omega}$ (mid-right), and $\frac{4}{4} T_{\omega}$ (right) at $U_{\infty}=1.00 \mathrm{~m} / \mathrm{s}$ at low (top) and nominal (bottom) $T S R$

hence, pressure. Precisely at the trailing edge, the suction is substantial enough to engulf the post-separation low-pressure flow towards the wall, increasing the static pressure at this location. This description is consistent at differing radii, yet the extremities decrease proportionally to the radius-position of the blade, primarily due to the comparatively diminished dynamics towards the central axis of the rotor.

In addition to the quantitative measure of static pressure, in an effort to further contemplate the flow dynamics at the blade surfaces, pressure contours along the downstream suction side, illustrated in Figures 20 and 21, were qualitatively attained at low and nominal TSRs at quarterly positions of rotational period $\left(T_{\omega}\right)$ for low and mid free-streams. Regions of stall are elucidated at low rotational velocities, which recede with an increase in TSR; this accompanies well the analysis and speculations put forward as a result of the variation in angle of attack and resultant induced lift. By means of these contours, distinct aspects of 

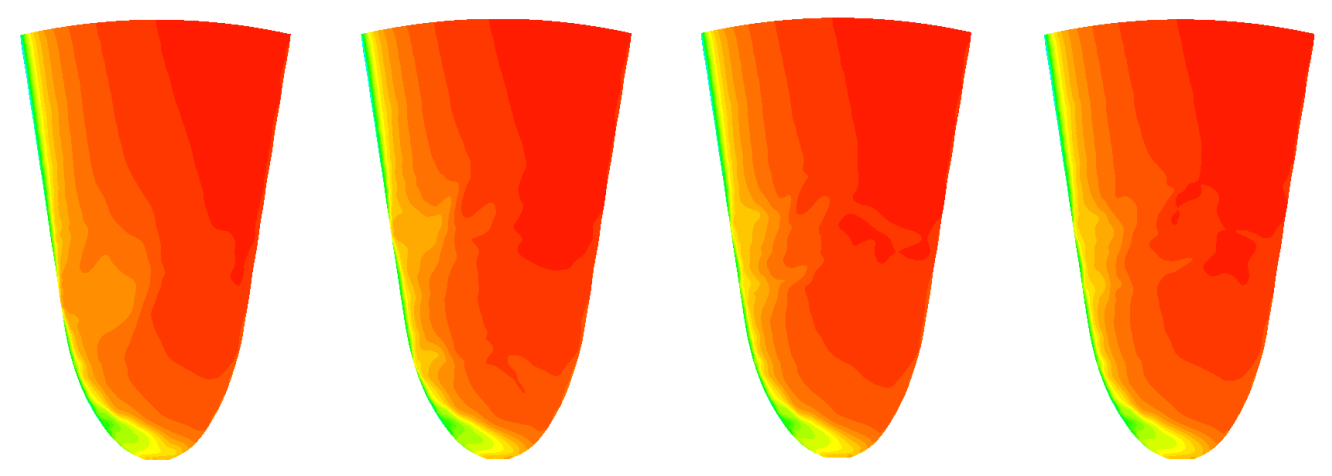

(a) $T S R=1.00$
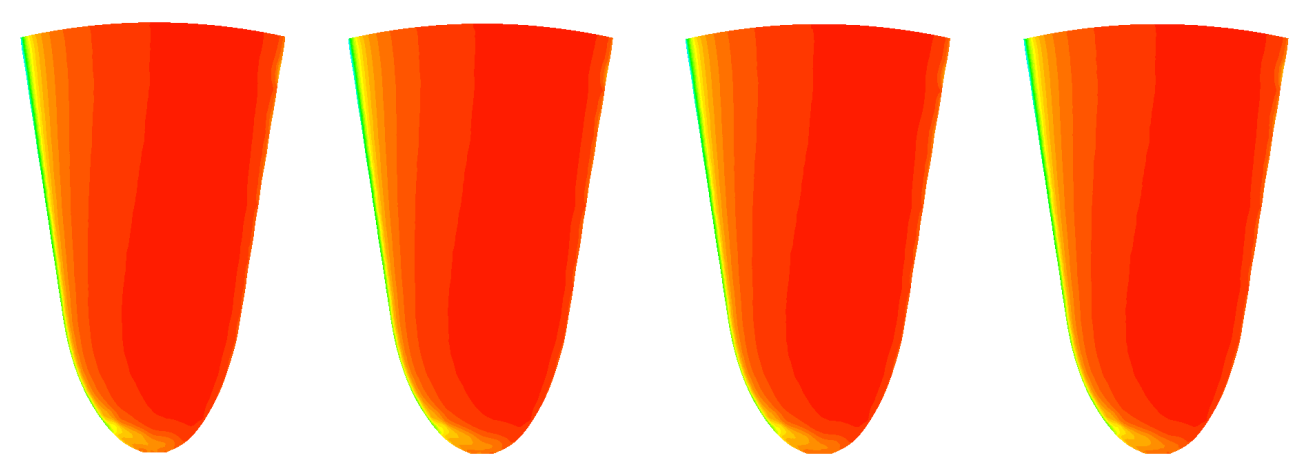

(b) $T S R=1.75$

Figure 21: Pressure coefficient $\left(C_{P r}\right)$ contour representation along the suction edge of the blade at rotation period $\frac{1}{4} T_{\omega}$ (left), $\frac{2}{4} T_{\omega}$ (mid-left), $\frac{3}{4} T_{\omega}$ (mid-right), and $\frac{4}{4} T_{\omega}$ (right) at $U_{\infty}=4.00 \mathrm{~m} / \mathrm{s}$ at low (top) and nominal (bottom) TSR

the flow are established, specifically the spanwise flow acting along the blade, ascertaining the requirement of three-dimensionality when analysing rotating flows.

\subsection{Wake Velocity Profiles}

A potential aspect of the installation of ducts is the constrainment of the downstream wake; an advantage in the effort to limit wake interaction with subsequent rotors within a turbine farm. The axial velocity profiles of the wake of the rotor, from $0.5 D_{r t r}$ to $6 D_{r t r}$ downstream, are uniquely compound as a result of this installation. Illustrated in Figure 22, the dimensionless axial velocity $\left(\bar{u}_{z}^{*}=\frac{\bar{u}_{z}}{U_{\infty}}\right)$ profiles at the distinct free-stream velocities considered are largely identical.

Within the near-wake, at $0.5 D_{r t r}$, the maximum axial wake velocity is identified to be at the centre; a result of the open-centre of the turbine. This velocity surpasses the value of the 


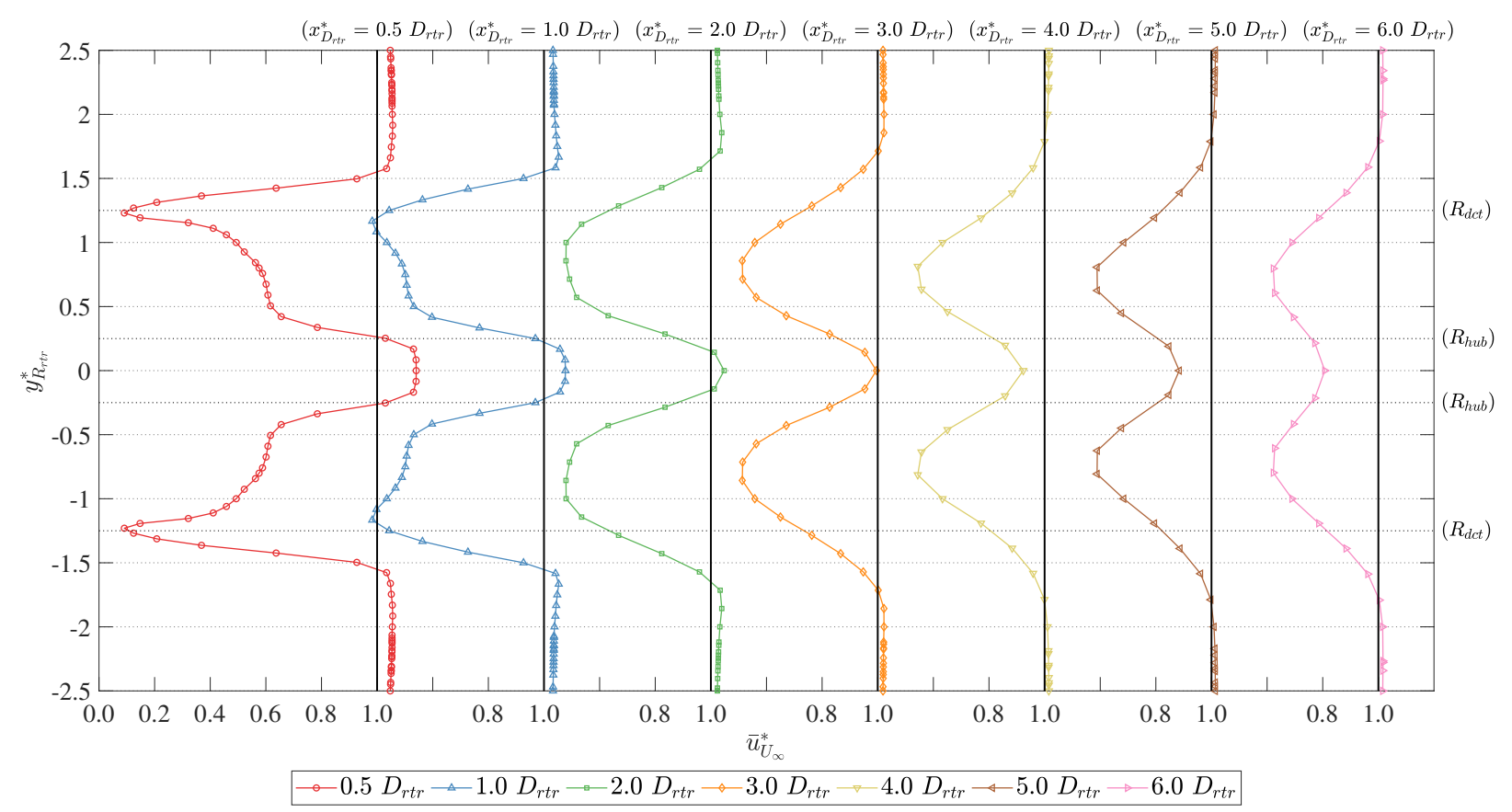

Figure 22: Dimensionless axial velocity $\left(\bar{u}_{z}^{*}\right)$ profiles within the ducted turbine wake at different rotor diameter displacements downstream $-U_{\infty}=4 \mathrm{~m} / \mathrm{s}, T S R=1.75$

free-stream magnitude, acknowledging a phenomenon typically established in flow through an orifice: an induction of flow acceleration. This acceleration comes about in consequence of the substantial static pressure retained within the duct immediately upstream of the rotor, forcing fluid out of the open-centre. This outcome sacrifices torque on the blades, as a degree of fluid at the wall flows in a radial direction towards the orifice at the blade tip, rather than in a tangential orientation along the blades. In continuation, the axial velocity diminishes along the radius of the turbine $\left(y_{R_{r t r}}^{*}=\frac{y}{R_{r t r}}\right)$ due to the presence of the blades and duct, where the point of minima axial velocity is situated at the ridge of the duct. The axial velocity then increases rapidly to a magnitude above the free-stream, an effect from the flow acceleration at the upstream ridge, within a displacement of $1.5 R_{r t r}$ (or $1.2 R_{d c t}$ ) from the central axis of the turbine, portraying the capacity of a duct in restricting the extent of the wake growth. The velocity then decreases gradually to the free-stream magnitude.

As the wake flows further downstream, regions of higher kinetic energy dissipate to regions of lower kinetic energy, hence axial velocity decreases within the region in the vicinity of the orifice to supplement the regions in the vicinity of the duct. The velocity profiles become more rounded, signifying an efficient distribution of energy. Far wake, at $6 D_{r t r}$, the velocity profile has largely regained its energy and becomes more consistent along the turbine radius, albeit portraying its unique aspects, particularly at orifice and duct regions. 


\section{Conclusion}

This study put forward an investigation into the hydrodynamic performance concerning a ducted, high-solidity tidal turbine utilising blade-resolved computational fluid dynamics. The research strived to overcome the limitations of actuator disc and blade-element momentum theory methodologies by implementing a computational fluid dynamic analysis with blade-explicit actuality, coupled with general continuity at both the domain and turbine. This feature allows the aspect of modelling flow three-dimensionality at the turbine, together with induced turbulence effects, in further effort of numerically replicating its true performance. Validated by means of a direct comparison to experimentation in numerically replicating the physics of rotating blades, the numerical outcomes of the main performance factors of the full-scale ducted turbine, portraying a peak power coefficient of 0.34 with a thrust coefficient of 1.00 at a nominal tip-speed ratio of 1.75 , were ascertained to justify the applicability of the full-scale analysis and condone further fluid dynamic analysis, namely the hydrodynamic effects along the duct, mass-flow rate through the duct, vortex shedding within the domain, static pressure along the rotor blades, and velocity profiles within the rotor wake.

In summary of the numerical analysis of a ducted high-solidity tidal turbine, it has been observed that, due to the incorporation of a duct with a high number of blades, and hence blade area, the phenomena induced by the fluid-structure interaction are substantial. In effect of the force equilibrium between the magnitude of the flow interaction at the upstream surfaces of the blades with that at the downstream blade surfaces, the resultant torque is substantial at low TSRs, yet decreases rapidly with an increase in TSR. This short-spanning range in TSR is, hence, a direct attribute of the high-solidity properties of the turbine, and is, therefore, the primal reason of its incapability to acquire high values of mechanical power, albeit the high torque attained. This outcome agrees well with Betz's projections for "medium-speed windmills".

The drag on the rotor was found to decrease significantly with TSR, mainly due to the decreasing static pressure difference at higher rotational to free-stream velocity ratios, in consequence of higher mass-flow rates. The most significant phenomena, however, is induced as a result of the introduction of the duct. A stagnant pressure point is developed along the duct inlet, where fluid upstream of this point is forced out of the inlet to flow along the outer flat surface of the duct. Due to its interaction with the free-stream at the duct ridge, a region of low pressure is created, inducing a re-circulating fluid zone. In effect, along the length of the duct, the static pressure at the flat outer surface of the duct is significantly low, inducing suction. Ring vortices are generated as a result of the re-circulating zone, and are shed at the duct outlet. The vortices commingle with the rotor wake, prompting chaotic flow at low TSRs, which streamlines at higher TSRs.

Having validated the numerical model for rotating bladed systems by means of a direct comparison to experimentation in literature on terms of three distinct parameters, notably power coefficient, thrust coefficient, and velocity profiles in the near and far wake, together with comparison of the full-scale analysis to literature and a secondary numerical methodology, the concluded outcomes are assumed to hold. Albeit, the turbulence model utilised in 
this work has been documented to be oversensitive, and hence, may overestimate physical parameters generated at the wall.

Notwithstanding, this work provides a plausible analysis in evidence to the notion that ducted turbines generate higher power per rotor unit area. This is due to the flow being converged towards the rotor when impacting the inner duct. In elaboration of these claims, the power generated by a ducted rotor would be approximately equal to the power generated by an unducted rotor $\left(P_{r t r_{d c t d}} \approx P_{r t r_{u n d c t d}}\right)$, given that the radius of the duct is equal to the radius of the unducted rotor $\left(R_{d c t}=R_{\text {rtr } r_{\text {undctd }}}\right)$, where the true cut-off area is the region of stagnant pressure on the duct inlet geometry, which is dependent on the cross-sectional formation. Under this prospect, the power per unit rotor area of a ducted rotor would be higher in comparison to the power per unit rotor area of an unducted turbine as the area of the ducted rotor is significantly lesser than that of the unducted rotor $\left(A_{r t r_{d c t d}} \ll A_{r t r_{u n d c t d}}\right)$. Albeit the potential increase in power per rotor unit area, the thrust coefficient, in addition to the complementary phenomena, induced as a result of the implementation of the duct, was found to be physically substantial, and hence, considerably negates any related advantageous aspects of the installation.

In essence, as a result of the induced hydrodynamic Venturi effect, it may be speculated that if the areal annulus in which the duct coincides is lower in both capital and operation cost than the blades present in an identical areal annulus of an unducted turbine, then potentially-sustainable advantages in relation to an implementation of a duct may be present. Albeit this indication, this study solely considers a single geometrical design of a duct within a constant flow in open-water conditions, which brings about a degree of assumptions in comparison to real-world implementations, namely absences of variations in flow direction, differences in inlet turbulence intensities and turbulent length scales, and external inlet shear flow, potentially inducing a distinction between the lift force induced at regions along the duct, developing a resultant lift force, requiring further analysis on its foundational supports.

\section{Acknowledgments}

The research work disclosed in this publication is partially funded by the Endeavour Scholarship Scheme (Malta). Scholarships are part-financed by the European Union - European Social Fund (ESF) - Operational Programme II - Cohesion Policy 2014-2020: "Investing in human capital to create more opportunities and promote the well-being of society".

Results were obtained using ARCHIE-WeSt High Performance Computer (www.archiewest.ac.uk). 


\section{References}

[1] F. O Rourke, F. Boyle, and A. Reynolds, "Tidal energy update 2009," Applied Energy, vol. 87, no. 2, pp. 398-409, 2010.

[2] F. M. White, "Fluid Mechanics," in Fluid Mechanics, ch. 6, pp. 325-426, New York: McGraw Hill, 8th ed., 2015.

[3] S. C. Allsop, Hydrodynamic Modelling for Structural Analysis of Tidal Stream Turbine Blades. PhD thesis, University of Edinburgh, 62018.

[4] S. Allsop, C. Peyrard, P. R. Thies, E. Boulougouris, and G. P. Harrison, "Hydrodynamic analysis of a ducted, open centre tidal stream turbine using blade element momentum theory," Ocean Engineering, vol. 141, pp. 531-542, 92017.

[5] C. Belloni, R. Willden, and G. Houlsby, "An investigation of ducted and open-centre tidal turbines employing CFD-embedded BEM," Renewable Energy, vol. 108, pp. 622-634, 82017.

[6] A. Betz, "Windmills in the light of modern research," tech. rep., National Advisory Committee for Aeronautics, Washington, DC, 81928.

[7] P. Gipe, "WIND-WORKS: Ducted Wind Turbines," 2018.

[8] Bloomberg L.P., "OpenHydro Group Limited: Private Company Information - Bloomberg," 2018.

[9] OpenHydro Group Ltd., "Projects," 2016.

[10] ReNews, "Atlantis-to-decommission-seagen - reNews - Renewable Energy News," 2016.

[11] The Canadian Press, "Cape Sharp Tidal turbine in Bay of Fundy now being monitored remotely," 2018.

[12] E. Benezet, "La grande vague des hydroliennes," 2018.

[13] D. G. Phillips, An investigation on diffuser augmented wind turbine design. PhD thesis, The University of Auckland, 2003.

[14] D. G. Phillips, P. J. Richards, and R. G. J. Flay, "DIFFUSER DEVELOPMENT FOR A DIFFUSER AUGMENTED WIND TURBINE USING COMPUTATIONAL FLUID DYNAMICS," tech. rep., The University of Auckland, Auckland, New Zealand, 2005.

[15] K. D. Visser, "Wind Tamer Turbine Performance Report 1kW-15 kW Performance Predictions," tech. rep., Clarkson University, Potsdam, 2009.

[16] M. O. L. Hansen, N. N. Sørensen, and R. G. J. Flay, "Effect of Placing a Diffuser around a Wind Turbine," Wind Energy, vol. 3, pp. 207-213, 102000.

[17] M. J. Werle and W. M. Presz, "Ducted Wind/Water Turbines and Propellers Revisited," Journal of Propulsion and Power, vol. 24, pp. 1146-1150, 92008.

[18] H. Grassmann, F. Bet, G. Cabras, M. Ceschia, D. Cobai, and C. Delpapa, "A partially static turbinefirst experimental results," Renewable Energy, vol. 28, pp. 1779-1785, 2003.

[19] H. Grassmann, F. Bet, M. Ceschia, and M. Ganis, "On the physics of partially static turbines," Renewable Energy, vol. 29, pp. 491-499, 42004.

[20] Y. Ohya, T. Karasudani, A. Sakurai, K.-i. Abe, and M. Inoue, "Development of a shrouded wind turbine with a flanged diffuser," Journal of Wind Engineering and Industrial Aerodynamics, vol. 96, pp. 524-539, 52008.

[21] C. Munch, M. Vonlanthen, J. Gomes, R. Luquet, P. Guinard, and F. Avellan, "Design and Performance Assessment of a Tidal Ducted Turbine," in 3rd IAHR International Meeting of the Workgroup on Cavitation and Dynamic Problems in Hydraulic Machinery and Systems, (Brno, Czech Republic), 2009.

[22] D. L. Gaden and E. L. Bibeau, "A numerical investigation into the effect of diffusers on the performance of hydro kinetic turbines using a validated momentum source turbine model," Renewable Energy, vol. 35, pp. 1152-1158, 62010.

[23] M. Shives, C. Crawford, and S. Grovue, "A tuned actuator cylinder approach for predicting crossflow turbine performance with wake interaction and channel blockage effects," International Journal of Marine Energy, vol. 18, pp. 30-56, 62017.

[24] U. Ahmed, D. Apsley, I. Afgan, T. Stallard, and P. Stansby, "Fluctuating loads on a tidal turbine due to velocity shear and turbulence: Comparison of CFD with field data," Renewable Energy, vol. 112, pp. 235-246, 112017. 
[25] M. A. Holst, O. G. Dahlhaug, and C. Faudot, "CFD Analysis of Wave-Induced Loads on Tidal Turbine Blades," IEEE Journal of Oceanic Engineering, vol. 40, pp. 506-521, 2015.

[26] T. Setoguchi, N. Shiomi, and K. Kaneko, "Development of two-way diffuser for fluid energy conversion system," Renewable Energy, vol. 29, pp. 1757-1771, 82004.

[27] C. F. Fleming and R. H. Willden, "Analysis of bi-directional ducted tidal turbine performance," International Journal of Marine Energy, vol. 16, pp. 162-173, 2016.

[28] C. S. Belloni, R. H. Willden, and G. T. Houlsby, "A Numerical Analysis of Bidirectional Ducted Tidal Turbines in Yawed Flow," Marine Technology Society Journal, vol. 47, pp. 23-35, 72013.

[29] C. Belloni, Hydrodynamics of Ducted and Open-Centre Tidal Turbines. PhD thesis, Oxford University, UK, 2013.

[30] M. G. Borg, Q. Xiao, A. Incecik, S. Allsop, and C. Peyrard, "Numerical Analysis of a Ducted HighSolidity Tidal Turbine," in OCEANS'18 MTS/IEEE Kobe, (Kobe, Japan), IEEE, 2018.

[31] D. C. Wilcox, Turbulence Modeling for CFD, Third Edition. San Diego: DCW Industries, Inc, third ed., 2006.

[32] P. Mycek, B. Gaurier, G. Germain, G. Pinon, and E. Rivoalen, "Renewable Energy Experimental study of the turbulence intensity effects on marine current turbines behaviour. Part I: One single turbine," Renewable Energy, vol. 66, pp. 729-746, 2014.

[33] S. P. Neill, J. R. Jordan, and S. J. Couch, "Impact of tidal energy converter (TEC) arrays on the dynamics of headland sand banks," Renewable Energy, vol. 37, pp. 387-397, 12012.

[34] A. Bahaj and L. Myers, "Analytical estimates of the energy yield potential from the Alderney Race (Channel Islands) using marine current energy converters," Renewable Energy, vol. 29, pp. 1931-1945, 102004.

[35] ANSYS Inc., FLUENT User's Guide. Lebanon, NH: ANSYS Inc., 5 ed., 1998.

[36] T. Kajishima and K. Taira, Computational Fluid Dynamics: Incompressible Turbulent Flows. Cham: Springer International Publishing, 2017. 
LaTeX Source Files
Click here to download LaTeX Source Files: Numerical Analysis of a Ducted High Solidity Tidal Turbine.zip

LaTeX Source Files
Click here to download LaTeX Source Files: Numerical Analysis of a Ducted High Solidity Tidal Turbine.zip

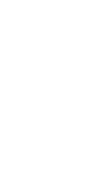
. 西

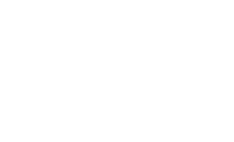
(1) (1) . .

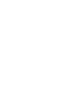

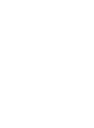
. .

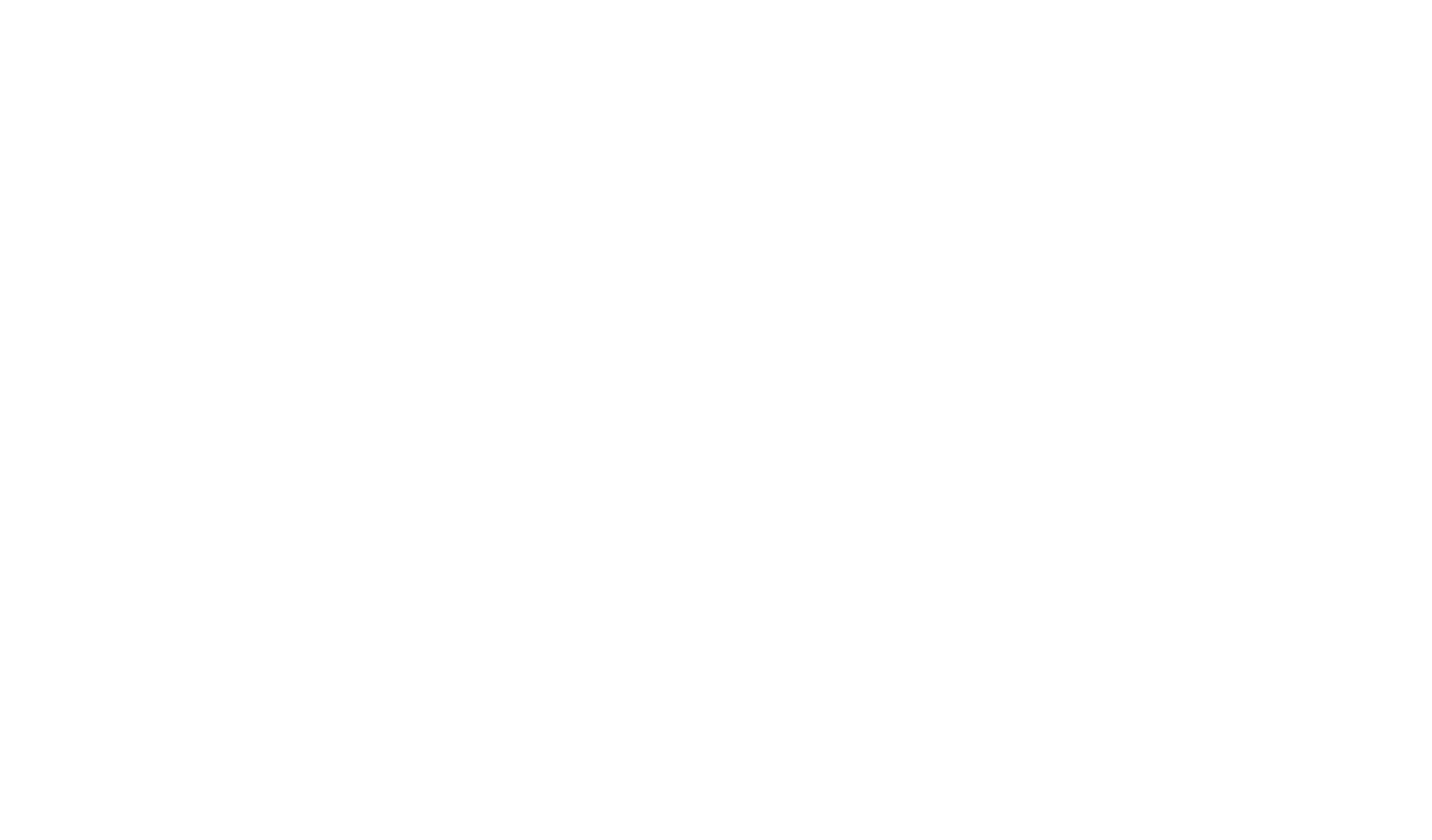
.

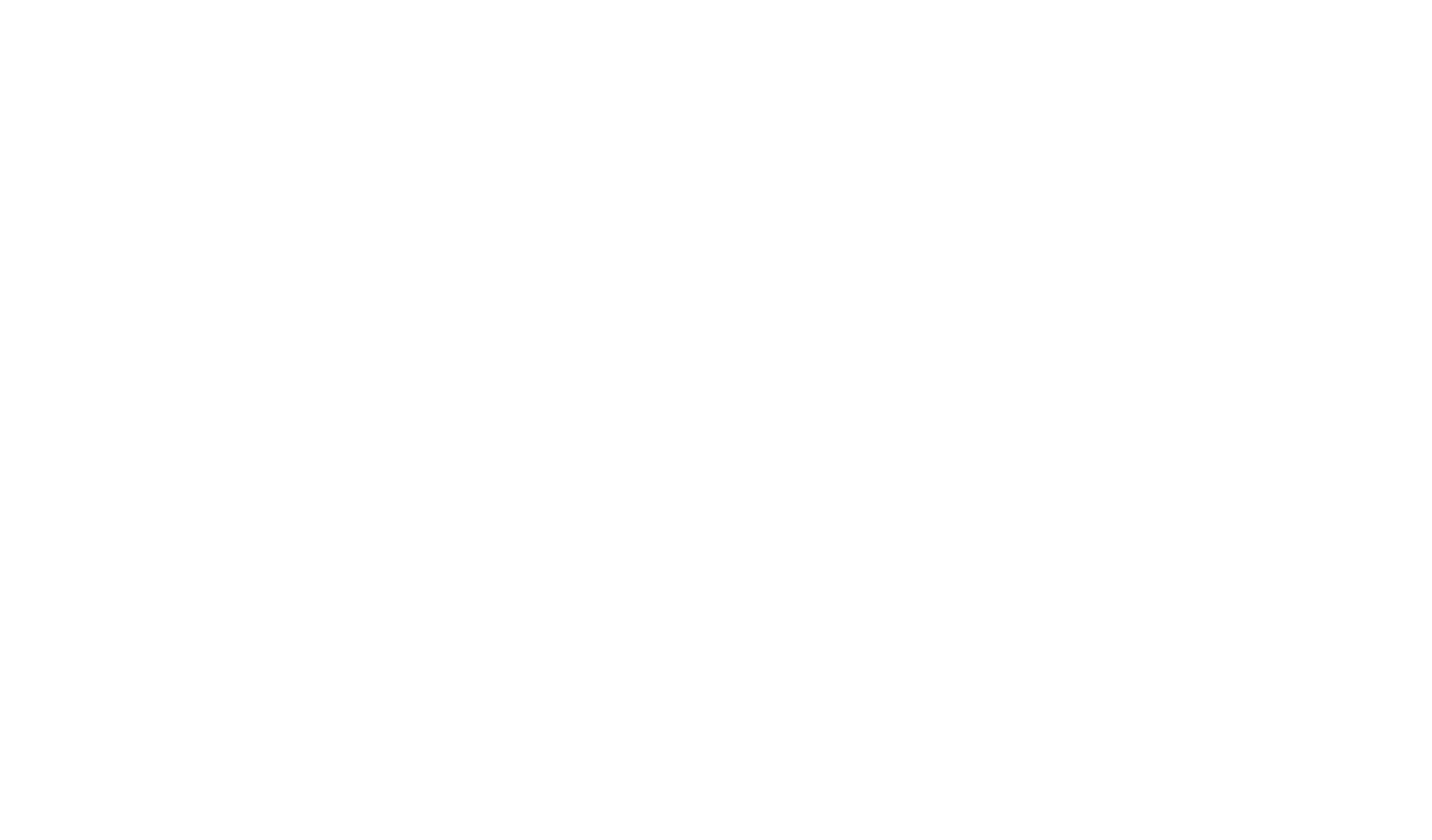
.

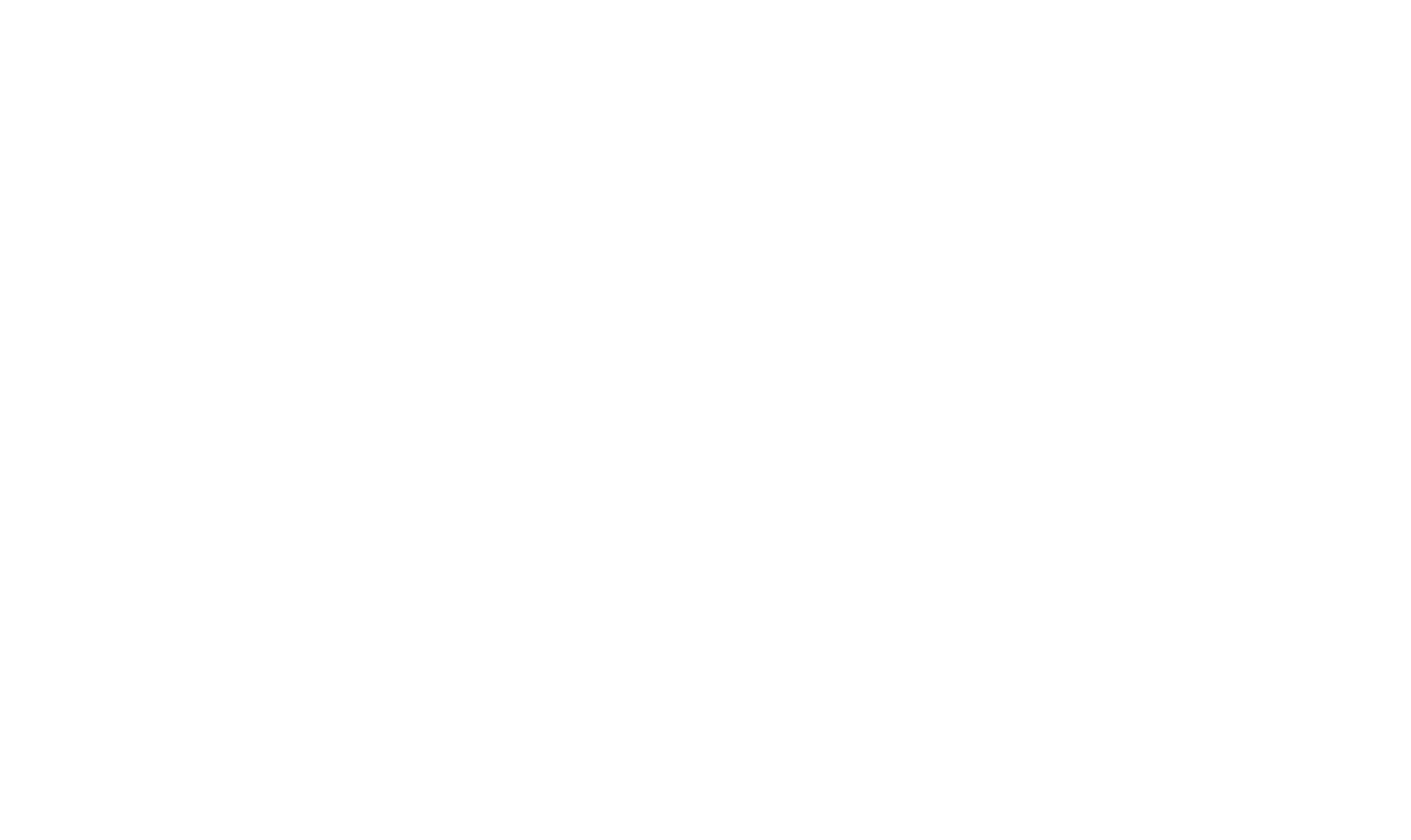
. 政 . .

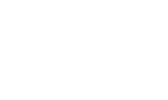


Credit Author Statement

Mitchell Borg - Conceptualisation, methodology, software, validation, formal analysis, investigation, Writing - Original Draft

Qing Xiao - Writing - Review \& Editing, Supervision, resources, project administation

Steven Allsop - Conceptualisation, Writing - Review \& Editing, Resources, formal analysis, investigation

Atilla Incecik - Funding acquisition, resources

Christophe Peyrard - Resources, Supervision 
${ }^{*}$ Declaration of Interest Statement

The research work disclosed in this publication is partially funded by the Endeavour Scholarship Scheme (Malta). Scholarships are part-financed by the European Union - European Social Fund (ESF) - Operational Programme II - Cohesion Policy 2014-2020: 'Investing in human capital to create more opportunities and promote the well-being of society". 